\title{
Strength statistics of single crystals and metallic glasses under small stressed volumes
}

\author{
Yanfei Gao, ${ }^{\mathrm{a}, \mathrm{b}, *}$ Hongbin Bei ${ }^{\mathrm{a}, *}$ \\ ${ }^{a}$ Materials Science and Technology Division, Oak Ridge National Laboratory, Oak Ridge, TN 37831, USA \\ ${ }^{\mathrm{b}}$ Department of Materials Science and Engineering, University of Tennessee, Knoxville, TN 37996, USA
}

\begin{abstract}
It has been well documented that plastic deformation of crystalline and amorphous metals/alloys shows a general trend of "smaller is stronger". The majority of the experimental and modeling studies along this line have been focused on finding and reasoning the scaling slope or exponent in the logarithmic plot of strength versus size. In contrast to this view, here we show that the universal picture should be the thermally activated nucleation mechanisms in small stressed volume, the stochastic behavior as to find the weakest links in intermediate sizes of the stressed volume, and the convolution of these two mechanisms with respect to variables such as indenter radius in nanoindentation pop-in, crystallographic orientation, pre-strain level, sample length as in uniaxial tests, and others. Experiments that cover the entire spectrum of length scales and a unified model that treats both thermal activation and spatial stochasticity have discovered new perspectives in understanding and correlating the strength statistics in a vast of observations in nanoindentation, micro-pillar compression, and fiber/whisker tension tests of single crystals and metallic glasses.
\end{abstract}

Keywords: Strength statistics; Intrinsic thermal-activation mechanism; Extrinsic stochastic mechanism; Universal relationship between strength and sample size

*Corresponding authors: ygao7@utk.edu or gaoy@ornl.gov (Yanfei Gao), beih@ornl.gov (Hongbin Bei) 


\section{Introduction}

Materials research has witnessed significant progresses in both structural and functional applications, such as advanced light-weighted alloys for aerospace and automotive industries, hybrid structures in electronic devices, superalloys and thermal barrier coatings for gas turbines, and micro- and nano-electro-mechanical systems (MEMS/NEMS) that exhibit unprecedented properties, to name a few. To tackle the modern energy challenges, the Basic Energy Sciences Advisory Committee [1] has emphasized a new era of science in which materials functionalities should be designed to specifications and materials microstructures are manipulated at will. Among many lines of efforts in this objective, one challenge that defines the roadmap for structural materials is to push the material performance under extreme environments. For examples, a slight increase of the operation temperature by $50{ }^{\circ} \mathrm{C}$ can dramatically improve the performance of steam turbines, which however demands better creep-resistant alloys [2]; new materials or surface treatments with improved wear resistance may potentially reduce losses in energy conversion [3]; lessons learned from biological materials such as nacre can help design light-weighted biomimetic composites with extreme toughness values [4]; the development of irradiation-tolerant alloys is critical in the next-generation nuclear power plant [5]; the understanding of failure modes and the design layouts to prevent failures in modern electronic devices require fundamental studies of defects and microstructural evolution at extreme thermomechanical-electrical loadings, e.g., GPa-level thermal stress and electromigration-induced cavitation failure [6]; and the lifetime study of thermal barrier coatings is of the foremost importance in gas turbines [7]. Although these examples present the complexity and breadth in pushing the frontiers and extremes of structural materials, one central problem is the study of the inelastic deformation mechanisms across scales and across disciplines.

Plasticity is inherently a multiscale phenomenon, not only because of the interaction among defects, deformation fields, and material microstructures, but also due to the initiation, propagation, and evolution processes of defects that span many scales. For example, dislocation plasticity competes with cleavage at a crack tip in atomistic scales, while crystal plasticity or macroscopic continuum plasticity competes with damage or cavitation processes near crack tips in engineering scales. In the past few decades, the rapid development of small scale mechanical testing and characterization techniques has led to a multitude of studies at the meso- and nanoscales with unprecedented resolutions. One central topic of interest is the dependence of 
macroscopic mechanical properties on one or many length scales. Sometimes we have obvious microstructural length scales such as the Hall-Petch relationship between flow stress and grain size in polycrystals, while oftentimes there is no geometric length scale in the problem of interest such as the variation of flow stress in nanoindentation and bending tests. The use of self-similar pyramidal or conical indenters dictates that there is no geometric length scale, but the measured hardness is found to increase rapidly with the decrease of the indentation depth [8]. One interpretation of such indentation size effects (ISEs) originates from the geometrically necessary dislocations (GNDs) that must be present to accommodate the strain gradients. The GND density is found to scale inversely to the indentation depth, thus leading to additional hardening at small depths [8-10]. However, predictions based on GNDs significantly overestimate the hardness in nanoindentation regime, and also the load-displacement curves in depths $<\sim 100 \mathrm{~nm}$ exhibit discontinuous features that defies any continuum models $[11,12]$.

A recent experimental procedure that excludes the macroscopic strain gradients and thus the GND effects is the micro-pillar tests, in which focus ion beam (FIB) milling is usually employed to make micrometer-sized pillars for uniaxial tests [9,13-15]. These experiments have usually been done with a fixed length-to-diameter ratio, and the relationship between the yield or flow stress and the pillar diameter is of their central interest. The nanoindentation instrument equipped with a flat-ended indenter is typically used for these compression tests, while microtensile tests are restricted to FIB-milled dog-bone-shaped samples in MEMS-type loading apparatus. Extensive reviews have been provided for these measurements (e.g., [9,15-17]). Here we only summarize representative results in Fig. 1(a) for comparisons to our experiments shown later that cover a much wider length spectrum and that conclude differently from the scaling relationship in these literature works.

As summarized in Fig. 1(a) that plots the normal yield or flow stress with respect to the pillar diameter, early works on FIB-milled Ni pillars [13] cover a range of 1-50 $\mu \mathrm{m}$ and $\mathrm{Au}$ pillars [18,19] in a range of $200 \mathrm{~nm}$ to $10 \mu \mathrm{m}$. Further experiments for other FCC single crystals with FIB milling or electroplating [20-26] all fall into a narrow band with a slope between -0.5 and -1.0 in this log-log plot. In contrast to the FCC observations, the micro-pillars tests on BCC single crystals differ rather dramatically. While tests for V [27] resemble those of FCC single crystals, results for Mo [28] are quite different in the slope value. More experimental compilations can be found in Uchic et al. [15] and Greer and de Hosson [17]. 
All these experiments for single crystals in Fig. 1(a) focus on identifying the mechanisms responsible for the scaling slope in the log-log plot (or equivalently, the power-law exponent in the linear plot). Two commonly accepted models include: (i) dislocation starvation hardening, which argues that the dislocations will escape to the free surface more rapidly than they are generated, thus requiring additional stress to activate continuous dislocation nucleations; and (ii) the truncation of spiral dislocation sources, since the dislocation nucleation sources are not points and the geometric size provides a constraint to the activation of these spiral dislocation sources $[9,17,25]$. The first one was developed from the macroscopic strength measurements (e.g., Fig. 1(a)) and the scanning electron microscopy (SEM) images of slip traces. The second one was mainly derived from in situ transmission electron microscopy (TEM) observations that quantitatively determine the evolution of dislocation microstructure [25], as well as from extensive discrete dislocation dynamics (DD or DDD) simulations [29-33]. For example, Benzerga et al. [34,35] used a two-dimensional DD model but with additional short-range constitutive laws for dislocation junction formation and annihilation, dynamic source and obstacle creation, line tensions, and others, which they term as 2.5D DD model. Their simulations show a transition from forest hardening (as commonly seen in classic metal plasticity) to exhaustion hardening (when decreasing initial source density), as shown schematically in Fig. 1(a). Three-dimensional DD simulations by [29,30] found the operation of single junction-stabilized spiral sources, and in the deformation stage between the activation of these sources, deformation could be nearly elastic or controlled by the dislocation-starvation model. Ryu et al. [33] conducted three-dimensional DD simulations using the ParaDiS code (Lawrence Livermore National Laboratory) and also confirmed the contributions from both dislocation starvation and truncation of single arm source.

The present paper is, however, not another review of the scaling relationship as in Fig. 1(a); rather, it extends this commonly accepted view by pushing the experiments and modeling works to a much wider length spectrum, as shown in Figs. 1(b) and 1(c). When using spherical nanoindentation in carefully prepared and polished samples, the load-displacement curves display sudden displacement excursions, oftentimes indicating a burst of dislocation plasticity events. Before the first pop-in, the load-displacement curve can be described by the Hertzian elastic contact theory, so that the maximum resolved shear stress (RSS) at this first pop-in, $\tau_{R S S}^{\max }$, also denoted as the pop-in stress, $\tau_{\text {pop-in }}$, can be used as the yield strength. Note that since these 
tests are conducted on single crystals, it is the resolved shear stress rather than the Mises effective stress that dictates the incipient plasticity. A semi-log plot of the pop-in stress against the indenter radius is presented in Fig. 1(b) for Mo single crystals [36,37]. There are three major differences from Fig. 1(a). First, the pop-in tests at very small stressed volumes (i.e., indenter radius $600 \mathrm{~nm}$ ) give rise to $\tau_{R S S}^{\max }$ at the theoretical strength of $\sim 15.7 \mathrm{GPa}$, agreeing perfectly with density functional theory calculations [38]. There is no size effect in this range of indenter radii. In contrast, the largest measured shear strength is <2 GPa for Mo single crystals (note that a Schmid factor is needed in converting the normal strength to shear strength) in Fig. 1(a). Second, when the indenter radius falls into the range of $\sim 600 \mathrm{~nm}$ to $\sim 50 \mu \mathrm{m}$, the pop-in stress varies dramatically between the theoretical shear strength and the bulk flow stress of about $700 \mathrm{MPa}$. The percentage curves are modeling results that indicate the cumulative probability contours. For example, $90 \%$ curve at a given indenter radius means that $90 \%$ of the indentation pop-in data will fall below this curve. The scatter is too significant to extract any meaningful scaling exponent, which is in sharp contrast to the narrow band of Mo data in Fig. 1(a). Third, the scattering range increases and then decreases as the increase of the indenter radius, and eventually a plateau value of bulk flow stress is reached with negligible scatter. It should be noted that nanoindentation pop-in tests can be easily repeated hundreds of times as shown by the data points in Fig. 1(b), while pillar tests usually present a handful of data points. Consequently, it is anticipated that the size effects should be described by two asymptotes at extremely small and extremely large stressed volumes, together with stochasticity in the intermediate volume.

If one is not convinced by the comparison between Figs. 1(a) and 1(b) since the latter is nanoindentation pop-in tests, we present Mo-alloy pillar tests in Fig. 1(c) [39,40]. These pillars are Mo-10Al-4Ni (in at\%) alloys prepared by directional solidification of NiAl-Mo eutectic alloy [41-43]. Below the eutectic temperature, a eutectic alloy will separate into two phases. If the temperature gradient is one dimensional as in the directional solidification technique, the separated phases will form columnar or lamellar structures. After etching away the NiAl matrix, the Mo-alloy pillars can be used for mechanical tests. The as-grown samples have extremely low pre-existing defect density since only liquid-solid phase transformation is involved, as proved in TEM studies in Sudharshan Phani et al. [44]. As shown in Fig. 1(c), not only the as-grown samples do not show any size dependence, but also the normal yield stress is about 5 times the FIB-milled Mo fibers in Fig. 1(a). This corresponds to the stage of pop-in stress when the 
indenter radius is less than about $600 \mathrm{~nm}$ in Fig. 1(b), i.e., approaching an upper asymptote. Furthermore, the phase-separated eutectic alloys were pre-strained to $4 \%$ and $11 \%$ of thickness reduction, and then Mo-alloy fibers were prepared by etching away the matrix. The $11 \%$ prestrained samples give the bulk flow stress, with some scatter at $300 \mathrm{~nm}$ diameter case. This corresponds to the stage of pop-in stress when the indenter radius is larger than about $200 \mu \mathrm{m}$ in Fig. 1(b), i.e., approaching the bulk strength asymptote. The 4\% pre-strained samples show scattering as the intermediate case in Fig. 1(b). These observations highlight another important variable in small scale mechanical testing, i.e., the initial defect density. If the sample size can be normalized by the initial defect density, it is anticipated that our results in Fig. 1(c) will again exhibit the three-stage curves as in Fig. 1(b). On the other hand, the measurements in Fig. 1(b) can also vary with respect to the change of initial defect density. The dashed curves in Fig. 1(c) schematically indicate the $10 \%$ and $90 \%$ cumulative probability contour curves.

The contrasting behavior between Fig. 1 (a) and (b)/(c) can also be found in small scale mechanical testing of metallic glasses (MG) or amorphous alloys. To differentiate from the metallic glass ribbons, the terminology of bulk metallic glass (BMG) is commonly adopted. Because of the lack of dislocations, grain boundaries, or other microstructural features, our understanding of the plastic deformation behavior in metallic glasses falls behind that of crystalline materials [47-49]. Macroscopically, metallic glasses rarely show any noticeable ductility in tension, and upon yield, the deformation field rapidly localizes into shear bands which subsequently evolve into fracture [50-53]. Microscopically, the initiation of shear band involves the activation of atomistic defects such as free volume or shear transformation zone, which are locations where atomic packing deviates from the ideal glassy structure [47,54-57]. Representative strength measurements from micro-pillar compression tests are complied in Fig. 1(d). As pointed out in a number of small scale mechanical tests [51,58,59], because smaller samples have lower spring stiffness, the strain softening associated with the shear banding behavior does not lead to the snap-in instability, so that the gradual deformation associated with shear band propagation can be recorded. Scaling relationship similar to those shown by the crystalline pillars in Fig. 1(a) has been sought by many prior works. For example, results for $\mathrm{Zr}$ based metallic glass [60] and Al-based metallic glass [61], as plotted in Fig. 1(a), again give a scaling exponent, much higher than -0.5 , on the logarithmic plot of normal yield strength versus the pillar diameter. 
Nanoindentation pop-in experiments on $\mathrm{Zr}_{52.5} \mathrm{Al}_{10} \mathrm{Ti}_{5} \mathrm{Cu}_{17.9} \mathrm{Ni}_{14.6}$ metallic glasses by using a number of spherical indenters [51] demonstrate the same trend as the results of Mo single crystals, as shown in Fig. 1(b). The two asymptotes are found to be very close in these indentation tests. Also the variation of the metallic glass strength in micro-pillar compression tests is much smaller than that of their crystalline counterparts. As a consequence, several recent works begin to show the upper asymptote as those found in Figs. 1(b) and 1(c). As summarized in Fig. 1(d), Kuzmin et al. [62,63] found the "homogeneous" deformation mode in pillar compression tests with small diameters, and the resulting yield strength reaches a plateau value of about 1.8 $\mathrm{GPa}$ for two types of $\mathrm{Zr}$-based metallic glasses $\left(\mathrm{Zr}_{61.8} \mathrm{Cu}_{18} \mathrm{Ni}_{10.2} \mathrm{Al}_{10}\right.$ and $\mathrm{Zr}_{50} \mathrm{Ti}_{16.5} \mathrm{Cu}_{15} \mathrm{Ni}_{18.5}$. However, this strength appears to be too low and the data scatter is rather significant. In nanoindentation pop-in tests of $\mathrm{Zr}_{52.5} \mathrm{Al}_{10} \mathrm{Ti}_{5} \mathrm{Cu}_{17.9} \mathrm{Ni}_{14.6}$ metallic glasses, $\mathrm{Li}$ et al. [57] found an ideal shear strength of about $3.8 \mathrm{GPa}$ (see also the data in Fig. 1(b)), thus suggesting a normal yield strength of $6.6 \mathrm{GPa}$ (or about $E / 10$ with $E$ being the Young's modulus). The shear and normal yield strengths are related by a factor of $\sqrt{3}$ if Mises yield criterion is used. Also we note that many micro-pillars are tapered, so that Liu et al. [64] suggested that the observed mushrooming deformation at the pillar top is actually caused by shear bands that propagate into the interior of the pillar, giving a suspicious homogeneous deformation mode. Wang et al. [61] suggested that the pillar strength should be bounded between the ideal strength of about $0.05 E$ and the flow strength of about $0.02 E$, but their micro-pillar compression data on Al-based metallic glass show little scatter of the strength because of low number of pillars at each diameter, and the strength only reaches an upper bound of $1.8 \mathrm{GPa}$. Results from Jang et al. [60] have similar trends and thus limitations as Wang et al. [61]. The best micro-pillar compression results, which the authors think can be compared to our nanoindentation pop-in tests in BMGs (in Fig. 1(b) and later shown in Fig. 4), are the work by Bharathula and Flores [65] on $\mathrm{Zr}_{58.6} \mathrm{Cu}_{15.6} \mathrm{Ni}_{12.8} \mathrm{Al}_{10.3} \mathrm{Nb}_{2.8}$ samples. They have found significant scattering of the normal yield strength for their pillars of 200-2,000 nm diameters. These results resemble the measurements on $4 \%$ pre-strained samples in Fig. 1(c). It is thus hypothesized that pre-strained crystalline pillars in Fig. 1(c) or metallic glass pillars with potential damages introduced by FIB in Fig. 1(d) by Bharathula and Flores [65] reveal the right portion of the master plots in Fig. 1(b). Our discussions in Sections 2 and 3 will aim to prove this line of argument. 
From the comparisons in Fig. 1, this review paper attempts to establish a new perspective through a master plot that relates the strength (particularly the strength statistics) to the stressed volume size, with respect to a number of controlling parameters such as crystallographic type and orientation, initial defect density, and loading rates. In Section 2, experiments that cover a length spectrum wider than literature work are summarized, including nanoindentation pop-in of several kinds of single crystals, and micro-pillar tests with varying diameters and lengths, and with varying initial defect densities by different pre-strain levels. We particularly address the conditions under which the theoretical strengths are reached, and also the reason that the nanoindentation pop-in and pillar compression tests do not reach the same level of upper asymptotes. In Section 3, a unified model is presented that treats both the thermally activated process near the theoretical strength and the stochastic behavior in the intermediate size of the stressed volume. In Section 4, a number of successful applications of the established master plot are discussed as case studies, including the change of heterogeneous dislocation-nucleation sources by pre-straining, the role of FIB milling, the surface polishing effects, the strain rate effects, the use of pop-in tests as a nanomechanical structural probe in metallic glasses, and comparisons among pillars, fibers, and whiskers. Concluding remarks will be given in Section 5.

\section{Experiments Covering the Entire Scale Spectrum}

\subsection{Nanoindentation pop-in tests}

Instrumented nanoindentation technique has the capability to record the loaddisplacement information easily to the $\mu \mathrm{N}$ and $\mathrm{nm}$ resolutions, so that it has found tremendous applications in the study of small scale mechanical behavior, failure in small devices, and tribological properties $[9,10,66-68]$. One early application that draws great attention in materials research community is the indentation size effects, that is, with the decrease of the pyramidal indentation depth, the hardness increases. Modeling effort based on the geometrically necessary dislocation (GND) can successfully explain this indentation size effects in the micro-indentation regime, but overestimates in the nanoindentation regime [8,10]. Although modified theories using a maximum allowable density or the decrease of the constraining factor (so that the ratio of the actually stressed volume to the nominal contact volume increases) [11,69-71], the loaddisplacement curves in the nanoindentation regime for carefully polished samples rather exhibit a number of displacement bursts, so that the use of hardness as a continuum mechanics quantity is 
not clearly defined. In fact, for spherical or sphere-conical indentations, there exists a transition from elastic Hertzian contact, to displacement excursions (i.e., pop-ins), and to continuum loaddisplacement responses. Here we take a bottom-up viewpoint since the pop-in measurements give rise to the material strength measurements.

Representative load-displacement curves with single or multiple pop-ins are given in Fig. 2 for Mo and Ni single crystals as complied in [72]. Before the first pop-in, the $P$ - $h$ curve can be described by the Hertzian contact theory,

$$
P=\frac{4}{3} E_{r} \sqrt{R h^{3}}
$$

where $E_{r}$ is the reduced (or effective) indentation modulus. For elastically isotropic solids, it is given by

$$
E_{r}=\left[\left(1-v_{i}^{2}\right) / E_{i}+\left(1-v_{s}^{2}\right) / E_{s}\right]^{-1}
$$

where subscripts $i$ and $s$ denote indenter and substrate, respectively, and $E$ and $v$ are the Young's modulus and Poisson's ratio, respectively [73]. For elastically anisotropic solids, analytical forms in Fourier space can be obtained by using the Stroh formalisms or the BarnettLothe integrals, and numerical evaluations must be performed for the final integral representation of the effective indentation modulus [74-84]. The determination of $R$ and $h$ is actually a nontrivial task. For nanoindentation tests typically operated under sub-micron depths, the machine stiffness is considerably larger than the contact stiffness, so that the depth measurement from the piezoelectric actuator is the actual indentation depth. When using spherical indenters with large radii (larger than a few micrometers), the contribution from the machine displacement cannot be neglected. Also the indenter radius provided by the vendors for smaller indenters is found to deviate from SEM-image-based radius calibration. Thus, a calibration method in $\mathrm{Li}$ et al. [85] has been developed to use two materials with known elastic constants to determine both the indenter radius and the machine stiffness (which shows a nonlinear dependence on the indentation depth).

Pop-ins like those presented in Fig. 2 have been found in a large set of experimental works that have been confirmed to arise from dislocation activities, e.g., Au [86], Ni [87-89], Al [70,90], Mo [91], Cr [92], Pt [93-95], Ti [96], Mg [97-99], FCC-structured Fe-15Ni-15Cr and Fe-30Ni [100], BCC-structured Fe-15Cr and Fe-30Cr [100], Fe-3wt\% $\mathrm{Si}$ [101], $\mathrm{CaF}_{2}$ [69], $\mathrm{Al}_{2} \mathrm{O}_{3}$ 
[102], $\mathrm{Cr}_{3} \mathrm{Si}$ [103], $\alpha$-Silicon Nitride [104], GaAs [101], and many others. Surface treatments and polishing conditions are playing important roles in relating the pop-ins to the actual mechanism. For example, pop-ins were found to be caused by fracture, not by dislocation nucleation, in the works by $[105,106]$.

From the pop-in load, we can determine the maximum shear stress that drives the dislocation activity. It has been shown that regardless of the elastic anisotropy, the maximum contact pressure for Hertzian contact is given by

$$
p_{0}=\left(\frac{6 P E_{r}^{2}}{\pi^{3} R^{2}}\right)^{1 / 3} .
$$

For a given $\alpha$-th slip system, we need to search the entire stress field and find out the maximum resolved shear stress, $\max \left\{\tau_{R S S}^{(\alpha)}\left(x_{i}\right)\right\}$. The comparison to the contact pressure in Eq. (3) defines the indentation Schmid factor of this slip system,

$$
S^{(\alpha)}=\frac{1}{p_{0}} \max _{x_{i}}\left\{\tau_{R S S}^{(\alpha)}\left(x_{i}\right)\right\} .
$$

Obviously, one has to search all the slip systems, and the firstly activated slip system corresponds to the largest indentation Schmid factor,

$$
S=\max _{\alpha}\left(S^{(\alpha)}\right) .
$$

For examples, calculations of the indentation $\mathrm{Schmid}$ factor for $\mathrm{Ni}, \mathrm{NiAl}, \mathrm{Ti}$, and $\mathrm{Mg}$ can be found in $[81,96,98]$.

From the above equations, the relationship between the pop-in load, $P_{p o p-i n}$, and the popin stress, $\tau_{p o p-i n}$, is given by

$$
P_{p o p-i n}=\left(\frac{\tau_{p o p-i n}}{S}\right)^{3} \frac{\pi^{3} R^{2}}{6 E_{r}^{2}},
$$

and the corresponding contact radius is

$$
a_{p o p-i n}=\frac{\pi R \tau_{p o p-i n}}{2 E_{r} S} .
$$

In contrast to the tedious sample preparations in micro-pillar compression tests in Fig. 1(a), nanoindentation pop-in tests can easily cover a wide spectrum of length scales. In Figs. 3 and 4, we will show three means along this line of study. First, the two asymptotes and the 
transition in between can be probed by using a number of spherical indenters in Figs. 1(b) and 3(a). In the Mo <001> pop-in tests in Fig. 3(a), most of the data points are around the theoretical shear strength for indenter radius less than about one micrometer. In these cases, the stressed volume size, according to Eq. (7), is about several tens of nanometers, which is too small to find any pre-existing defects that might activate heterogeneous dislocation nucleation mechanisms. When the indenter radius falls in the range of $1 \sim 10 \mu \mathrm{m}$, the slope of the cumulative probability curves becomes significantly smaller than the ones near the theoretical strength. The corresponding plot in Fig. 1(b) shows the data distribution between the two asymptotes. For larger indenters, the bulk strength asymptote is approached and the cumulative probability curves are again steep. Details of the modeling curves in these two plots will be given in Section 3. Second, we can rotate the surface orientation of a single crystal that shows a strong dependence of $P_{p o p-i n}$ and $a_{p o p-i n}$ on the indentation direction in Fig. 3(b). NiAl single crystal with various orientations were tested using a spherical indenter with $R=580 \mathrm{~nm}$ [81]. In Eqs. (6) and (7), both $E_{r}$ and $S$ vary significantly with respect to the surface orientation since the degree of elastic anisotropy is quite significant for $\mathrm{NiAl}$. If we use the theoretical shear strength, $\tau_{t h}$, for the popin stress, $\tau_{p o p-i n}$, in Eq. (6), the corresponding pop-in loads will obey $P_{p o p-i n}^{\langle 100\rangle}: P_{p o p-i n}^{\langle 110\rangle}: P_{p o p-i n}^{\langle 111\rangle}$ $\approx 1: \frac{1}{2}: \frac{1}{3}$, which is confirmed by the measured pop-in loads at cumulative probability $>90 \%$. For surface orientations close to $\langle 100\rangle$, the cumulative probability curves show long tails, similar to the intermediate case in Fig. 1(b) and Fig. 3(a). The corresponding contact sizes from Eq. (7) are much larger than the rest of orientations, i.e., giving large stressed volume size. These observations suggest a convolution of homogeneous and heterogeneous dislocation nucleation mechanisms. For orientations close to $\langle 111\rangle$, the cumulative probability curves are similar to the curves with $R$ less than about one micron in Fig. 3(a), since the corresponding stressed volume size is quite small.

The third means to cover a wide range of nanoindentation pop-in behavior is by changing the material defect density. That is, an ideal sample without any defect will approach the theoretical strength asymptote, while samples with high defect densities will approach the bulk asymptote. Example given here in Fig. 4 is the pop-in measurements for a Zr-based BMG with various annealing conditions. Metallic glasses are prepared by rapid quenching, so that it is anticipated that various types of non-ideal atomic packing structures exist in the as-cast samples. 
Annealing below the glass transition temperature with various annealing time and annealing temperature gives various degrees of the above-discussed "structural heterogeneity". For all the tests in Fig. 4, annealing at $300{ }^{\circ} \mathrm{C}$ for a week gives the Heaviside-type cumulative probability curve, corresponding to the intrinsic thermal activation mechanism near the theoretical shear strength, being about $3.8 \mathrm{GPa}$ here. Take Fig. 4(d) as an example, and we can see that the transition between as-cast and annealed samples is exactly the same as that in Fig. 3(a), i.e., the transition from the bulk asymptote to the theoretical strength asymptote. We also note the effects of indenter radius in these tests. The smallest indenter, being $0.4 \mu \mathrm{m}$, only leads to results that cover the theoretical strength asymptote and the intermediate state (similar to the results in the range of $R=0.1 \sim 5 \mu \mathrm{m}$ in Figs. 1(b) and 3(a)). A more complete set of data for as-cast sample with a number of indenter radii have been provided in Fig. 1(b). In summary, nanoindentation pop-in tests can readily cover the entire spectrum of strength statistics, i.e., the stochastic nature of the transition between the two asymptotes, by the three means explained in Figs. 3 and 4. It is thus suggested that great care should be placed on the interpretation of nanoindentation pop-in tests in literature work, since a small data set cannot conclude the dominant mechanisms.

\subsection{Strength measurements in micro-pillar compression and fiber tension tests}

For Mo single crystals, it is noted that the nanoindentation pop-in tests give an upper asymptote of shear strength to about $16 \mathrm{GPa}$ in Figs. 1(b) and 3(a), the micro-pillar compression tests using directionally solidified Mo alloy fibers give an upper bound of normal strength to about $10 \mathrm{GPa}$ in Fig. 1(c), and the FIB-milled micro-pillar compression tests in Fig. 1(a) give normal strength values at most to a few GPa. Our systematic experimental studies in Fig. 5 [91] give a mechanistic explanation for the difference among these upper-bound strength values.

For nanoindentation pop-in tests using very small spherical indenters, it is found that the pop-in stress corresponds to the theoretical shear strength. In Fig. 5(a), the corresponding value is about $15.7 \mathrm{GPa}$ for Mo-3Nb single crystal, which agrees perfectly with the first principles calculations [38]. Similar observations have been routinely confirmed in many other single crystals, as well by atomistic simulations [107-110]. Also the strength statistics at the asymptote arises from thermal activation, as will be explained in details in Section 3.1. Micro-pillar compression tests in Fig. 5(a) gives a normal strength of $9.8 \mathrm{GPa}$, or shear strength of $4.6 \mathrm{GPa}$ which is about $1 / 3$ of the theoretical shear strength. Bei et al. [91] argued that the difference arises from surface nucleation of a half dislocation loop or a quarter dislocation loop, because the 
interaction between the incomplete dislocation loops with the free surface reduces the dislocation self-energy, thus making surface dislocation nucleation easier to take place. Further evidences can be seen from a number of atomistic simulations that show surface nucleation in metal wires or pillars [111-114], and from experimental work on the surface nucleation of dislocations in $\mathrm{Au}$ nanoparticles [115] and in Pd nanowhiskers [116]. To investigate the alloying effect since Fig. 5(a) is for Mo-3Nb and Fig. 5(b) is for Mo-10Al-4Ni, we performed nanoindentation pop-in test on top of the Mo-10Al-4Ni pillar in Fig. 5(c), which again gives a pop-in stress very close to the theoretical shear strength.

From the above experiments, it can be concluded that (i) the necessary condition to reach theoretical strength is a sufficiently small stressed volume so that there is no pre-existing defect, (ii) if the stressed volume involves free surface, then surface nucleation will reduce the strength, which is however still much higher than the strength measured in samples with pre-existing defects, (iii) pillar/fiber/wire tests unavoidably involve free surface, so their upper-bound value is a fraction of the theoretical strength, and (iv) indentation tests restrict the stressed volume inside the bulk, thus eliminating the surface effects. An additional experimental evidence to support the last statement is a recent compression test of sub-100 nm Si nano-cubes that reaches the theoretical strength [117], in which the maximum stress is located inside the sample as in the indentation experiment.

We now address two primary reasons that explain why micro-pillar compression tests in Fig. 1(a) do not reach high strengths. The first one is that there are pre-existing defects, as illustrated by the tests of pre-strained pillars in Fig. 6 [40]. The second one is the fiber length, or equivalently the stressed volume size, as illustrated by the fiber tension tests in Fig. 7 [45].

For directionally solidified Mo alloy fibers, the as-grown samples have virtually no defect, so that the micro-pillar compression tests reach the upper asymptote of about $10 \mathrm{GPa}$, as shown in Fig. 6(a). For the tests in Figs. 6(b) and 6(c), the Mo-NiAl composite is first prestrained, and then the NiAl matrix is etched away. Noticeable scatter emerge in the $4 \%$ prestrained sample with edge width of about 1,200 nm in Fig. 6(b), and significant scatter can be found in the thinner pillars in Fig. 6(c). The deformed shapes in the insets show the typical slip planes or slip bands. For the $11 \%$ pre-strained samples, the scatter is significantly reduced and the measured strength approaches the bulk strength of about $1 \mathrm{GPa}$ as given in Fig. 6(d). The defect structures before and after the tests have been confirmed by extensive synchrotron x-ray 
diffraction measurements (using the Advanced Photon Source, Argonne, IL by Barabash et al. [118-121] and Bei et al. [122]) and the Swiss Light Source by Zimmermann et al. [123-125], neutron diffraction characterization [126], and TEM investigations [44,45,127,128]. Results in Fig. 6 suggest that the pre-existing defects provide heterogeneous dislocation nucleation sources that lead to significant scatter of the strength measurements, so that seeking for the scaling relationship as in Fig. 1(a) is rather inappropriate.

Using the same directionally solidified Mo alloy fibers, Chisholm et al. [45] conducted in situ TEM studies of tensile tests on as-grown and pre-strained samples. In Fig. 7(a), although the dislocation density is very low, the stressed volume is much larger than that in Fig. 6(a) since the fiber length here is about $5 \mu \mathrm{m}$, so that the measured strength of about $7 \mathrm{GPa}$ still does not reach $10 \mathrm{GPa}$ in the short pillar compression test. This is not tension/compression asymmetry; rather it is the increase of the stress volume size that shifts the strength from the upper asymptote to the intermediate stage. For Mo alloy fibers with more pre-existing defects, Fig. 7(b) shows a much lower strength at about $1.7 \mathrm{GPa}$, which is consistent with the pre-strained samples in Fig. 6 and the FIB-milled samples to be discussed in Figs. 11 and 12. After the plastic yield, dislocation starvation and exhaustion hardening have been found to be responsible for the increase of the flow stress. In situ SEM tests of as-grown Mo alloy fibers under tension by Loya et al. [46] have found similar strengths scatter, as given in Fig. 1(c).

\subsection{Strength statistics - a new perspective on the master plot}

From the nanoindentation pop-in experiments (varying indenter radius in Fig. 1(a) and Fig. 3(a), varying surface crystallographic orientation in Fig. 3(b), and varying initial defect density in Fig. 4), micro-pillar compression tests of directionally solidified Mo alloy fibers (varying pre-strain in Fig. 1(c) and Fig. 6), and fiber tension tests in Fig. 1(c) and Fig. 7, we construct the master plot in Fig. 8, which relates the measured strength and the normalized stressed volume size. The three stages are presented below.

(1) Upper asymptote: When the stressed volume is small (e.g., by using a small spherical indenter or by a short pillar/fiber), or the defect density is low (e.g., by directional solidification that does not involve FIB damage, or by annealing), or the Schmid factor is high (e.g., by indenting a "soft" direction with a high indentation Schmid factor so that the stressed volume becomes small as predicted in Eq. (7)), we will reach the theoretical shear stress, $\tau_{t h}$, if the stressed volume does not contain free surfaces. The theoretical stress falls in the range of 
$G / 5 \sim G / 30$ with $G$ being the shear modulus on the activated slip system. If the stressed volume contains free surfaces or edges, surface nucleation of dislocations will reduce this upper-bound value from $\sigma_{t h}$ to about $\sigma_{t h} / 3$. The measured strength shows a certain degree of scatter, as high as $10 \sim 20 \%$ of $\tau_{t h}$, arising from thermally activated, mechanically assisted process for homogeneous dislocation nucleation. This will be modeled in Section 3.1.

(2) Lower asymptote: For a very large stressed volume, or very large defect density, or very low Schmid factor, the measured strength reaches the bulk flow stress. Nanoindentation tests near this asymptote may or may not observe clear pop-ins. The bulk strength depends on the initial defect density and many other factors, and thus can be tuned by mechanisms such as strain hardening and strain rate sensitivity. However, it should be noted that this lower bound value is usually in the range of $G / 100 \sim G / 1000$, i.e., one or two orders of magnitude smaller than the upper bound value. Therefore, any change of this bulk strength cannot really be seen noticeably on this plot in Fig. 8. One example along this line will be given in Figs. 14 and 15.

(3) Intermediate stage with stochastic behavior: In between the above two asymptotes, we have an intermediate stage in which the stochastic behavior is observed. In Fig. 8, we schematically show the $10 \%$ and $90 \%$ cumulative probability contours, which roughly bound the measured strength values. In nanoindentation pop-in tests using a number of spherical indenters as in Figs. 1(b) and 3(a), this intermediate stage is very clear to determine. In this stage, the strength scatter arises from the random distribution of pre-existing defects. It is the probability of finding a heterogeneous dislocation nucleation source in pop-in tests, or the probability of finding a weakest link (e.g., a Frank-Read source with low strength) in the fiber/pillar tests that dictate these scatters in this stage. This weakest link view is quite universal in brittle materials such as ceramics and rocks, but its importance in ductile materials emerges only in small scales, as reviewed in Section 2.2 and in many other experimental works [65,129,130]. A stochastic model along this line will be presented in Section 3.2. Now revisit the experiments presented in Section 2.2. For the micro-pillar compression tests in Fig. 6, the strength measurements in Fig. 6(a) for as-grown samples fall into the range of (A)-(B) in Fig. 8; data in Figs. 6(b) and 6(c) fall into the range of (C)-(D) in Fig. 8; and the 11\% pre-strained data in Fig. 6(d) are close to state (D) in Fig. 8. For the fiber tension tests in Fig. 1(c) and Fig. 7, although the defect density is low, the stressed volume size is so large that these strength data are actually near the state $(\mathrm{C})$ in Fig. 8. 


\section{Convolution between Thermal Activation Process and Weakest Link Model}

Following the master plot in Fig. 8, our theoretical model will first treat thermal activation and stochastic behavior separately, and then a unified model is presented to cover the entire spectrum of length scales. The following review mainly follows our recent publications $[36,37,81,91]$.

\subsection{Thermally activated dislocation nucleation process}

There is a vast of thermally activated, mechanically assisted processes in materials science. When the applied load is lower than, but not too far from, the athermal limit for the deformation process of interest, an energy barrier exists which can be overcome by thermal energy. The classic model along this line involves an Arrhenius relationship for the nucleation rate and a first-order transition state theory. Earliest applications in nanoindentation pop-in have been found in Schuh et al. [93-95] and Ngan et al. [114,131]. The rate of dislocation nucleation on the $\alpha$-th slip system is given by

$$
\dot{n}^{(\alpha)}=\dot{n}_{0} \exp \left[-\frac{\Delta \Pi^{(\alpha)}\left(\tau_{R S S}^{(\alpha)}\right)}{k_{B} T}\right],
$$

where $\dot{n}_{0}$ is an attempt frequency per volume, $k_{B}$ is the Boltzmann constant, and $T$ is the absolute temperature. The activation energy for homogenous dislocation nucleation, $\Delta \Pi$, is a function of the applied shear stress, $\tau_{\text {appl }}$ or $\tau_{R S S}$. Denote $q_{\text {homo }}^{(\alpha)}$ as the survivability, that is, the probability for no pop-in to take place, where the subscript indicates the homogeneous dislocation nucleation mechanism. The first-order transition state theory gives

$$
-\frac{\dot{q}_{\text {homo }}^{(\alpha)}}{q_{\text {homo }}^{(\alpha)}}=\dot{n}^{(\alpha)} V,
$$

where $V$ is the stressed volume. Since the indentation load and resolved shear stress are related through Eq. (6), integrating Eq. (9) gives

$$
\ln q_{\mathrm{homo}}^{(\alpha)}=-\dot{n}_{0} V \int_{0}^{P_{\text {pop } \text {-in }}} \exp \left[-\frac{\Delta \Pi^{(\alpha)}\left(\tau_{R S S}^{(\alpha)}(P)\right)}{k_{B} T}\right] \frac{d P}{\dot{P}} .
$$

The cumulative pop-in probability is given by 


$$
f_{\text {homo }}=1-\prod_{\alpha} q_{\text {homo }}^{(\alpha)} .
$$

The central task in the above model is the representation of $\Delta \Pi\left(\tau_{\text {appl }}\right)$. Schuh and Lund [93] adopted the Taylor expansion,

$$
\Delta \Pi\left(\tau_{\text {appl }}\right)=\Pi_{0}-\tau_{\text {appl }} \Omega,
$$

where $\Omega$ is the activation volume. Therefore, when fitting Eq. (11) to the measured cumulative probability curves, the fitting parameters include $\dot{n}_{0}$ and $\Omega$.

Generally, in thermally activated processes, when the applied stress is lower than the athermal critical value, there are two solutions, one being the stable solution as obtained from gradual loading from zero to the current stress, and the other being the metastable solution. The transition between these two states experiences a saddle point configuration which has a higher energy than the stable solution, and the energy difference between the saddle point and stable configurations is the activation energy. Such a general view has be adopted for various types of dislocation models, including the Volterra dislocation analysis [91,132-134], the Peierls dislocation model [135-140], or molecular dynamics [131,142]. The saddle point configuration can be guessed in simple cases, e.g., a circular dislocation loop in homogeneous dislocation nucleation, or a half loop from a crack tip or surface steps. But a general way of determining the saddle point configuration requires algorithms to determine the minimum energy pathway, such as the nudged elastic band (NEB) method. Examples of NEB applications can be found in molecular simulations [113,141] and first principles calculations [143]. After compiling a number of available calculations, $\mathrm{Li}$ et al. [81] suggested the following representation for homogeneous dislocation nucleation,

$$
\Delta \Pi=A\left(1-\frac{\tau_{\text {appl }}}{\tau_{\text {th }}}\right)^{n},
$$

where the exponent $n$ is in the range of 1.5 4.5, and the pre-factor $A$ is in the range of (5 15) $G b^{3}$ with $b$ being the magnitude of Burgers vector. To this end, when fitting to experimental measurements, the fitting parameters include $\dot{n}_{0}$ and $\tau_{t h}$.

Although successes from the above model has been demonstrated in a number of works [81,91,93-95,144], a caveat should be noted that the thermal activation analysis works strictly in state (A) in Fig. 8. If the stochastic behavior contributes to the cumulative probability, i.e., near 
state (B), the use of the thermal activation model in Eq. (11) will give incorrect predictions. Using the pop-in results of $\mathrm{NiAl}<207>$ indentation in Fig. 2(b) as an example, it can be seen that the cumulative probability has a long tail at low pop-in load. Converting the cumulative probability to probability, $p=d f / d P_{p o p-i n}$, (or histograms) in Fig. 9(a), two Gaussian peaks are observed. Only the one at high pop-in load corresponds to the thermal activation process, while the left one corresponds to a heterogeneous process to be discussed in Section 3.2. Blindly fitting to the upper portion of the cumulative probability curve gives the solid line, while only fitting to the right Gaussian peak gives the dashed curve in Fig. 9(b). Should we have used the activation volume method in Eq. (12), the incorrect fitting to the upper portion of the cumulative probability curves would incorrectly give a higher activation volume, while the correct fitting to the right Gaussian peaks will find the same activation volume for all crystallographic orientation. We also note that fitting curves for <101> indentation are almost indistinguishable, because the Gaussian peak at low load has a low height and also is buried in the Gaussian peak at high load, similar to the superposition of diffraction peaks in x-ray and neutron experiments $[2,145]$.

The stochastic behavior described in Figs. 8 and 9 is from the statistical distribution of pre-existing defects. Ngan and his group [114,131] have revealed another origin of stochasticity by the statistical profiles of atomic properties such velocities and energies. A weak size effect is shown from their molecular dynamics simulations for homogeneous dislocation nucleation and surface nucleations, which can be rationalized by this atomistic stochasticity. Moreover, their weak size effect for surface nucleation has been confirmed by experimental studies in dislocation-free $\mathrm{Ni3Al}$ nanocubes [146].

\subsection{Unifying the spatial stochasticity and thermal activation}

The range of (B)-(C) in Fig. 8 is dominated by the spatial stochasticity, i.e., the probability of finding a pre-existing source for heterogeneous dislocation nucleation, such as the Frank-Read source, single spiral arm source, or other types of instabilities [36,44,147-150]. It is not our intention to provide a detailed analysis of these events, since many DDD and molecular simulations have been devoted along these lines. Rather, we note that the most important feature is the spacing of these heterogeneous sources. Following Morris et al. [148], we simply assume that the pop-in will occur when a pre-existing defect (or heterogeneous source) can be found in the stressed volume, $V_{d}$, wherein the applied shear stress is larger than its strength, $\tau_{\text {defect }}$. The 
stochasticity is modeled by a Poisson distribution, that is, the surviving probability, $q_{\text {hetero }}$, that there exists no defect in $V_{d}$ is given by

$$
q_{\text {hetero }}\left(\rho_{\text {defect }}, V_{d}\right)=\exp \left(-\rho_{\text {defect }} V_{d}\right)
$$

where $\rho_{\text {defect }}$ is the pre-existing defect density. Because point sources are assumed, the unit of $\rho_{\text {defect }}$ is $[\text { length }]^{-3}$, and the spacing is given by $\rho_{\text {defect }}^{-1 / 3}$. Apparently, the larger the stressed volume, the more probable it is to find a pre-existing defect. If two volumes, $V_{d, 1}$ and $V_{d, 2}$, are combined, elementary statistics gives the total survivability as $q_{\text {hetero }}\left(V_{d, 1}+V_{d, 2}\right)=q_{\text {hetero }}\left(V_{d, 1}\right) q_{\text {hetero }}\left(V_{d, 2}\right)$ $=\exp \left[-\rho_{\text {defect }}\left(V_{d, 1}+V_{d, 2}\right)\right]$, suggesting that the Poisson's distribution is equivalent to the Weibull analysis.

When using the above equation for nanoindentation pop-in, we note that $V_{d}$ varies with respect to the applied load, and can be represented by a dimensionless function

$$
\frac{V_{d}^{(\alpha)}}{a^{3}}=\hat{V}_{d}^{(\alpha)}\left(\frac{S^{(\alpha)} p_{0}}{\tau_{\text {defect }}}, \frac{c_{I J}}{c_{11}} \text {, indentation direction }\right)
$$

where $c_{I J}$ are elastic constants in Voigt contracted notation. Evaluation of the above equation involves a tedious, but straightforward, anisotropic elastic contact analysis [36]. Therefore, the cumulative probability for defect assisted pop-in is

$$
f_{\text {hetero }}=1-\prod_{\alpha} q_{\text {hetero }}^{(\alpha)}=1-\exp \left(-\rho_{\text {defect }} \sum_{\alpha} V_{d}^{(\alpha)}\right) .
$$

The thermally activated, homogeneous dislocation nucleation model in Eq. (11) and the defect assisted model in Eq. (16) are independent statistical processes. Thus the total cumulative probability for pop-in to occur is given by

$$
\begin{aligned}
& f_{\text {total }}=1-q_{\text {total }}=1-\left(\prod_{\alpha} q_{\text {hetero }}^{(\alpha)}\right) \times\left(\prod_{\alpha} q_{\text {homo }}^{(\alpha)}\right) \\
& =1-\exp \left\{-\rho_{\text {defect }} \sum_{\alpha} V_{d}^{(\alpha)}-\dot{n}_{0} \sum_{\alpha} \int_{0}^{P_{\text {pop-in }}} \iiint_{\Omega} \exp \left[-\frac{\Delta \Pi^{(\alpha)}\left(\tau_{R S S}^{(\alpha)}\left(x_{i}\right)\right)}{k_{B} T}\right] d \Omega \frac{d P}{\dot{P}}\right\}
\end{aligned}
$$

The fitting parameters include the attempt frequency $\dot{n}_{0}$ and theoretical strength $\tau_{t h}$ (or the activation volume) for the thermal activation part, which should not vary with respect to the 
stressed volume size, and the defect density $\rho_{\text {defect }}$ and defect strength $\tau_{\text {defect }}$ for the heterogeneous, stochastic process. As shown in Fig. 10(a), as the increase of the stressed volume size or the increase of the defect density, we observe a transition from homogeneous nucleation dominated plasticity, to the intermediate stochastic plasticity, and to the conventional plasticity. Using the unified model in Eq. (17), we have successfully explained the indenter radius effect in Figs. 1(b) and 3(a) for Mo single crystals, the indentation orientation effect in Fig. 3(b) for NiAl single crystals, and the annealing effects in Fig. 4 for a Zr-based BMG. In the first two examples, since the samples do not vary, the four fitting parameters only have small variations with respect to indenter radius and surface orientation, respectively. The defect spacing is found to be one order-of-magnitude larger than the dislocation spacing, as given in Table 1.

When using the above framework for pillar/fiber tests, we note that the stressed volume does not change its size. Therefore, a mere use of the Poisson's distribution in Eq. (14) is in fact equivalent to the weakest link model, and statistical tests are simply sampling the pre-existing defects when the control volume is fixed. Molecular simulations have suggested the strength variation of these heterogeneous dislocation nucleation sources. To this end, a more advanced model by Sudharshan Phani et al. [149] introduced the randomness both in the defect distribution and also in their strengths. One example of the latter is the random distribution of Schmid factor on these sources, as schematically illustrated in Fig. 10(b). Their model will be employed later in Section 4.4.

The unified model in Eq. (17) only addresses the first pop-in event, while a rich spectrum information exists for the subsequent stochastic deformation behavior. To analyze the successive pop-ins under nanoindentation, the essential features in Eq. (17) can be utilized where the evolution of the pre-existing defects or heterogeneous dislocation nucleation sources have to be determined [72]. Contact analysis and molecular simulations have determined the dependence of nucleated dislocation loops on indentation direction [89,130,151], but the evolution of source density is still not fully explored. Ngan et al. [20,21,152] have investigated the relationship between the burst emission rate and the applied strain, and predicted the dependence of stochastic deformation on the number of sources and the stress dependence of the efficiency of the sources. Their recent experimental work [153] made a close comparison among model predictions, experimental strength measurements, and characterization of preexisting dislocation nucleation sources in pre-strained aluminum samples. 


\section{Case Studies from our Size-Dependence Master Plot}

With the master plot in Fig. 8 and the unified model in Section 3, we now present a number of case studies in which experimental studies can intentionally tune the stressed volume size, or the pre-existing defect density, or the surface processing methods. Focus will be placed on how to appropriately interpret these experimental results.

\subsection{Investigations of FIB-milling effects}

The most significant contrast between our experiments and the literature work in Fig. 1(a) is that the spatial stochasticity is not completely probed in the prior micro-pillar tests. These literature results have strength measurements clustered into narrow bands that have a slope in the range of -1.0 -0.5. We note two main factors that contribute to this discrepancy, one being the small stressed volume in these pillar tests since wire tension tests show dramatic scatter, and the other being the potential damages introduced by FIB milling. In this regard, Bei et al. [154] and Shim et al. [155] have designed experiments that intentionally introduce FIB damages, as summarized in Figs. 11 and 12. For the nanoindentation pop-in, two types of FIB milling are presented in Fig. 11(a) with two different incidence angles. For Mo <001> single crystals, the corresponding load-displacement curves are compared in Fig. 11(b) for as grown sample and the two FIB-milled samples. Pop-ins occur at lower load and sometimes cannot be visibly identified for the FIB-milled samples. It is anticipated that the energy particles will introduce vacancy or interstitial loops near the surface. Thus upon FIB milling, the nanoindentation pop-in responses will fall into the range of (C)-(D) in Fig. 8. Furthermore, Fig. 11(c) shows the effect of different degrees of applied voltage: the more damages introduced by FIB, the higher the nanoindentation hardness as this is the commonly known irradiation hardening.

The work in Bei et al. [154] only presented the FIB milling with normal incidence, and suggested that prior works on micro-pillar tests were affected dramatically by the FIB process. This conclusion immediately agitated the micro-pillar test community and drew sharp criticisms from a comment by [156] to the paper by Bei et al. [154]. The following work by Shim et al. [155], in our view, has unambiguously supported the statement in [154]. As shown in Fig. 12, one directionally solidified Mo alloy pillar was intentionally milled by FIB, following exactly the same procedure as in literature work. Note that the as-grown Mo alloy pillars do not show any size effect, as given in Fig. 1(c). Upon FIB milling, the stress-strain curves are given in Figs. 
12(b) and 12(c). Not only the strength now shows some scatter, but also these measurements qualitatively agree with the pre-strained Mo alloy fibers, which is another way to intentionally introduce defects. Further comparison to the FIB-milled Mo pillars [22] in Fig. 12(c) again shows the similar deformation behavior. Should we put these measurements on the master plot in Fig. 8, they would have fallen into the range of (C)-(D). In summary, the vast evidence provided in this review paper suggests that the literature work in Fig. 1(a) that mostly used FIB-milling actually tests a damaged pillar, as depicted in Fig. 12(d), and these tests will not be able to cover the entire range in the master plot in Fig. 8.

\subsection{Investigations of surface polishing effects}

One complicacy in nanoindentation pop-in tests is the surface polishing condition, which also affects prior studies on indentation size effects [10]. Wang et al. [157] have systematically studied several polishing methods: electropolishing, mechanical polishing with $\mathrm{Al}_{2} \mathrm{O}_{3}$ particles, and mechanical polishing followed by colloidal $\mathrm{SiO}_{2}$ polishing. The removal rate in the last method is very slow, so a number of polishing times are presented in Fig. 13. Electropolished samples show clear pop-ins at high pop-in stress, and the corresponding cumulative pop-in curve is very steep, corresponding to the state (A) in Fig. 8. Mechanically polished samples usually do not show clear pop-ins. When using the slow colloidal silica polishing, the longer the polishing time, the closer the cumulative probability curve moves to the electropolished case, corresponding to the transition from state $(\mathrm{C})$ to state $(\mathrm{A})$.

From our unified model in Eq. (17), we can estimate the corresponding defect densities in Fig. 13(b). Curve 1 for electropolished samples has a defect density of $\rho_{\text {defect }}<0.01 \mu \mathrm{m}^{-3}$, Curve 7 has $\rho_{\text {defect }} \approx 1 \mu \mathrm{m}^{-3}$, and Curves 3-5 have $\rho_{\text {defect }}$ in the range of $10-80 \mu \mathrm{m}^{-3}$ which is similar to the pre-strained samples in Table 1.

\subsection{Deconvolution of strain hardening from strength statistics}

The master plot in Fig. 8 suggests a variety of ways to tune and shift these cumulativeprobability contour curves. For example, pre-straining will lead to strain hardening for the bulk strength, so to move up the bulk asymptote. However, pre-straining also increases of the defect density, $\rho_{\text {defect }}$. It is therefore anticipated that, if Fig. 8 is plotted against the stressed volume size, the $10 \% / 90 \%$ curves will not only shift up at the right asymptote, but also move left in the abscissa direction. This line of argument has been proved in a recent work [37]. 
As shown in Fig. 14(a), for the annealed Mo <001> single crystals, the nanoindentation pop-in data with respect to the indenter radius cover the range of (A)-(C) in Fig. 8, as also evidenced from the corresponding cumulative pop-in probability curves in Fig. 14(b). The prestrained samples lead to lower pop-in stress in Fig. 14(a), seemingly suggesting a "strain softening" behavior. Moreover, the 5\% pre-strained results seemingly suggest a linear relationship with a slope of about -0.6 in this logarithmic plot. In fact, because of the increase of defect density, the pop-in data for pre-strain samples now correspond to the range of (B)-(C) in Fig. 8, as also supported by the plots in Figs. 14(c) and 14(d). Using the unified model in Eq. (17) and also considering that $\tau_{\text {defect }}$ depends on the pre-strain level, we can plot the pop-in stress data with respect to a normalized parameter, $R \rho_{\text {defect }}^{1 / 3}$ in Fig. 15 . The inset indeed shows that the bulk strength varies in the range of 600 800 MPa with respect to the pre-strain level. This variation of strain hardening at bulk limit is significantly smaller than the vast contrast between bulk strength (600 800 MPa) and theoretical strength (16.1 GPa here). This example shows that when multiple mechanisms contribute to the small scale mechanical testing, i.e., thermal activation, spatial stochasticity, and strain hardening, the only way to uniquely separate them is to use the unified model in Eq. (17) to analyze the strength statistics. It also suggests that a small set of strength data can never be trusted in making conclusions on these deformations, e.g., "strain softening" in Fig. 14(a) is obviously incorrect and the slope of about -0.6 for the 5\% prestrained data in Fig. 14(a) is also meaningless.

\subsection{Deconvolution of strain rate sensitivity from strength statistics}

The other example of deconvoluting multiple deformation mechanisms is presented in Fig. 16, where directionally solidified Mo alloy fibers are tested in tension with a number of applied strain rates. The testing apparatus is a MEMS-based structure that allows in situ SEM studies. As explained before in Fig. 7, for long fibers, it is almost impossible to reach the upper asymptote because of the large stressed volume. Therefore, the convoluted mechanisms here include the spatial stochasticity and strain rate sensitivity. The collected data in Fig. 16(b) shows considerable scatter, while a general trend of strain rate dependence can be seen. Since the stressed volume has a fixed size but the defect density ( $\rho_{1 D}$ in 1D fashion) may vary, we adopt the model by Sudharshan Phani et al. [149] that provides the 95\% probability as upper envelope and the $5 \%$ probability as the lower envelope. As explained in Section 3.2, in addition to the 
defect density, a variation in the Schmid factor in $\left[S_{\min }, S_{\max }\right]$ is also introduced. As discussed before in Fig. 5, we note that fiber test will never reach the theoretical strength $\sigma_{t h}=\tau_{t h} / S$, so we use $\sigma_{\max }^{\text {pillar }}$ to denote the upper asymptote. As explained before, $\sigma_{\max }^{\text {pillar }} \approx \sigma_{t h} / 3$. Thus,

$$
\begin{gathered}
\sigma_{95 \%}=\sigma_{\max }^{\text {pillar }}, \text { for } \rho_{1 D} l \leq \frac{\ln \beta}{\frac{S_{\min }}{S_{\max }}-1}, \\
\sigma_{95 \%}=\sigma_{\text {bulk }}\left(\frac{\rho_{1 D} L}{\rho_{1 D} L+\ln \beta}\right) \text {, for } \rho_{1 D} l>\frac{\ln \beta}{\frac{S_{\min }}{S_{\max }}-1}, \\
\sigma_{5 \%}=\sigma_{\max }^{\text {pillar }}, \text { for } \rho_{1 D} l \leq \frac{\ln (1-\beta)}{\frac{S_{\min }}{S_{\max }}-1}, \\
\sigma_{5 \%}=\sigma_{\text {bulk }}\left(\frac{\rho_{1 D} L}{\rho_{1 D} L+\ln (1-\beta)}\right), \text { for } \rho_{1 D} l>\frac{\ln (1-\beta)}{\frac{S_{\min }}{S_{\max }}-1},
\end{gathered}
$$

where $\beta$ is the strength probability of lower bound (e.g., 5\%). Note that this model does not

include the thermal activation near $\sigma_{\max }^{\text {pillar }}$, because the measured strengths are much lower than this upper asymptote.

\subsection{What strength values should we trust for pillar/fiber/whisker tests?}

We now have seen a variety of compression and tension tests on short pillars and long fibers. Results in Figs. 1(c) and 6 are presented with respect to the sample diameter or edge width. Here following the presentation in Sudharshan Phani et al. [44,149,150], Fig. 17(a) summarized the tests on as-grown Mo alloy pillars/fibers with respect to the sample length. Note all these results in Fig. 17(a) have a sample edge with of about $360 \mathrm{~nm}$, except the thinner samples of $160 \mathrm{~nm}$ in Chisholm et al. [45]. Using the model in Eq. (18), the normal yield strength is plotted against the normalized sample length, $\rho_{1 D} L$, which resembles the master plot for nanoindentation pop-in in Fig. 15. The lower asymptote is the bulk strength of as-grown Mo alloy fiber, while the $16 \%$ pre-strain samples have a higher bulk strength, i.e., strain hardening as previously demonstrated in the inset in Fig. 15. Consequently, a conclusive set of small scale mechanical measurements on fibers can only be obtained with (i) a variation of sample lengths by one or two orders of magnitude and (ii) a sufficient number of tests for statistical variations. On the other hand, the variation of defect density is optional, since highly defected fibers cannot really reach the upper asymptote. 
Brenner $[158,159]$ measured the strength of $\mathrm{Fe}\langle 111\rangle, \mathrm{Cu}\langle 111\rangle$, and $\mathrm{Ag}\langle 100\rangle$ singlecrystalline whiskers, and found a general trend of "the thicker the weaker". His data are compiled in Fig. 18, together with micro-pillar compression tests on directionally solidified Mo alloy [39,40] and $\mathrm{Zr}$-based BMG [65], and tensile tests on $\mathrm{Zr}_{49} \mathrm{Cu}_{51}$ metallic glass wires (FIBmilled, Tian et al. [160]) and $\mathrm{Cu}<110>$ whiskers prepared by physical vapor deposition (Richter et al. [161]). From Ogata et al. [38], we find the theoretical strength for Fe $\{110\}\langle\overline{1} 11\rangle$ slip system is $8.14 \mathrm{GPa}$ (or $0.106 G$ ), that for $\mathrm{Cu}\{111\}\langle 11 \overline{2}\rangle$ is $2.16 \mathrm{GPa}$ (or $0.07 G$ ), and that for $\mathrm{Ag}$ $\{111\}\langle 11 \overline{2}\rangle$ is $1.65 \mathrm{GPa}(0.066 G)$. These theoretical values, together with the Schmid factor calculation since the whisker direction is given, will give the predicted theoretical strength in normal direction, $\sigma_{t h}$. Data plotted in Fig. 18 for $\sigma_{t h}$ are much higher than the measured whisker strengths. Brenner [158] argue that the theoretical shear strength should fall into a range of $G / 30 \sim G / 100$, since the prediction from idealized Frenkel sinusoid, $G / 2 \pi$, is a significant overestimate. However, first principles calculations Ogata et al. [38] give a range of $G / 10 \sim G / 15$ for these metals. To resolve this discrepancy, we note that the uniaxial tests will never reach the theoretical strength; rather, $\sigma_{\max }^{\text {fiber }} \approx \frac{1}{3} \sigma_{t h}$ from Fig. 5. Therefore, using $\sigma_{\max }^{\text {fiber }}$ as the upper asymptote, Brenner's data are found to perfectly follow the cumulative-probability contour plots, similar to the master plot in Figs. 8 and 17. Areas enveloped by the 10\%/90\% cumulative probability contours are indicated by the shaded domains. The bulk strengths of these materials are very low, so the lower asymptotes are not shown. These samples are roughly $4 \mathrm{~mm}$ in length. Brenner [158] also observed that if the fractured samples were tested again, the strength increased. In his era when metallic wires are very defective, da Vinci noted "the longer the weaker", exactly as Brenner's observations. Ancient Chinese philosophers in Confucius era commonly used a metaphor that a dam of thousands miles long may collapse because of one ant hole. All these are perfect demonstrations of the weakest link model.

Data from Bharathula and Flores [65] are included in Fig. 18 with a lower bound strength of $1.85 \mathrm{GPa}$. The overall trends of these data suggest that the measurements are still in the range of (C)-(D) in Fig. 8. Now what is the upper limit of the tensile strength of metallic glasses? From nanoindentation pop-in tests in Fig. 4, we obtain a theoretical shear strength of about $3.8 \mathrm{GPa}$. Converting this value to normal strength using Mises plasticity (a factor of $\sqrt{3}$ difference) and 
also considering the reduction of $\sigma_{\max }^{\text {fiber }}$ from $\sigma_{t h}$, we again find that these measurements by Bharathula and Flores [65] begin to transition to the upper asymptote of $\sigma_{\max }^{\text {fiber }}$ of about $3.3 \mathrm{GPa}$. Tian et al. [160] conducted tensile tests of FIB-milled $\mathrm{Zr}_{49} \mathrm{Cu}_{51}$ metallic glasses fibers, and found a range of 3 3.8 GPa for fiber diameters in the range of $210 \sim 300 \mathrm{~nm}$, as given in the purple rectangle in Fig. 18. They concluded that the ideal elastic limit and the normal theoretical strength are reached. However, we believe that they actually did not. From our nanoindentation pop-in test, the ideal strength should be much higher than their measured values. In other words, their strength values are still 1/3 1/2 of the ideal normal strength because of the surface effects, similar to our data processing for the measurements by Bharathula and Flores [65] in Fig. 18.

Reaching the asymptote of $\sigma_{\max }^{\text {fiber }}$, as shown by experiments in Figs. 15 and 17, can be achieved by reducing the stressed volume size and also by removing pre-existing defects, such as annealing and then tensile tests in Lowry et al. [162]. As suggested in Figs. 11 and 12, avoiding FIB-milling is another promising method. For one example, Richter et al. [161] fabricated $\mathrm{Cu}$ $<110>$ whiskers using the physical vapor deposition technique. These whiskers are very thin (less than about $300 \mathrm{~nm}$ ) and long (about $30 \mu \mathrm{m}$ ). Their fracture strength measurements are found to be in the range of $\sigma_{t h}$ and $\sigma_{\max }^{\text {fiber }} \approx \frac{1}{3} \sigma_{t h}$, which differs from the general trends of other data in Fig. 18. We note that these whiskers have a hexagonal cross-sectional shape, so that a factor of 1/3 might be an underestimate. Also it is conceivable that the fracture strength is higher than the yield strength. For the other example, Jennings et al. [26] fabricated $\mathrm{Cu}<111>$ single crystalline pillars by electroplating, and found an upper bound strength of about $800 \mathrm{MPa}$ (for strain rate of $\left.10^{-3} \mathrm{~s}^{-1}\right), 1200 \mathrm{MPa}\left(10^{-3} \mathrm{~s}^{-1}\right)$, and $1500 \mathrm{MPa}\left(10^{-1} \mathrm{~s}^{-1}\right)$ when pillar diameters are lower than about $120 \mathrm{~nm}$. All these values are much smaller than Brenner's results of about 2.3 $\mathrm{GPa}$ in Fig. 18. Also their measurements in the diameter range of 120 600 nm show a narrow band with a slope of -0.54 . Therefore, findings by Jennings et al. [26] do not appear to agree with the vast of experiments compiled in Fig. 18 and our master plot in Fig. 8.

\subsection{Nanoindentation pop-in as a structural probe in BMG}

Nanoindentation pop-in tests in Fig. 4 provide a way to establish a structure-property relationship in metallic glasses. Fig. 19(a) replots the same data in Fig. 1(b) [51]. Again, we assume that the metallic glass material consists of a pure-glass matrix dispersed with some "weak zones" or "soft spots" where the atomic packing deviates from an ideal glass structure. 
The unified model in Eq. (17) should be modified since there are no slip systems in metallic glasses, and the activation volume model in Eq. (12) is adopted for $\Delta \Pi[93,144]$. Thus we can obtain the defect density $\rho_{\text {defect }}$ in Table I and also in Fig. 19(c). Upon annealing from the as-cast condition, longer annealing time and higher annealing temperature will significantly reduce the defect density. Additionally, $\mathrm{Li}$ et al. [53,57] conducted differential scanning calorimetry, elastic constants, and synchrotron x-ray tests, which show little or negligible dependence on the annealing conditions. These as-cast and annealed samples have different fracture behavior, as shown in the three-point notch bending results in Fig. 19(b). Therefore, a relationship between the fracture energy density obtained from three-point notch bending tests and the defect density can be established in Fig. 19(d), which shows the exponential increase of the fracture energy.

The structure-property relationship established in Fig. 19(d) can be qualitatively explained in Fig. 20. For nanoindentation pop-in tests, the fully annealed samples have little or no soft zones, so that their deformation behavior resemble ideal glass, while as-cast samples exhibit metal-like behavior and sometimes show a certain degree of ductile deformation in the stress-strain curves in tension. The line of argument can be employed in the process zone near a crack tip. With the presence of these defects, the stress singularity at the crack tip can be shielded and thus the crack growth is resisted. For the fully annealed samples, we will obtain the brittle fracture. These results suggest the use of pop-in statistical measurements as a mechanicallybased structural probe. For example, Magagnosc et al. [163] studied the irradiation effects on metallic glasses, wherein the pop-in tests can be employed to provide an indirect study of structural heterogeneity. Yang et al. [164] have used spherical indentation to investigate the anelastic deformation in metallic glasses, as another way to probe the structural heterogeneity, which can certainly be combined with the pop-in statistical tests.

As a hindsight, we note the cumulative probability curves are very close to one another because $\tau_{\text {th }}$ and $\tau_{\text {defect }}$ are far apart. As a consequence, the homogeneous nucleation model in Eq. (10) is often used, rather inappropriately in our view, to explain these pop-in curves. For example, Choi et al. $[165,166]$ measured the cumulative pop-in probabilities curves as these in Fig. 4, and their use of activation volume model concluded a dependence of the activation volume on the annealing condition. Recall the discussion in Fig. 9, and we note that this conclusion is dubious. Our unified model shows that the activation volume, as an intrinsic 
parameter, does not depend on the annealing condition [53,57]. What changes with respect to the annealing condition is the extrinsic defect density.

Lastly, it should be pointed out that we do not an explicit model of the weak zones or soft spots in Fig. 20. In other words, they are treated phenomenologically. Recent works by Fan et al. [167,168] provide key insights along this line of study. Their atomistic simulations have identified and documented the thermally activated processes in a model metallic glass with or without annealing. The number density distribution in Fig. 21(b) shows that the as-quenched sample (less stable system, sys-1) have a high probability of finding thermally activated processes with low energy barrier, while the annealed one (more stable system, sys-2) have a shifted distribution to high energy barrier. The understanding along this line will help develop more rigorous analyses for the strength measurements in metallic glasses. Note that our model in analyzing data in Fig. 4 only use $\tau_{\text {th }}$ and $\tau_{\text {defect }}$, which correspond to two Dirac delta functions on the plot in Fig. 21(b).

\section{Conclusions}

The central topic of this review paper is the development of a new perspective and a complete view on the strength statistics in small stressed volumes. Our master plot in Fig. 8 extends the literature views on searching the scaling exponent in the strength versus size plot (e.g., Fig. 1(a)). A large set of experiments have been complied, and a unified model that convolutes both thermal activation and spatial stochasticity has been developed. Key conclusions are given below.

(1) The strength measurements should approach an upper asymptote at small stressed volume or low defect density (where thermal activation governs the strength statistics), a lower asymptote at the bulk scale, and a transition between these two asymptotes that display significant strength scattering due to the spatial stochasticity.

(2) Nanoindentation pop-in tests (e.g., Figs. 1(b), 3, 4, and 13-15) support the master plot in Fig. 8. Our unified model provides a unique way to deconvolute contributions from multiple deformation mechanisms, such as the strain hardening in Fig. 15 and the strain rate sensitivity in Fig. 16.

(3) Micro-pillar compression tests (e.g., Figs. 1(c), 5, 6, and 12) support the master plot in Fig. 8. As-grown Mo alloy fibers do not show size effects because of the defect free state. 
Different degrees of pre-straining in Fig. 6 or intentional FIB milling in Fig. 12 confirm that the pre-existing defects significantly affect the strength measurements, which thus challenge the commonly accepted view in Fig. 1(a).

(4) Fiber/wire/whisker tension tests (e.g., Figs. 1(c), 7, and 16-18) support the master plot in Fig. 8, and these data usually scatter significantly because of the large stressed volume. Brenner's whisker data are also summarized in Fig. 18. The contrast between $\sigma_{t h}$ and $\sigma_{\max }^{f i b e r}$, as explained in Fig. 5, turns out to be the key factor that rationalize these experiments. It is found that $\sigma_{\max }^{\text {fiber }} / \sigma_{t h}$ should fall into a range of about [1/3,1/2].

(5) Small scale mechanical tests should cover a wide range of scale spectrum; otherwise, incorrect conclusions will be derived. This can be achieved by changing the stressed volume size via the change of indenter radius, pillar diameter, and fiber length, or by changing the pre-existing defect by pre-straining or FIB milling, or by changing the crystallographic orientation and thus the indentation Schmid factor.

(6) The major limitation of this study is the lack of an explicit model of the heterogeneous dislocation nucleation sources or the structural heterogeneity. Rather, only a weakestlink-type model is adopted to determine the probability of finding such defects. Also we focus on the first pop-in or the initial yield, while the subsequent deformation behavior involves complicated evolution of defect microstructures.

\section{Acknowledgements}

This work was sponsored by the U.S. Department of Energy, Office of Science, Basic Energy Sciences, Materials Sciences and Engineering Division. 


\section{References}

[1] Basic Energy Sciences Advisory Committee (BESAC). Basic Research Needs to Assure a Secure Energy Future, a report for the Office of Science, U.S. Department of Energy, 2003.

[2] Song G, Sun Z, Li L, Xu X, Rawlings M, Liebscher CH, Clausen B, Poplawsky J, Leonard DN, Huang S, Teng Z, Liu CT, Asta MD, Gao YF, Dunand DC, Ghosh G, Chen MW, Fine ME, Liaw PK. Ferritic alloy with extreme creep resistance via coherent hierarchical precipitates. Scientific Reports 2015:5:16327.

[3] Sawyer WG, Argibay N, Burris DL, Krick BA. Mechanistic studies in friction and wear of bulk materials. Annu. Rev. Mater. Res. 2014:44:395-427.

[4] Wang RZ, Gupta H. Deformation and fracture mechanisms of bone and nacre. Annu. Rev. Mater. Res. 2011:41:41-73.

[5] Zinkle SJ, Snead LL. Designing radiation resistance in materials for fusion energy. Annu. Rev. Mater. Res. 2014:44:241-267.

[6] Freund LB, Suresh S. Thin Film Materials: stress, defect formation and surface evolution. Cambridge University Press, UK, 2003.

[7] Evans AG, Mumm DR, Hutchinson JW, Meier GH, Pettit FS. Mechanisms controlling the durability of thermal barrier coatings. Prog. Mater. Sci. 2001:46:505-553.

[8] Nix WD, Gao H. Indentation size effects in crystalline materials: a law for strain gradient plasticity. J. Mech. Phys. Solids 1998:46:411-425.

[9] Nix WD, Greer JR, Feng G, Lilleodden ET. Deformation at the nanometer and micrometer length scales: effects of strain gradients and dislocation starvation. Thin Solid Films 2007:515:3152-3157.

[10] Pharr GM, Herbert EG, Gao YF. The indentation size effect: a critical examination of experimental observations and mechanistic interpretations. Ann. Rev. Mater. Res. 2010:40:271-290.

[11] Zhang YH, Gao YF, Nicola L. Lattice rotation caused by wedge indentation of a single crystal: dislocation dynamics compared to crystal plasticity simulations. J. Mech. Phys. Solids 2014:68:267-279.

[12] Gao YF, Larson BC, Lee JH, Nicola L, Tischler JZ, Pharr GM. Lattice rotation patterns and strain gradient effects in face-centered-cubic single crystals under spherical indentation. J. Appl. Mech. 2015:82:061007.

[13] Uchic MD, Dimiduk DM, Florando JN, Nix WD. Sample dimensions influence strength and crystal plasticity. Science 2004:305:986-989.

[14] Dimiduk DM, Uchic MD, Parthasarathy TA. Size-affected single-slip behavior of pure nickel microcrystals. Acta Mater. 2005:53:4065-4077.

[15] Uchic MD, Shade PA, Dimiduk DM. Plasticity of micrometer-scale single crystals in compression. Annu. Rev. Mater. Res. 2009:39:361-386.

[16] Zhu T, Li J. Ultra-strength materials. Prog. Mater. Sci. 2010:55:710-757.

[17] Greer JR, de Hosson JTM. Plasticity in small-sized metallic systems: intrinsic versus extrinsic size effect. Prog. Mater. Sci. 2011:56:654-724.

[18] Greer JR, Oliver WC, Nix WD. Size dependence of mechanical properties of gold at the micron scale in the absence of strain gradients. Acta Mater. 2015:53:1821-1830.

[19] Volkert CA, Lilleodden ET. Size effects in the deformation of sub-micron Au columns. Phil. Mag. 2006:86:5567-5579. 
[20] Ng KS, Ngan AHW. Stochastic nature of plasticity of aluminum micro-pillars. Acta Mater. 2008:56:1712-1720.

[21] Ng KS, Ngan AHW. Stochastic theory for jerky deformation in small crystal volumes with pre-existing dislocations. Phil. Mag. 2008:88:677-688.

[22] Brinckmann S, Kim JY, Greer JR. Fundamental differences in mechanical behavior between two types of crystals at nano-scale. Phys. Rev. Lett. 2008:100:155502.

[23] Jennings AT, Burek MJ, Greer JR. Microstructure versus size: mechanical properties of electroplated single crystalline Cu nanopillars. Phys. Rev. Lett. 2010:104:135503.

[24] Ngan AHW. An explanation for the power-law scaling of size effect on strength in micro-specimens. Scripta Mater. 2011:65:978-981.

[25] Kiener D, Minor AM. Source truncation and exhaustion: insights from quantitative in situ TEM tensile testing. Nano Lett. 2011:11:3816-3820.

[26] Jennings AT, Li J, Greer JR. Emergence of strain-rate sensitivity in Cu nanopillars: transition from dislocation multiplication to dislocation nucleation. Acta Mater. 2011:59:5627-5637.

[27] Han SM, Bozorg-Grayeli T, Groves JR, Nix WD. Size effects on strength and plasticity of vanadium nanopillars. Scripta Mater. 2010:63:1153-1156.

[28] Kim JY, Jang D, Greer JR. Crystallographic orientation and size dependence of tensioncompression asymmetry in molybdenum nnao-pillars. Int. J. Plast. 2012:28:46-52.

[29] Espinosa HD, Panico M, Berbenni S, Schwarz KW. Discrete dislocation dynamics simulations to interpret plasticity size and surface effects in freestanding FCC thin films. Int. J. Plast. 2006:22:2091-2117.

[30] Tang H, Schwarz KW, Espinosa HD. Dislocation-source shutdown and the plastic behavior of single-crystal micropillars. Phys. Rev. Lett. 2008:100:185503.

[31] Weinberger CR, Cai W. The stability of Lomer-Cottrell jogs in nanopillars. Scripta Mater. 2011:64:529-532.

[32] Zhu Y, Xiang Y. A continuum model for dislocation dynamics in three dimensions using the dislocation density potential functions and its application to micro-pillars. J. Mech. Phys. Solids 2015:84:230-253.

[33] Ryu I, Cai W, Nix WD, Gao H. Stochastic behaviors in plastic deformation of facecentered cubic micropillars governed by surface nucleation and truncated source operation. Acta Mater. 2015:95:176-183.

[34] Guruprasad PJ, Benzerga AA. Size effects under homogenous deformation of single crytals: a discrete dislocation analysis. J. Mech. Phys. Solids 2008:56:132-156.

[35] Benzerga AA. Micro-pillar plasticity: 2.5D mesoscopic simulations. J. Mech. Phys. Solids 2009:57:1459-1469.

[36] Li TL, Bei H, Morris JR, George EP, Gao YF. Scale effects in convoluted thermal/spatial statistics of plasticity initiation in small stressed volumes during nanoindentation. Mater. Sci. Tech. 2012:28:1055-1059.

[37] Bei H, Xia YZ, Barabash RI, Gao YF. A tale of two mechanisms: strain-softening versus strain-hardening in single crystals under small stressed volumes. Scripta Mater. 2016:110:48-52.

[38] Ogata S, Li J, Hirosaki N, Shibutani Y, Yip S. Ideal shear strain of metals and ceramics. Phys. Rev. B 2004:70:104104. 
[39] Bei H, Shim S, George EP, Miller MK, Herbert EG, Pharr GM. Compressive strengths of molybdenum alloy micro-pillars prepared using a new technique. Scripta Mater. 2007:57:397.

[40] Bei H, Shim S, Pharr GM, George EP. Effects of pre-strain on the compressive stressstrain response of Mo-alloy single crystal micro-pillars. Acta Mater. 2008:56:4762.

[41] Bei H, George EP, Kenik EA, Pharr GM. Directional solidification and microstructures of near-eutectic Cr-Cr 3 Si alloys. Acta Mater. 2003:51:6241-6252.

[42] Bei H, George EP. Microstructures and mechanical properties of a directionally solidified NiAl-Mo eutectic alloy. Acta Mater. 2005:53:69-77.

[43] Bei H, George EP. Microstructures and mechanical properties of directionally solidified intermetallic composites. Mater. Sci. Forum 2007:539-543:1495-1500.

[44] Sudharshan Phani P, Johanns KE, Duscher G, Gali A, George EP, Pharr GM. Scanning transmission electron microscope observations of defects in as-grown and pre-strained Mo alloy fibers. Acta Mater. 2011:59:2172-2179.

[45] Chisholm C, Bei H, Lowry MB, Oh J, Syed Asif SA, Warren OL, Shan ZW, George EP, Minor AM. Dislocation starvation and exhaustion hardening in Mo alloy nanofibers. Acta Mater. 2012:60:2258-2264.

[46] Loya PE, Xia YZ, Peng C, Bei H, Zhang P, Zhang J, George EP, Gao YF, Lou J. Yield strength dependence on strain rate of molybdenum-alloy nanofibers. Appl. Phys. Lett. 2014:104:251909.

[47] Schuh CA, Hufnagel TC, Ramamurty U. Mechanical behavior of amorphous alloys. Acta Mater. 2007:55:4067-4109.

[48] Yang Y, Ye J, Lu J, Gao YF, Liaw PK. Metallic glasses: gaining plasticity for microsystems. JOM 2010:62(2):93-98.

[49] Jia HL, Liu FX, An ZN, Li WD, Wang GY, Chu JP, Jang JSC, Gao YF, Liaw PK. Thinfilm metallic glasses for substrate fatigue-property improvements. Thin Solid Films 2014:561:2-27.

[50] Schuh CA, Nieh TG. A nanoindentation study of serrated flow in bulk metallic glasses. Acta Mater. 2003:51:87-99

[51] Bei H, Lu ZP, Shim S, Chen G, George EP. Specimen size effects on Zr-based bulk metallic glasses investigated by uniaxial compression and spherical nanoindentation. Metall. Mater. Trans. A 2010:41A:1735-1742.

[52] Gao YF, Wang L, Bei H, Nieh TG. On the shear band direction in metallic glasses. Acta Mater. 2011:59:4159-4167.

[53] Li WD, Gao YF, Bei H. On the correlation between microscopic structural heterogeneity and embrittlement behavior in metallic glasses. Scientific Reports 2015:5:14786.

[54] Gao YF, Yang B, Nieh TG. Thermomechanical instability analysis of inhomogeneous deformation in amorphous alloys. Acta Mater. 2007:55:2319-2327.

[55] Cheng YQ, Ma E. Intrinsic shear strength of metallic glass. Acta Mater. 2011:59:18001807.

[56] Cheng YQ, Ma E. Atomic-level structure and structure-property relationship in metallic glass. Prog. Mater. Sci. 2011:56:379-473.

[57] Li WD, Bei H, Tong Y, Dmowski W, Gao YF. Structural heterogeneity induced plasticity in bulk metallic glasses: From well-relaxed fragile glass to metal-like behavior. Appl.

Phys. Lett. 2013:103:171910. 
[58] Volkert CA, Donohue A, Spaepen F. Effect of sample size on deformation in amorphous metals. J. Appl. Phys. 2008:103:083539.

[59] Xie S, George EP. Size-dependent plasticity and fracture of a metallic glass in compression. Intermetallics 2008:16:485-489.

[60] Jang D, Gross CT, Greer JR. Effects of size on the strength and deformation mechanism in Zr-based metallic glasses. Int. J. Plast. 2011:27:858-867.

[61] Wang CC, Ding J, Cheng YQ, Wan JC, Tian L, Sun J, Shan ZW, Li J, Ma E. Sample size matters for $\mathrm{Al}_{88} \mathrm{Fe}_{7} \mathrm{Gd}_{5}$ metallic glass: smaller is stronger. Acta Mater. 2012:60:53705379.

[62] Kuzmin OV, Pei YT, Chen CQ, de Hosson JTM. Intrinsic and extrinsic size effects in the deformation of metallic glass nanopillars. Acta Mater. 2012:60:889-898.

[63] Kuzmin OV, Pei YT, de Hosson JTM. Size effects and ductility of Al-based metallic glass. Scripta Mater. 2012:67:344-347.

[64] Liu MC, Huang JC, Chen KW, Lin JF, Li WD, Gao YF, Nieh TG. Is the compression of tapered micro and nanopillar samples a legitimate technique for the identification of deformation mode change in metallic glasses? Scripta Mater. 2012:66:817-820.

[65] Bharathula A, Flores KM. Variability in the yield strength of a metallic glass at micron and submicron length scales. Acta Mater. 2011:59:7199-7205.

[66] Oliver WC, Pharr GM. An improved technique for determining hardness and elastic modulus using load and displacement sensing indentation experiments. J. Mater. Res. 1992:7:1564-1583.

[67] Oliver WC, Pharr GM. Measurement of hardness and elastic modulus by instrumented indentation: Advances in understanding and refinements to methodology. J. Mater. Res. 2004:19:3-20.

[68] Lee JH, Gao YF, Johanns KE, Pharr GM. Cohesive interface simulations of indentation cracking as a fracture toughness measurement method for brittle materials. Acta Mater. 2012:60:5448-5467.

[69] Lodes MA, Hartmaier A, Göken M, Durst K. Influence of dislocation density on the popin behavior and indentation size effect in $\mathrm{CaF}_{2}$ single crystals: experiments and molecular dynamics simulations. Acta Mater. 2011:59:4264-4273.

[70] Durst K, Backes B, Franke O, Göken M. Indentation size effect in metallic materials: modeling strength from pop-in to macroscopic hardness using geometrically necessary dislocations. Acta Mater. 2006:54:2547-2555.

[71] Durst K, Göken M, Pharr GM. Indentation size effect in spherical and pyramidal indentations. J. Phys. D. Appl. Phys. 2008:41:074005.

[72] Xia YZ, Gao YF, Pharr GM, Bei H. Single versus successive pop-in modes in nanoindentation tests of single crystals. J. Mater. Res. In press.

[73] Johnson KL. Contact Mechanics. Cambridge University Press, UK, 1985.

[74] Willis JR. Hertzian contact of anisotropic bodies. J. Mech. Phys. Solids 1966:14:163-176.

[75] Vlassak JJ, Nix WD. Indentation modulus of elastically anisotropic half spaces. Phil. Mag. A 1993:67:1045-1056.

[76] Swadener JG, Pharr GM. Indentation of elastically anisotropic half-spaces by cones and parabola of revolution. Phil. Mag. A 2001:81:447-466.

[77] Vlassak JJ, Ciavarella M, Barber JR, Wang X. The indentation modulus of elastically anisotropic materials for indenters of arbitrary shape. J. Mech. Phys. Solids 2003:51:1701-1721. 
[78] Gao YF, Pharr GM. Multidimensional contact moduli of elastically anisotropic solids. Scripta Mater. 2007:57:13-16.

[79] Gao YF, Xu HT, Oliver WC, Pharr GM. Effective elastic modulus of film-on-substrate systems under normal and tangential contacts. J. Mech. Phys. Solids 2008:56:402-416.

[80] Li TL, Lee JH, Gao YF. An approximate formulation of the effective indentation modulus of elastically anisotropic film-on-substrate system. Int. J. Appl. Mech. 2009:1:515-525.

[81] Li TL, Gao YF, Bei H, George EP. Indentation Schmid factor and orientation dependence of nanoindentation pop-in behavior of NiAl single crystals. J. Mech. Phys. Solids 2011:59:1147-1162.

[82] Annett J, Gao YF, Cross GLW, Herbert EG, Lucas BN. Mesoscale friction anisotropy revealed by slidingless tests. J. Mater. Res. 2011:26:2373-2378.

[83] Lee JH, Gao YF, Pharr GM. Effective Poisson's ratio from combined normal and lateral contacts of single crystals. J. Mater. Res. 2012:27:182-191.

[84] Gao YF, Larson BC. Displacement fields and self-energies of circular and polygonal dislocation loops in homogeneous and layered anisotropic solids. J. Mech. Phys. Solids 2015:83:104-128.

[85] Li WD, Bei H, Qu J, Gao YF. Effects of machine stiffness on the loading-displacement curve during spherical nano-indentation. J. Mater. Res. 2013:28:1903-1911.

[86] Corcoran SG, Colton RJ, Lilleodden ET, Gerberich WW. Anomalous plastic deformation at surfaces: nanoindentation of gold single crystals. Phys. Rev. B 1997:55:R16057R16060.

[87] Shim S, Bei H, George EP, Pharr GM. A different type of indentation size effect. Scripta Mater. 2008:59:1095-1098.

[88] Cordill MJ, Moody NR, Gerberich WW. The role of dislocation walls for nanoindentation to shallow depths. Int. J. Plast. 2009:25:281-301.

[89] Wang L, Bei H, Li TL, Gao YF, George EP, Nieh TG. Determining the activation energies and slip systems for dislocation nucleation in body-centered cubic Mo and facecentered cubic Ni single crystals. Scripta Mater. 2011:65:179-182.

[90] Minor AM, Syed Asif SA, Shan Z, Stach EA, Cyrankowski E, Wyrobek TJ, Warren OL. A new view of the onset of plasticity during the nanoindentation of aluminum. Nature Mater. 2006:5:697-702.

[91] Bei H, Gao YF, Shim S, George EP, Pharr GM. Strength differences arising from homogenous versus heterogeneous dislocation nucleation. Phys. Rev. B 2008:77:060103(R).

[92] Wu D, Morris JR, Nieh TG. Effect of tip radius on the incipient plasticity of chromium studied by nanoindentation. Scripta Mat. 2015:94:52-55.

[93] Schuh CA, Lund AC. Application of nucleation theory to the rate dependence of incipient plasticity during nanoindentation. J. Mater. Res. 2004:19:2152-2158.

[94] Schuh CA, Mason JK, Lund AC. Quantitative insight into dislocation nucleation from high-temperature nanoindentation experiments. Nat. Mater. 2005:4:617-621.

[95] Mason JK, Lund AC, Schuh CA. Determining the activation energy and volume for the onset of plasticity during nanoindentation. Phys. Rev. B 2006:73:054102.

[96] Kwon J, Brandes MC, Sudharshan Phani P, Pilchak AP, Gao YF, George EP, Pharr GM, Mills MJ. Characterization of deformation anisotropies in an $\alpha$-Ti alloy by nanoindentation and electron microscopy. Acta Mater. 2013:61:4743-4756. 
[97] Somekawa H, Schuh CA. Effect of solid solution elements on nanoindentation hardness, rate dependence, and incipient plasticity in fine grained magnesium alloys. Acta Mater. 2011:59:7554-7563.

[98] Catoor D, Gao YF, Geng J, Prasad MJNV, Herbert EG, Kumar KS, Pharr GM, George EP. Incipient plasticity and deformation mechanisms in single-crystal $\mathrm{Mg}$ during spherical nanoindentation. Acta Mater. 2013:61:2953-2965.

[99] Guo T, Siska F, Barnett MR. Distinguishing between slip and twinning events during nanoindentation of magnesium alloy AZ31. Scripta Mater. 2016:110:10-13.

[100] Xia YZ, Bei H, Gao YF, Catoor D, George EP. Synthesis, characterization, and nanoindentation response of single crystal $\mathrm{Fe}-\mathrm{Cr}-\mathrm{Ni}$ alloys with $\mathrm{FCC}$ and $\mathrm{BCC}$ structures. Mater. Sci. Eng. A 2014:611:177-187.

[101] Gerberich WW, Nelson JC, Lilleodden ET, Anderson P, Wyrobek JT. Indentation induced dislocation nucleation: the initial yield point. Acta Mater. 1996:44:3585-3598.

[102] Page TF, Oliver WC, McHargue CJ. The deformation-behavior of ceramic crystals subjected to very low load (nano)indentations. J. Mater. Res. 1992:7:450-473.

[103] Bei H, George EP, Hay JL, Pharr GM. Influence of indenter tip geometry on elastic deformation during nanoindentation. Phys. Rev. Lett. 2005:95:045501.

[104] Jang JI, Bei H, Becher PF, Pharr GM. Experimental analysis of the elastic-plastic transition during nanoindentation of single crystal $\alpha$-silicon nitride. J. Am. Ceram. Soc. 2012:95:2113-2115.

[105] Venkataraman SK, Kohlstedt DL, Gerberich WW. Continuous microindentation of passivating surfaces. J. Mater. Res. 1993:8:685-688.

[106] Jungk JM, Boyce BL, Buchheit TE, Friedmann TA, Yang D, Gerberich WW. Indentation fracture toughness and acoustic energy release in tetrahedral amorphous carbon diamondlike thin films. Acta Mater. 2006:54:4043-4052.

[107] Li J, van Vliet KJ, Zhu T, Yip S, Suresh S. Atomistic mechanisms governing elastic limit and incipient plasticity in crystals. Nature 2002:418:307-310.

[108] Lilleodden ET, Zimmerman JA, Foiles SM, Nix WD. Atomistic simulations of elastic deformation and dislocation nucleation during nanoindentation. J. Mech. Phys. Solids 2003:51:901-920.

[109] Zhu T, Li J, van Vliet KJ, Ogata S, Yip S, Suresh S. Predictive modeling of nanoindentation-induced homogeneous dislocation nucleation in copper. J. Mech. Phys. Solids 2004:52:691-724.

[110] Begau C, Hartmaier A, George EP, Pharr GM. Atomistic processes of dislocation generation and plastic deformation during nanoindentation. Acta Mater. 2011:59:934942.

[111] Rabkin E, Srolovitz DJ. Onset of plasticity in gold nanopillar compression. Nano Lett. 2007:7:101-107.

[112] Rabkin E, Nam HS, Srolovitz DJ. Atomistic simulation of the deformation of gold nanopillars. Acta Mater. 2007:55:2085-2099.

[113] Zhu T, Li J, Samanta A, Leach A, Gall K. Temperature and strain-rate dependence of surface dislocation nucleation. Phys. Rev. Lett. 2008:100:025502.

[114] Zuo L, Ngan AHW. Molecular dynamics study on compressive yield strength in $\mathrm{Ni}_{3} \mathrm{Al}$ micro-pillars. Phil. Mag. Lett. 2006:86:355. 
[115] Mordehai D, Kazakevich M, Srolovitz DJ, Rabkin E. Nanoindentation size effect in single-crystal nanoparticles and thin films: a comparative experimental and simulation study. Acta Mater. 2011:59:2309-2321.

[116] Chen LY, He MR, Shin J, Richter G, Gianola DS. Measuring surface dislocation nucleation in defect-scarce nanostructures. Nat. Mater. 2015:14:707.

[117] Wagner AJ, Hintsala ED, Kumar P, Gerberich WW, Mkhoyan KA. Mechanisms of plasticity in near-theoretical strength sub-100 nm Si nanocubes. Acta Mater. 2015:100:256-265.

[118] Barabash RI, Bei H, Gao YF, Ice GE. Indentation-induced localized deformation and elastic strain partitioning in composites at submicron length scale. Acta Mater. 2010:58:6784-6789.

[119] Barabash RI, Bei H, Gao YF, Ice GE, George EP. 3D X-ray microprobe investigation of local dislocation densities and elastic strain gradients in a NiAl-Mo composite and exposed Mo micropillars as a function of pre-strain. J. Mater. Res. 2010:25:199-206.

[120] Barabash RI, Bei H, Gao YF, Ice GE. Interface strength in NiAl-Mo composites from 3D X-ray microdiffraction. Scripta Mater. 2011:64:900-903.

[121] Barabash RI, Bei H, Ice GE, Gao YF, Barabash OM 3D X-ray strain microscopy in twophase composites at submicron length scales. JOM 2011:63(3):30-34.

[122] Bei H, Barabash RI, Ice GE, Liu W, Tischler J, George EP. Spatially-resolved strain measurements in Mo-alloy micro-pillars by differential aperture X-ray microscopy. Appl. Phys. Lett. 2008:93:071904.

[123] Zimmermann J, van Petegem S, Grolimund D, Bei H, George EP, van Swygenhoven H. Effects of FIB milling and pre-straining on the microstructure of directionally solidified Mo pillars: a Laue diffraction analysis. Scripta Mater. 2010:62:746-749.

[124] Zimmermann J, van Swygenhoven H, Marichal C, van Petegem S, Borca C, Bartova B, Oveisi E, Herbert C. Slip in directionally solidified Mo-alloy micropillars - Part I: nominally dislocation-free pillars. Acta Mater. 2012:60:4604-4613.

[125] Zimmermann J, van Swygenhoven H, van Petegem S, Borca C. Slip in directionally solidified Mo-alloy micropillars - Part II: pillars containing defects. Acta Mater. 2012:60:4614-4622.

[126] Bei H, George EP, Brown DW, Pharr GM, Choo H, Porter WD, Bourke MAM. Thermalexpansion behavior of a directionally solidified NiAl-Mo composite investigated by neutron diffraction and dilatometry. J. Appl. Phys. 2005:97:123503.

[127] Johanns KE, Sedlmayr A, Sudharshan Phani P, Mönig R, Kraft O, George, EP, Pharr GM. In-situ tensile testing of single-crystal molybdenum-alloy fibers with various dislocation densities in a scanning electron microscope. J. Mater. Res. 2012:27:508-520.

[128] Kwon J, Bowers ML, Brandes MC, McCreary V, Robertson IM, Sudharshan Phani P, Bei H, Gao YF, Pharr GM, George EP, Mills MJ. Characterization of dislocation structures and deformation mechanisms in as-grown and deformed directionally solidified NiAl-Mo composites. Acta Mater. 2015:89:315-326.

[129] Rinadli A, Peralta P, Friesen C, Sieradzki K. Sample-size effects in the yield behavior of nanocrystalline nickel. Acta Mater. 2008:56:511.

[130] Wang W, Zhong Y, Lu K, Lu L, McDowell DL, Zhu T. Size effects and strength fluctuation in nanoscale plasticity. Acta Mater. 2012:60:3302.

[131] Ngan AHW, Zuo L, Wo PC. Size dependence and stochastic nature of yield strength of micron-sized crystals: a case study on $\mathrm{Ni}_{3}$ Al. Proc. R. Soc. A 2006:462:1661. 
[132] Rice JR, Thomson R. Ductile versus brittle behavior of crystals. Phil. Mag. 1973:29:7397.

[133] Yu HH, Shrotriya P, Gao YF, Kim KS. Micro-plasticity of surface steps under adhesive contact: Part I, surface yielding controlled by single-dislocation nucleation. J. Mech. Phys. Solids 2007:55:489-516.

[134] Gao YF, Yu HH, Kim KS. Micro-plasticity of surface steps under adhesive contact: Part II - Multiple-dislocation mediated contact loading." J. Mech. Phys. Solids 2008:56:27592772.

[135] Rice JR. Dislocation nucleation from a crack tip: an analysis based on the Peierls concept. J. Mech. Phys. Solids 1992:40:239-271.

[136] Li TL, Lee JH, Gao YF, Pharr GM, Huang M, Tsui TY. Geometric Effects on Dislocation Nucleation in Strained Electronics. Appl. Phys. Lett. 2009:94:17095.

[137] Gao YF. A Peierls perspective on mechanisms of atomic friction. J. Mech. Phys. Solids 2010:58:2023-2032.

[138] Lee JH, Gao YF. Mixed-mode singularity and temperature effects on dislocation nucleation in strained interconnects." Int. J. Solids Struct. 2011:48:1180-1190.

[139] Li TL, Lee JH, Gao YF. Finite element studies of homogeneous and heterogeneous dislocation nucleation based on the Rice-Peierls framework. Mater. Res. Soc. Symp. Proc. 2011:1363:RR03-01.

[140] Zhang JN, Lee JH, Lou J, Gao YF. Integration of contact size dependence and thermal activation in atomic friction. Extreme Mech. Lett. 2015:2:60-64.

[141] Zhu T, Li J, Yip S. Atomistic study of dislocation loop emission from a crack tip. Phys. Rev. Lett. 2004:93:025503.

[142] Ryu S, Kang K, Cai W. Predicting the dislocation nucleation rate as a function of temperature and stress. J. Mater. Res. 2011:26:2335-2354.

[143] Chen W, Cui P, Zhu WG, Kaxiras E, Gao YF, Zhang ZY. Atomistic mechanisms for bilayer growth of graphene on metal substrates. Phys. Rev. B 2015:91:045408.

[144] Packard CE, Franke O, Homer ER, Schuh CA. Nanoscale strength distribution in amorphous versus crystalline metals. J. Mater. Res. 2010:25:2251-2263.

[145] Huang SY, Gao YF, An K, Zheng LL, Wu W, Teng ZK, Liaw PK. Deformation mechanisms in a precipitation-strengthened ferritic superalloy revealed by in situ neutron diffraction studies at elevated temperatures. Acta Mater. 2015:83:137-148.

[146] Maaß R, Meza L, Gan B, Tin S, Greer JR. Ultrahigh strength of dislocation-free $\mathrm{Ni}_{3} \mathrm{Al}$ nanocubes. Small 2012:8:1869.

[147] Parthasarathy TA, Rao SI, Dimiduk DM, Uchic MD, Trinkle DR. Contributions to size effect of yield strength from the stochastics of dislocation source lengths in finite samples. Scr. Mater. 2007:56:313-316.

[148] Morris JR, Bei H, Pharr GM, George EP. Size effects and stochastic behavior of nanoindentation pop in. Phys. Rev. Lett. 2011:106:165502.

[149] Sudharshan Phani P, Johanns KE, George EP, Pharr GM. A simple stochastic model for yielding in specimens with limited number of dislocations. Acta Mater. 2013:61:24892499.

[150] Sudharshan Phani P, Johanns KE, George EP, Pharr GM. A stochastic model for the size dependence of spherical indentation pop-in. J. Mater. Res. 2013:28:2728-2739.

[151] Gagel J, Weygand D, Gumbsch P. Formation of extended prismatic dislocation structures under indentation. Acta Mater. 2016:111:399. 
[152] Ngan AHW, Ng KS. Transition from deterministic to stochastic deformation. Phil. Mag. 2010:90:1937.

[153] Gu R, Ngan AHW. Dislocation arrangement in small crystal volumes determines powerlaw size dependence of yield strength. J. Mech. Phys. Solids 2013:61:1531.

[154] Bei H, Shim S, Miller MK, Pharr GM, George EP. Effects of focused ion beam milling on the nanomechanical behavior of a molybdenum-alloy single crystal," Appl. Phys. Lett. 2007:91:111915.

[155] Shim S, Bei H, Miller MK, Pharr GM, George EP. Effects of focused ion beam milling on the compressive behavior of directionally solidified micropillars and the nanoindentation response of an electropolished surface. Acta Mater. 2009:57:503-510.

[156] Greer JR, Espinosa H, Ramesh KT, Nadgorny E. Comment on "Effects of focused ion beam milling on the nanomechanical behavior of a molybdenum-alloy single crystal" Appl. Phys. Lett. 91111915 (2007). Appl. Phys. Lett., 2008:92:096101.

[157] Wang Z, Bei H, George EP, Pharr GM. Influences of surface preparation on nanoindentation pop-in in single-crystal Mo. Scripta Mater. 2011:65:469-472.

[158] Brenner SS. Tensile strength of whiskers. J. Appl. Phys. 1956:27:1484-1491.

[159] Brenner SS. Plastic deformation of copper and silver whiskers. J. Appl. Phys. 1957:28:1023-1026.

[160] Tian L, Cheng YQ, Shan ZW, Li J, Wang CC, Han XD, Sun J, Ma E. Approaching the ideal elastic limit of metallic glasses. Nature Coтmu. 2012:3:609.

[161] Richter G, Hillerich K, Gianola DS, Mönig R, Kraft O, Volkert CA. Ultrahigh strength single crystalline nanowhiskers grown by physical vapor deposition. Nano Lett. 2009:9:3048-3052.

[162] Lowry MB, Kiener D, LeBlanc MM, Chisholm C, Florando JN, Morris Jr JW, Minor AM. Achieving the ideal strength in annealed molybdenum nanopillars. Acta Mater. 2010:58:5160-5167.

[163] Magagnosc DJ, Kumar G, Schroers J, Felfer P, Cairney JM, Gianola DS. Effect of ion irradiation on tensile ductility, strength and fictive temperature in metallic glass nanowires. Acta Mater. 2014:74:165-182.

[164] Yang Y, Ye J, Lu J, Wang Q, Liaw PK. Revelation of the effect of structural heterogeneity on microelasticity in bulk metallic-glasses. J. Mater. Res. 2010:25:563575.

[165] Choi IC, Zhao Y, Kim YJ, Yoo BG, Suh JY, Ramamurty U, Jang JI. Indentation size effect and shear transformation zone size in a bulk metallic glass in two different structural states. Acta Mater. 2012:60:6862-6868.

[166] Choi IC, Zhao Y, Yoo BG, Kim YJ, Suh JY, Ramamurty U, Jang JI. Estimation of the shear transformation zone size in a bulk metallic glass through statistical analysis of the first pop-in stresses during spherical nanoindentation. Scritpta Mater. 2012:66:923-926.

[167] Fan Y, Iwashita T, Egami T. How thermally activated deformation starts in metallic glass. Nature Comm. 2014:5:5083.

[168] Fan Y, Iwashita T, Egami T. Crossover from localized to cascade relaxation in metallic glasses. Phys. Rev. Lett. 2015:115:045501. 
Tables

Table I Fitting parameters for the nanoindentation pop-in results in Figs. 3 and 4, for NiAl and Mo single crystals and $\mathrm{Zr}_{52.5} \mathrm{Al}_{10} \mathrm{Ti}_{5} \mathrm{Cu}_{17.9} \mathrm{Ni}_{14.6}$ metallic glass. For Mo single crystals at different pre-strain levels, dislocation densities have been measured by the polychromatic x-ray micro-diffraction (PXM) technique, conducted at Advanced Photon Source.

\begin{tabular}{|c|c|c|c|c|c|c|}
\hline Samples & $\begin{array}{l}\mathrm{NiAl} \text { (various } \\
\text { orientations) }\end{array}$ & $\begin{array}{c}\text { As-grown Mo } \\
<001>\end{array}$ & $\begin{array}{c}1.5 \% \text { pre- } \\
\text { strained Mo } \\
<001>\end{array}$ & $\begin{array}{l}5.0 \% \text { pre- } \\
\text { strain Mo } \\
\quad<100>\end{array}$ & $\begin{array}{c}\text { Zr-based } \\
\text { BMG (as-cast) }\end{array}$ & $\begin{array}{c}\text { Zr-based } \\
\text { BMG (fully } \\
\text { annealed) }\end{array}$ \\
\hline$\tau_{\text {defect }}$ & $0.02 \tau_{\text {th }}$ & $0.04 \tau_{\text {th }}$ & $0.046 \tau_{\text {th }}$ & $0.048 \tau_{\text {th }}$ & $0.98 \mathrm{GPa}$ & $0.98 \mathrm{GPa}$ \\
\hline$\rho_{\text {defect }}\left(\mu \mathrm{m}^{-3}\right)$ & 0.372 & 0.0053 & 17.5 & 55 & 0.0047 & 0.00012 \\
\hline $\begin{array}{l}\text { Defect spacing } \\
\qquad(\mu \mathrm{m})\end{array}$ & 1.39 & 5.70 & 0.38 & 0.26 & 5.97 & 20.3 \\
\hline $\begin{array}{c}\text { Dislocation } \\
\text { density }\left(\mu \mathrm{m}^{-2}\right) \\
(\mathrm{PXM})\end{array}$ & -- & 0.1047 & 534.9 & 1599.0 & -- & -- \\
\hline $\begin{array}{c}\text { Dislocation } \\
\text { spacing }(\mu \mathrm{m}) \\
(\mathrm{PXM})\end{array}$ & -- & 3.09 & 0.0432 & 0.0250 & -- & -- \\
\hline
\end{tabular}




\section{Figure Captions}

Figure 1 (a) Representative micro-pillar compression tests usually focus on the scaling relationship, especially the slope in the log-log plot, between the normal yield or flow stress and the pillar diameter. Data compilation includes FCC single crystals: $\mathrm{Ni}, \mathrm{Au}$, $\mathrm{Cu}$, and $\mathrm{Al}$; $\mathrm{BCC}$ single crystals: $\mathrm{V}$ and $\mathrm{Mo}$; discrete dislocation plasticity simulations; and metallic glasses. Because of sample preparation, these micro-pillars are usually of $\sim 5: 1$ length-to-diameter ratio. (b) In contrast to the scaling exponent study in Fig. 1(a), nanoindentation pop-in tests on Mo <001> single crystals find a totally different relationship between the pop-in stress and the indenter radius, including a transition between two asymptotes in Li et al. [36]. Similar trends are also found for an as-cast Zr-based bulk metallic glass sample in Bei et al. [51]. (c) In contrast to the scaling exponent study in Fig. 1(a), compression tests on as-grown Mo alloy fibers have virtually no pre-existing defects, and there is no size effect. The prestrained samples, however, show different degrees of strength statistics that give rise to various scaling exponents in Bei et al. [40]. The pillars under compression tests are usually of 2:1 3:1 length-to-diameter ratio. Long fibers under tension show dramatic scatter of the strength in Chisholm et al. [45] and Loya et al. [46]. These tensile samples span a range of 3-10 $\mu \mathrm{m}$ in length. (d) Among the micro-pillar tests complied here for metallic glasses, data from Bharathula and Flores [65] resemble the trends in the intermediate-to-large indenters in Fig. 1(b) and in the 4\% pre-strained samples under compression in Fig. 1(c).

Figure 2 Pop-in corresponds to the sudden displacement excursions on the load-displacement $(P-h)$ curves using spherical indenters. (a) Mo (100) single crystal with indenter radius $R=220 \mathrm{~nm}$. (b) Ni (100) with $R=220 \mathrm{~nm}$. (c) Ni (100) with $R=580 \mathrm{~nm}$. Data compiled from Xia et al. [72]. If the pop-in excursions are removed, the resulting consolidated curves again display elastic contact response, although the effective indenter radius should increase.

Figure 3 The pop-in stress, $\tau_{R S S}^{\max }$, is the maximum resolved shear stress among all the slip systems under the contact. The statistical measurements give rise to the cumulative probability, meaning that provided with a strength value, there is a corresponding probability for the pop-in to take place. Solids curves are theoretical predictions, and 
discrete symbols are experimental results: (a) Mo <001> single crystals indented with spherical indenters with various radii in Li et al. [36], which correspond to the data presented in Fig. 1(b), and (b) NiAl single crystals with one spherical indenter but with varying crystallographic orientations in Li et al. [81].

Figure 4 Nanoindentation pop-in tests on a Zr-based metallic glass with three different indenter radii in Li et al. [57]. The as-cast samples were annealed at different temperatures for the same annealing time. These cumulative probability measurements qualitatively resemble results in Fig. 3(a), i.e., approaching the ideal strength for the rightmost curves and approaching the defect strength for the leftmost curves.

Figure 5 The upper bound values of the nanoindentation pop-in stress and the pillar yield stress are compared here. (a) For Mo single crystals under small stressed volumes, the popin stress, $\tau_{R S S}^{\max }$, reaches the theoretical shear strength of about $15.7 \mathrm{GPa}$. Refer also to the upper asymptote in Fig. 1(b). (b) For Mo alloy fibers under compression, the upper bound value is about $10 \mathrm{GPa}$ (refer also to the upper bound in Fig. 1(c)), which is converted to a shear strength of about $4.6 \mathrm{GPa}$, or about $1 / 3$ of the theoretical shear strength. The discrepancy between the upper bound values for these two types of tests arises from surface nucleation of dislocations, as explained in details in the text. (c) To exclude the alloying effect since the compositions in (a) and (b) are slightly different, the pop-in test was conducted on the Mo alloy fiber, leading again to a popin stress that is very close to the theoretical shear strength. Data compiled from Bei et al. [91].

Figure 6 Micro-pillar compression tests of as-grown and pre-strained Mo alloy fibers, as representative examples for the data points in Fig. 1(c) [39,40]. (a) The as-grown samples with edge width of about $500 \mathrm{~nm}$. (b) The $4 \%$ pre-strained samples with edge width of about $1200 \mathrm{~nm}$. (c) The 4\% pre-strained samples with edge width of about $550 \mathrm{~nm}$. Deformed shapes are also included in the SEM images as insets. (d) Yield stress plotted against the pillar size.

Figure 7 In situ TEM tests of Mo alloy fibers in Chisholm et al. [45]. (a) For as-grown Mo alloy fibers without dislocations, TEM images are presented before and after test, where the lines in the image before the test are bend contours. The engineering stress 
reaches a maximum value of about $7 \mathrm{GPa}$, which is lower than the $9.8 \mathrm{GPa}$ strength in short-pillar tests in Fig. 6(a). (b) Mo alloy fibers with pre-existing dislocations, with local dislocation microstructures analyzed in the gauge window, starting from initial state, to the activation of spiral arm source at $1.5 \mathrm{GPa}$, and to further evolutions to a flow stress of about $3 \mathrm{GPa}$.

Figure 8 From extensive statistical measurements by nanoindentation pop-in (e.g., Fig. 1(b), Fig. 3, and Fig. 4) and by as-grown and pre-strained pillars (e.g., Fig. 1(c), Fig. 6 and Fig. 7), it is anticipated that the material strength (as normalized by the shear modulus) follows a master plot with respect to the stressed volume size (as normalized by the pre-existing defect density, also involving the Schmid factor that describes the crystallographic orientation effects). States A and D are two asymptotes, corresponding to thermally activated, homogeneous dislocation nucleation and bulk yield stress, respectively. State B shows the convolution between thermal activation and the stochastic nature of finding the heterogeneous dislocation nucleation sources, while Stage $\mathrm{C}$ is dominated by the spatial stochasticity. The asymptotes can be varied by a number of controlling parameters. Note that the variation of the bulk asymptote is exaggerated; any bulk flow stress change will not be easily noticed on this plot since there is at least one order-of-magnitude difference between the theoretical stress and bulk flow stress.

Figure 9 The thermal activation model may incorrectly interpret the pop-in data when there is a large "tail" on the cumulative probability curve in Li et al. [81]. (a) For NiAl indentations in Fig. 3(b), cumulative probability for <207> orientation is converted to probability, which clearly shows two Gaussian peaks. (b) The thermal activation model should be only applied to the right Gaussian peak, giving rise to the dashed curve, while an incorrect use of the thermal activation model for the upper portion of the cumulative probability curve gives the solid curve. Fitting by using the unified model presented in Fig. 10 has been given in Fig. 3(b).

Figure 10 The stochastic model is essentially to determine the probability of finding the weakest link, being the heterogeneous dislocation nucleation sources in our model here. For (a) nanoindentation pop-in and (b) uniaxial pillar tests (tension or compression), the two asymptotes are deterministic while the intermediate stage is stochastic. It should 
be noted that the dislocation density, $\rho_{\perp}$, differs from the density of the heterogeneous dislocation nucleation sources, $\rho_{\text {defect }}$. Synchrotron x-ray diffraction studies [37] have shown that the defect spacing is much larger than the dislocation spacing, as shown in Table 1.

Figure 11 Investigations on the role played by FIB-induced surface damages on nanoindentation pop-ins in Bei et al. [154] and Shim et al. [155]. (a) Schematic illustration of the FIB milling with two incident angles, together with the subsequent statistical pop-in measurements. (b) Out of three surface preparation conditions, the as-grown surface shows a clear pop-in at high pop-in stress, while the FIB-milled surfaces show very low pop-in stresses or no visible pop-ins. (c) For the FIB milling with normal incidence, the load-displacement curves are affected by the applied voltage.

Figure 12 Investigations on the role played by FIB-induced surface damages on micro-pillar compression tests in Shim et al. [155]. (a) FIB milling is conducted on one as-grown Mo alloy fiber (by directional solidification), so as to make a cylindrical fiber that mimics the procedure typically employed in literature (e.g., Fig. 1(a)). (b) Comparisons between the FIB-milled pillars $(\sim 670 \mathrm{~nm})$ and directionally solidified pillars ( $\sim 550 \mathrm{~nm}$, pre-strained at different levels). (c) Comparisons between the FIBmilled Mo alloy pillars ( $\sim 670 \mathrm{~nm}$ ) and the FIB-milled pure Mo <100> pillars in Brinckmann et al. [22]. (d) A schematic illustration of the FIB-induced damages.

Figure 13 Investigations on the role played by surface polishing conditions on nanoindentation pop-ins [157]. (a) Nanoindentation pop-in tests on Mo <100> single crystals with various polishing conditions. (b) The corresponding cumulative pop-in probability curves. The electropolished sample has the least damage.

Figure 14 Investigations on the role played by pre-straining on nanoindentation pop-ins in Bei et al. [37]. (a) The cumulative pop-in probability versus the indenter radius for annealed and pre-strained Mo single crystals. (b)-(d) are the corresponding cumulative probability versus pop-in stress curves for the annealed, $1.5 \%$ pre-strained, and 5\% pre-strained samples, respectively. Legend denotes the radius (in $\mu \mathrm{m}$ ) of the spherical indenters.

Figure 15 Re-plot of the cumulative pop-in probability curves in Fig. 14 with respect to the normalized indenter radius in Bei et al. [37]. As shown in the inset, the pre-straining 
does lead to about $10 \%$ increase of the bulk flow stress, which however is negligibly small compared to about $2,000 \%$ increase from the bulk strength to the theoretical strength. All data for annealed and pre-strained samples fall onto the master plot with $10 \%$ and $90 \%$ probability contour curves denoted as solid and dashed curves, respectively.

Figure 16 Investigations on the role played by the loading rate on fiber tensile tests in Loya et al. [46]. (a) In situ SEM test of long Mo alloy fibers (prepared by directional solidification, annealing or pre-straining, and subsequently isolating Mo alloy fibers from the composite by etching away the NiAl matrix). (b) Measured and predicted yield strengths, plotted against the product of the specimen length and the 1D defect density.

Figure 17 Pillar compression and tension results complied from Bei et al. [39,40], Johanns et al. [127], Chisholm et al. [45], Sudharshan Phani et al. [44,149,150], and Loya et al. [46]. All materials are Mo alloys fibers made from directional solidification, and then annealed or pre-strained. (a) Strength versus the sample length. (b) Strength versus the normalized length by the 1D defect density. These plots of strength versus length resemble the plot of strength versus width/diameter in Fig. 1(c) and the master plot in Fig. 15.

Figure 18 Whisker test results of $\mathrm{Fe}<111>, \mathrm{Cu}<111>$, and $\mathrm{Ag}<100\rangle$ single crystals in Brenner $[158,159]$ superimposed with the pillar/fiber tests of directionally solidified Mo alloy single crystal in Bei et al. [40], Zr-based BMG in Bharathula and Flores [65], $\mathrm{Cu}_{49} \mathrm{Zr}_{51}$ metallic glass in Tian et al. [160], and $\mathrm{Cu}<110>$ made by physical vapor deposition in Richter et al. [161]. Brenner's whiskers are typically of $\sim 4 \mathrm{~mm}$ in length. The theoretical strength, $\sigma_{t h}$, in the normal direction is calculated from the theoretical shear strength, $\tau_{t h}$, as obtained from first-principles calculations by Ogata et al. [38], and the Schmid factor. The upper-bound strength of fiber/wire tests is taken as $\sigma_{\max }^{\text {pillar }} \approx \sigma_{t h} / 3$. Schematic illustration of the $10 \%$ and $90 \%$ cumulative probability contour curves (as those in Fig. 8, and also shaded with different colors) is presented for these results. 
Figure 19 (a) For a Zr-based BMG, the nanoindentation pop-in stresses vary with respect to the indenter radius, similar to the tests on Mo single crystals but with very small difference between the two asymptotes in Bei et al. [51]. (b) The fracture energy measurements from three-point notch bending tests exhibit the transition from ductile to brittle behavior. (c) The defect density is found to vary with respect to the annealing condition. Defect density is determined from the nanoindentation pop-in measurements in Figs. 4 and 19(a) here and the unified model presented in Fig. 9(a). (d) The total fracture energy density (obtained from three-point notch bending test) is found to correlate to the defect density in an exponential manner in Li et al. [53]. Plot like this provides a structure-property relationship for metallic glasses.

Figure 20 (a) Schematic illustration of the nanoindentation pop-in statistics in BMG [57]. (b) Schematic illustration of the role played by the weak zones or defects near a crack tip [53]. For fully annealed sample, the structure approaches that of ideal glass so that the pop-in stress reaches theoretical strength, and correspondingly the fracture mode is cleavage, brittle crack. With the presence of many weak zones, the indentation response is metal like, and these weak zones form an effective process zone to shield the stress singularity near a crack, thus leading to a ductile fracture mode.

Figure 21 Atomistic simulations by Fan et al. [167,168] provide critical insights on the "weak zones" hypothesized in Fig. 20. For two systems (less stable in sys-I and more stable in sys-II) glasses, the number densities of individual thermally activated events are distinctively different. 
(a)

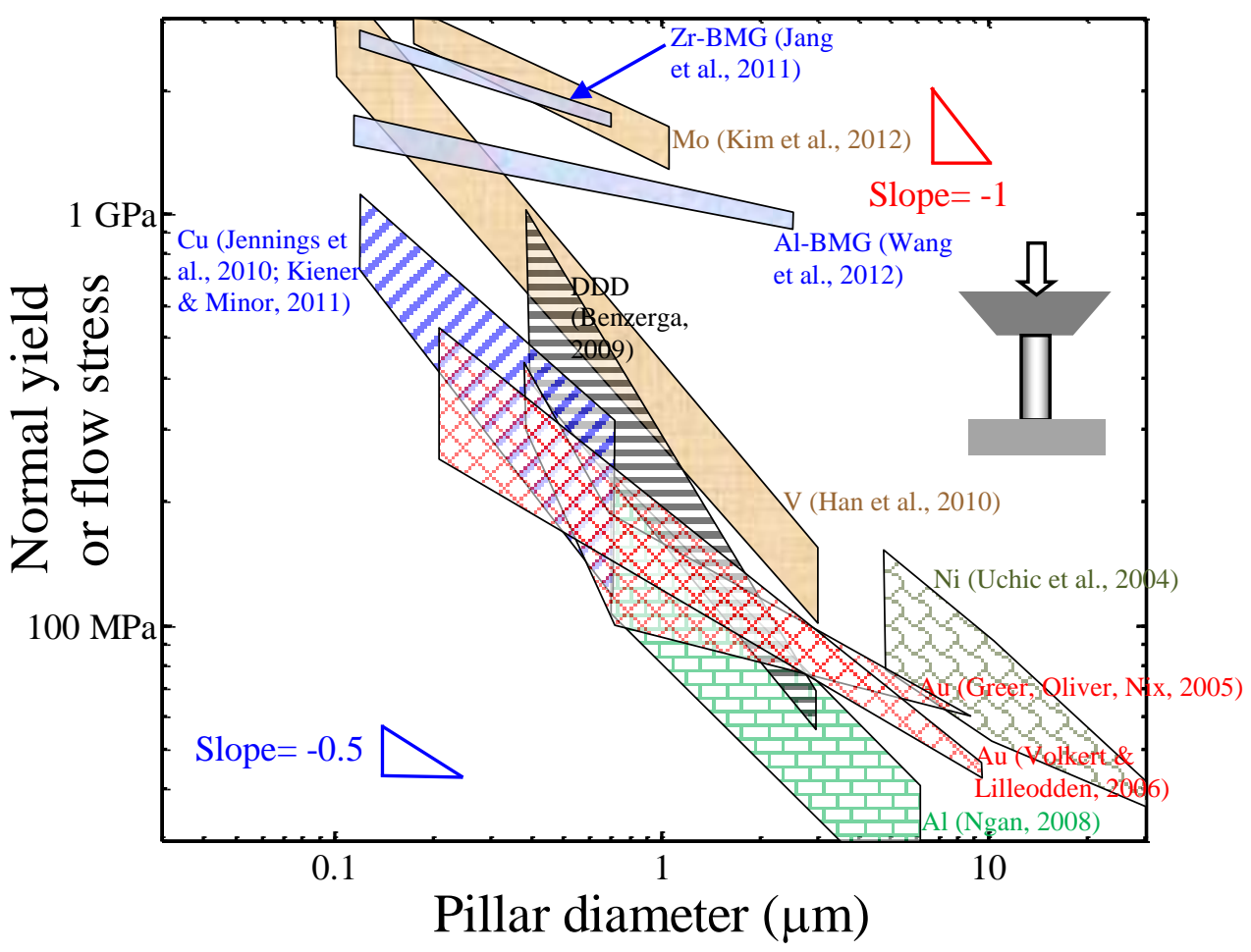

(b)

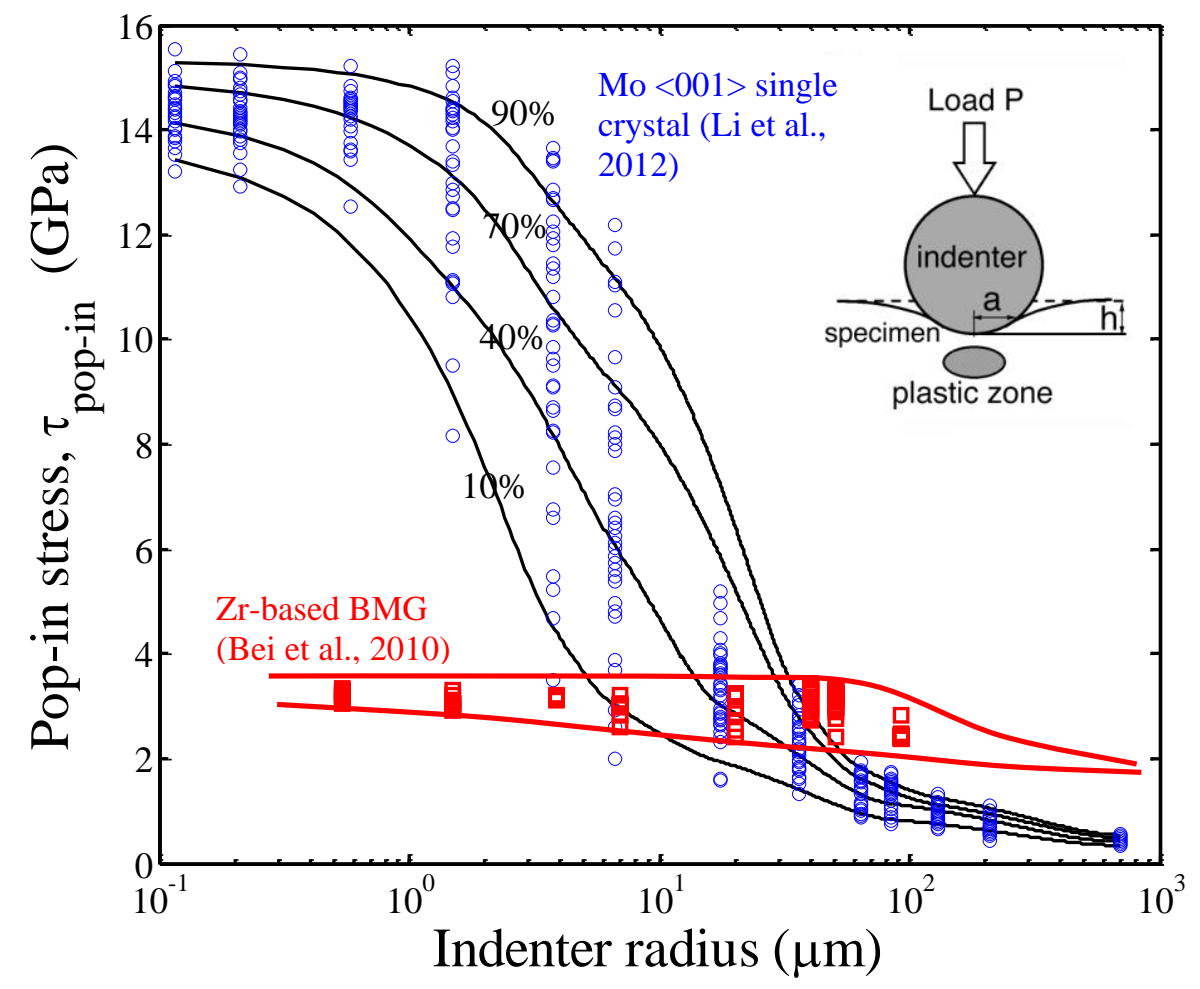

Figure 1 
(c)

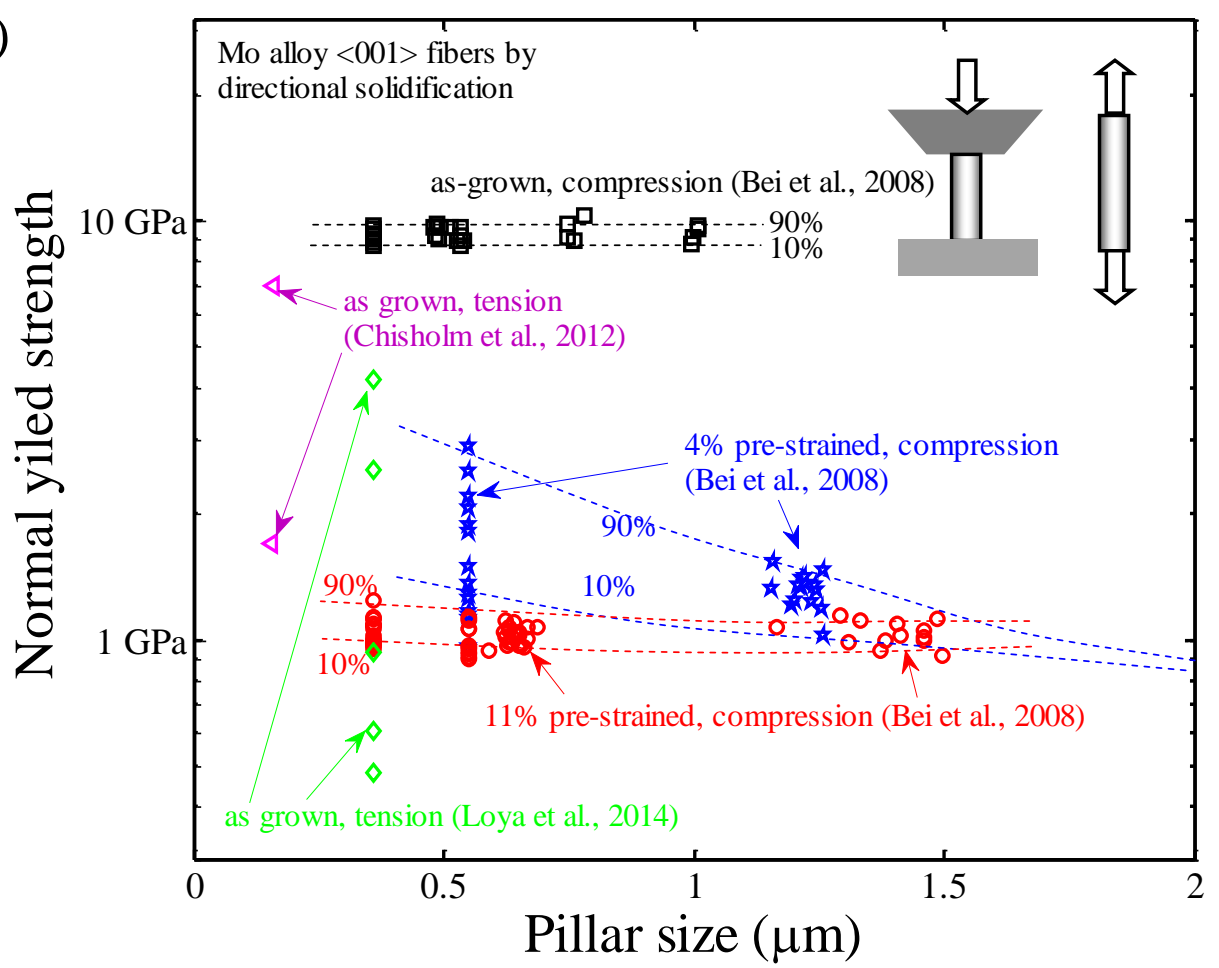

(d)

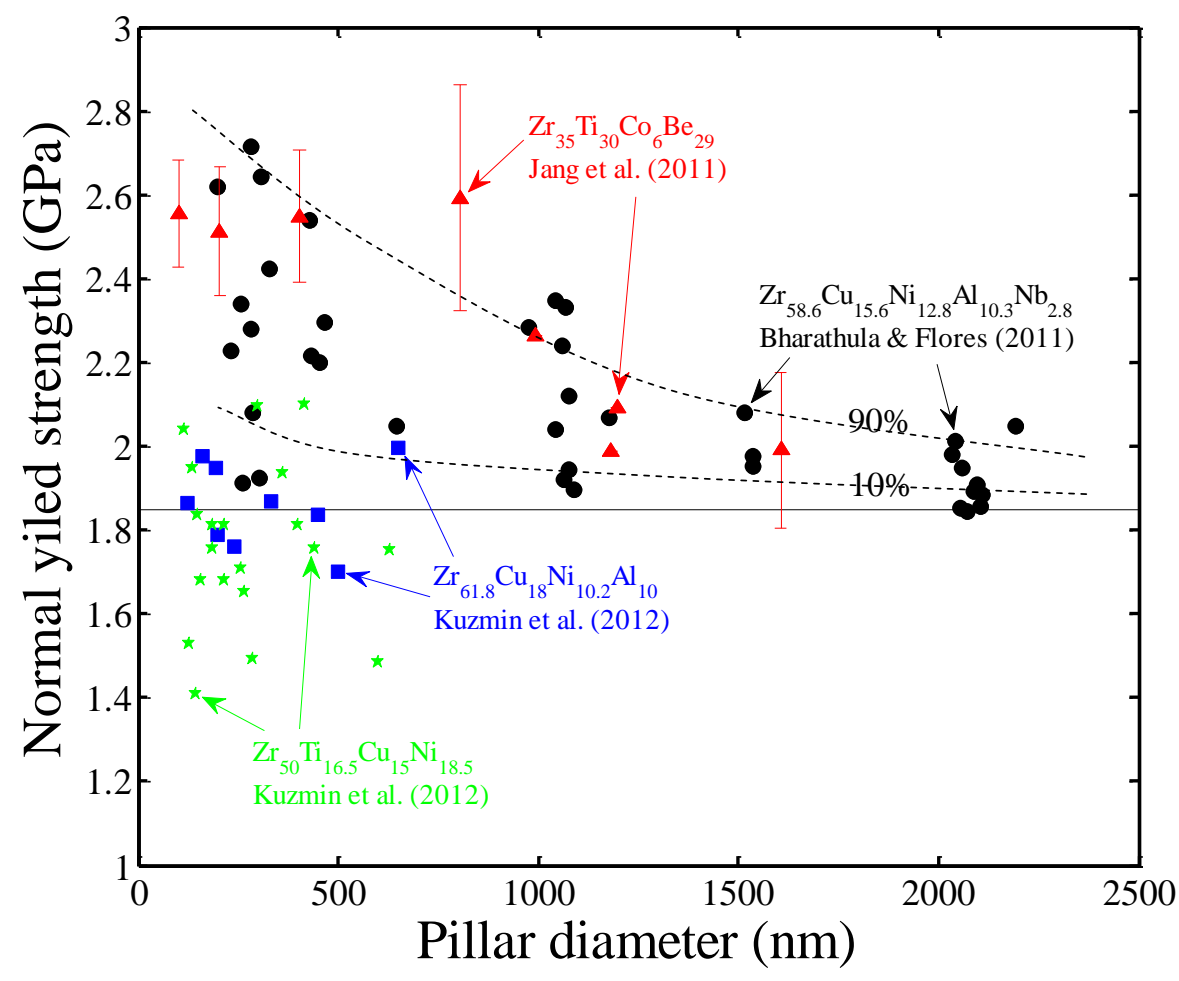

Figure 1 (cont'd) 
(a)

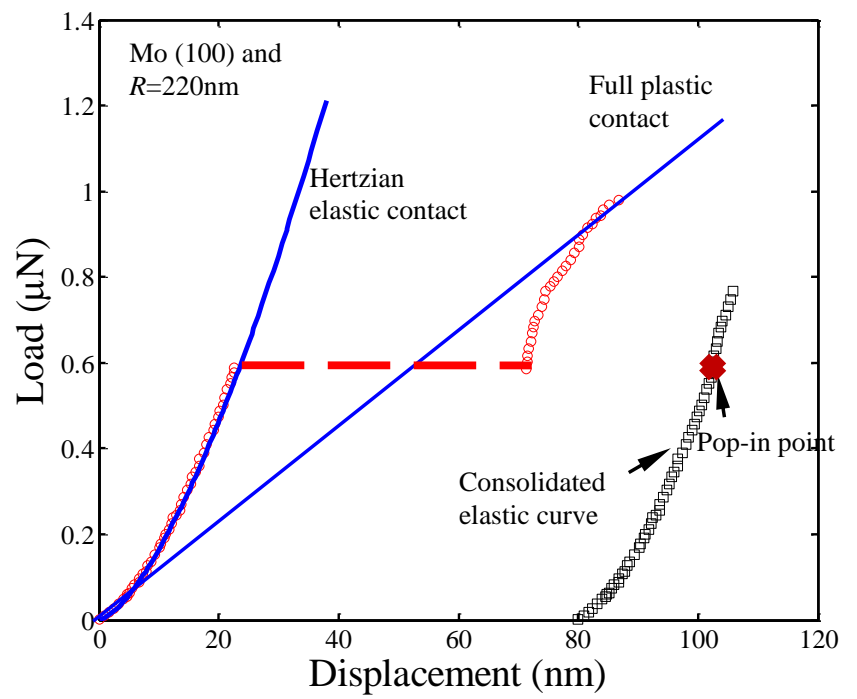

(b)

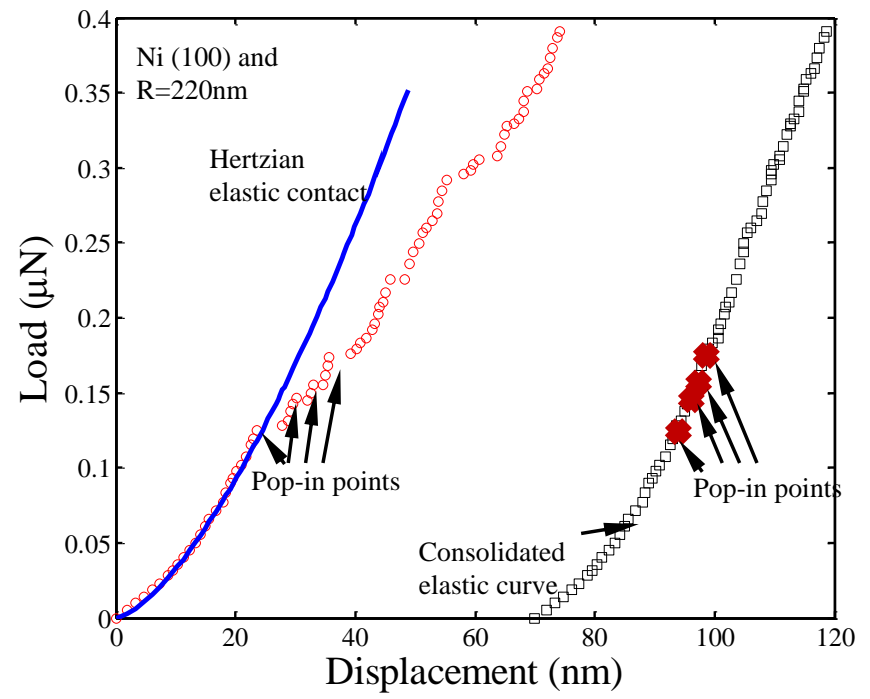

(c)

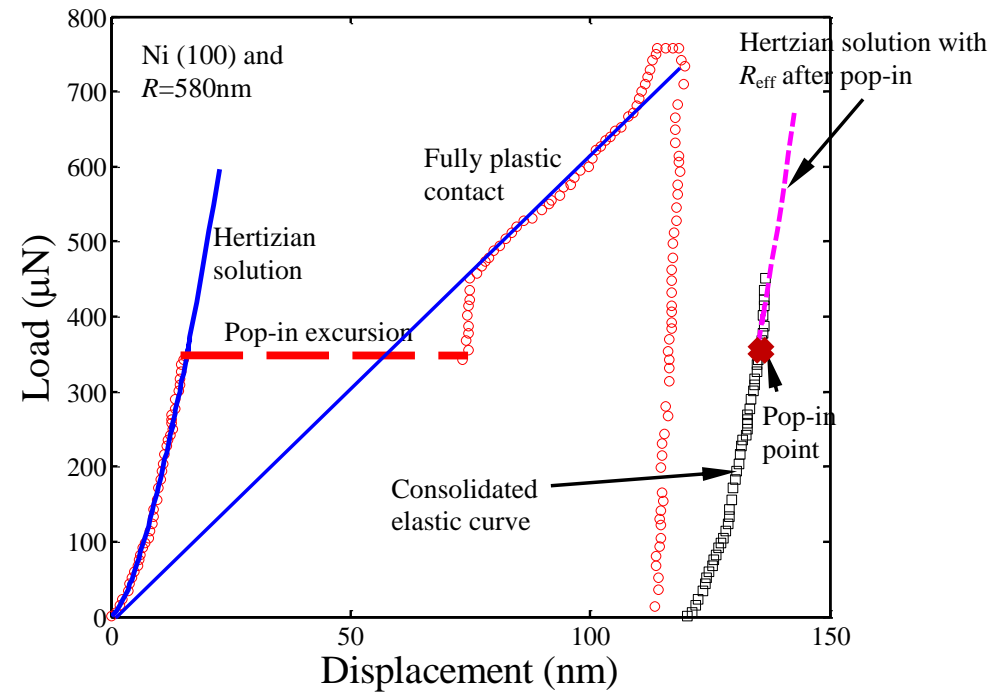

Figure 2 
(a)
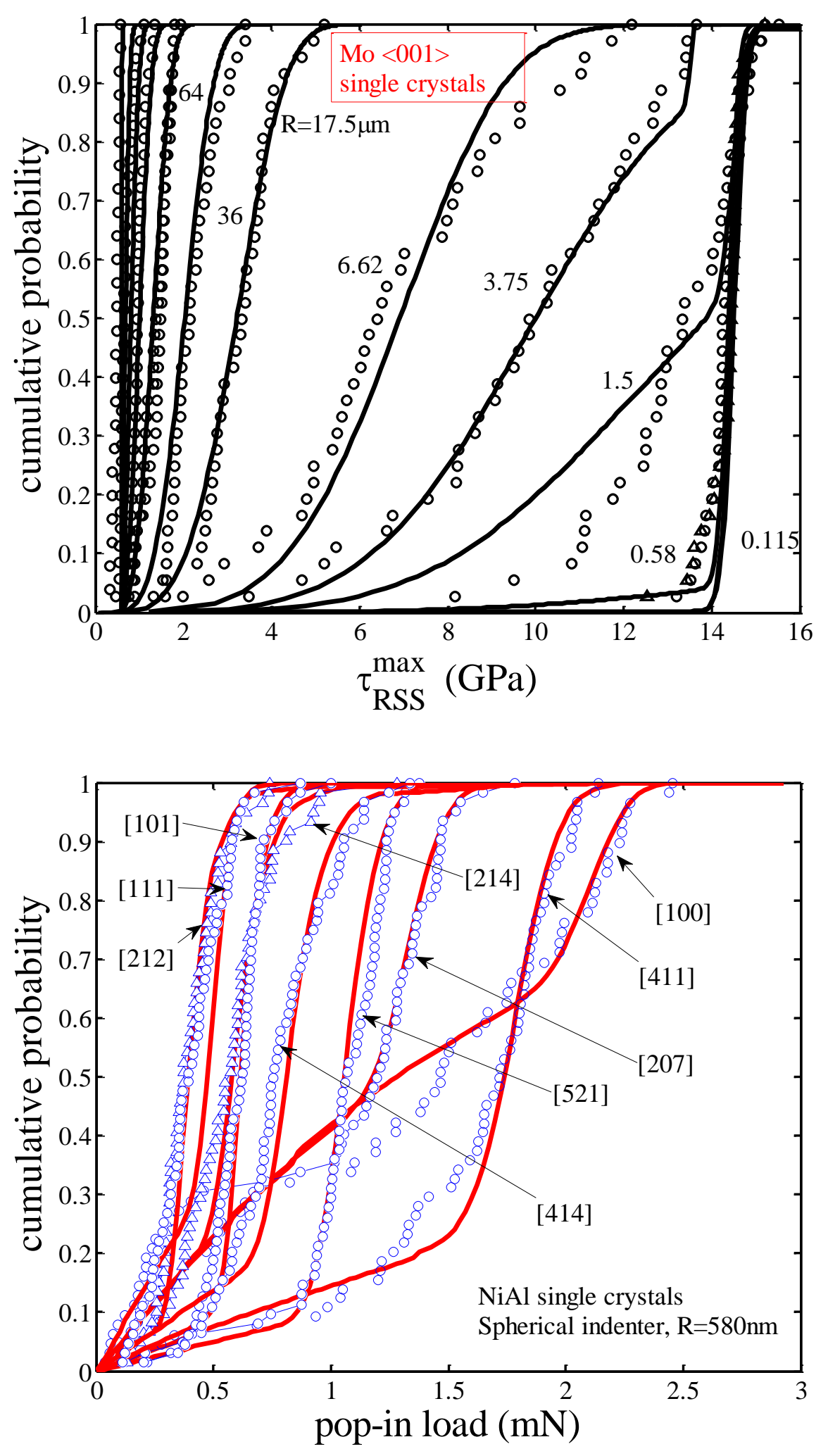

(b)

Figure 3 

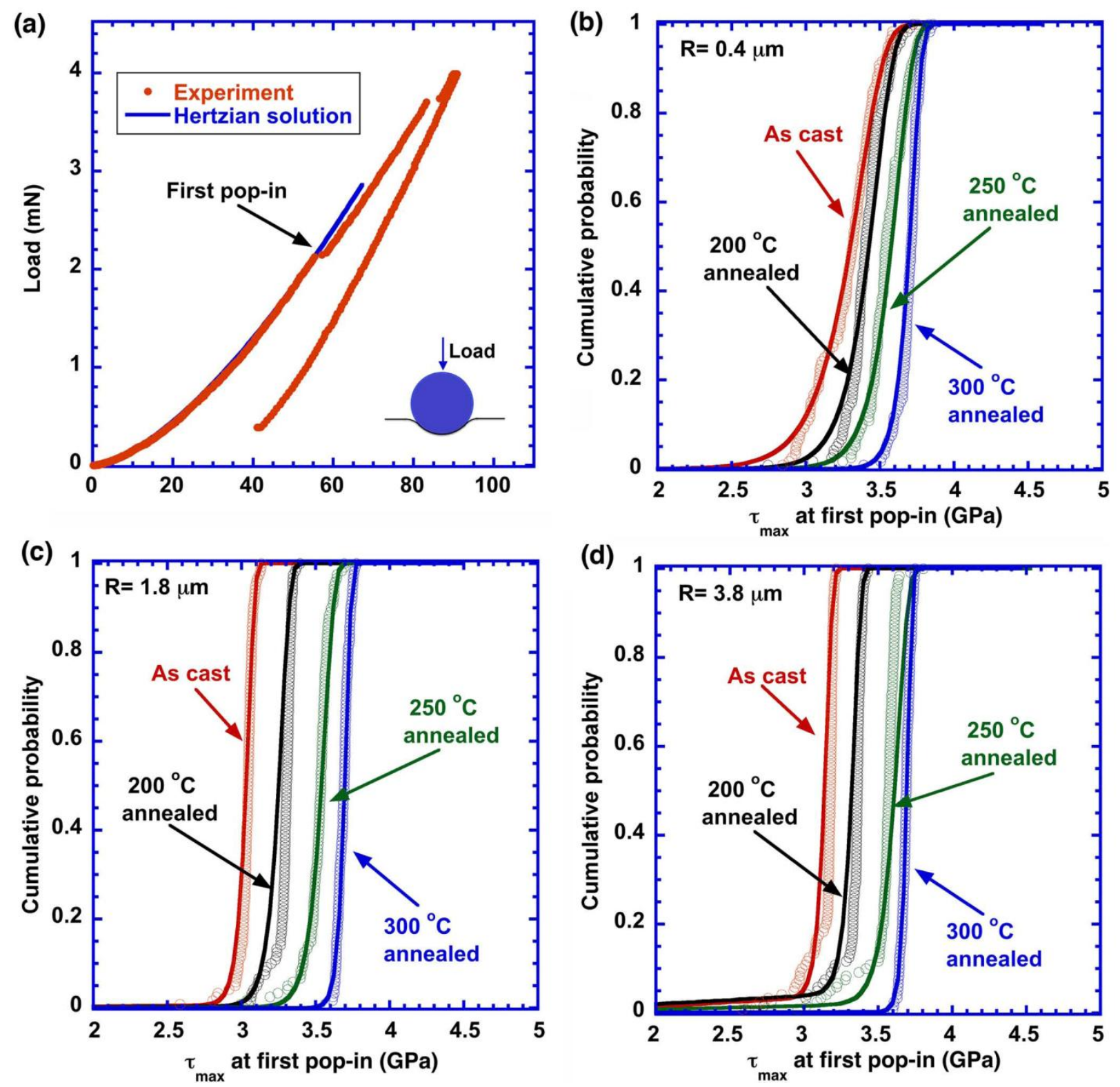

Figure 4 

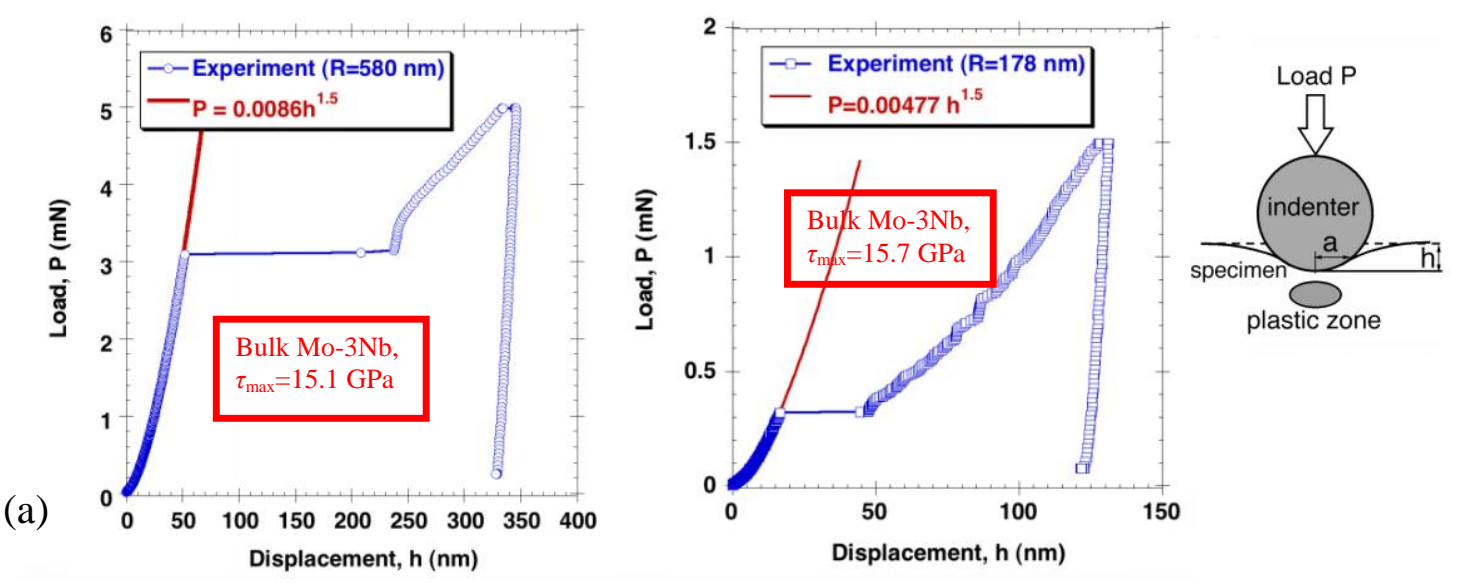

(b)
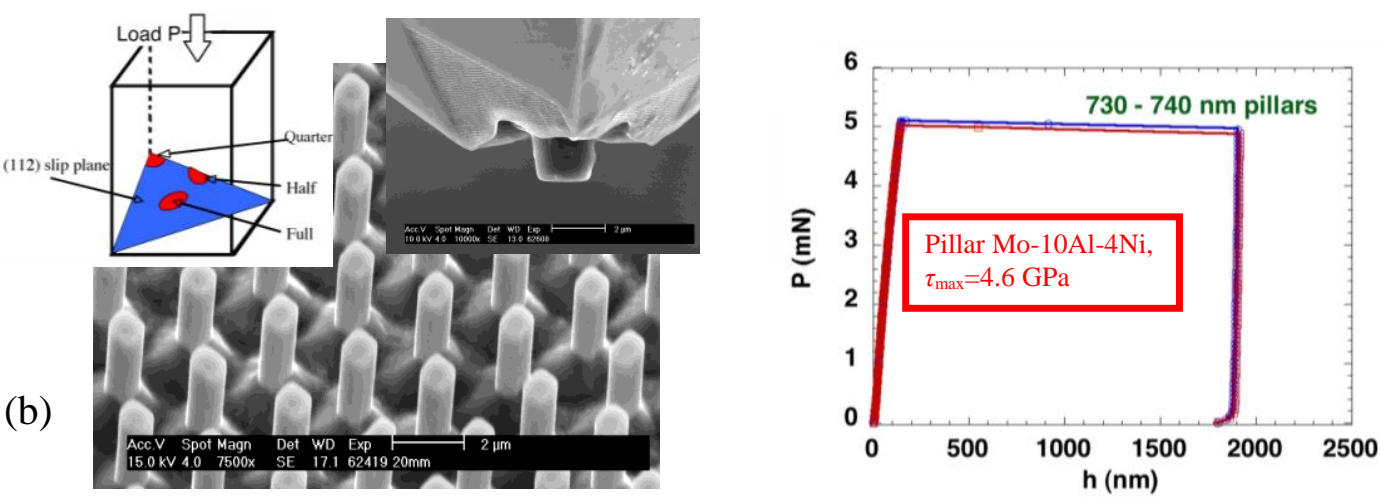

(c)
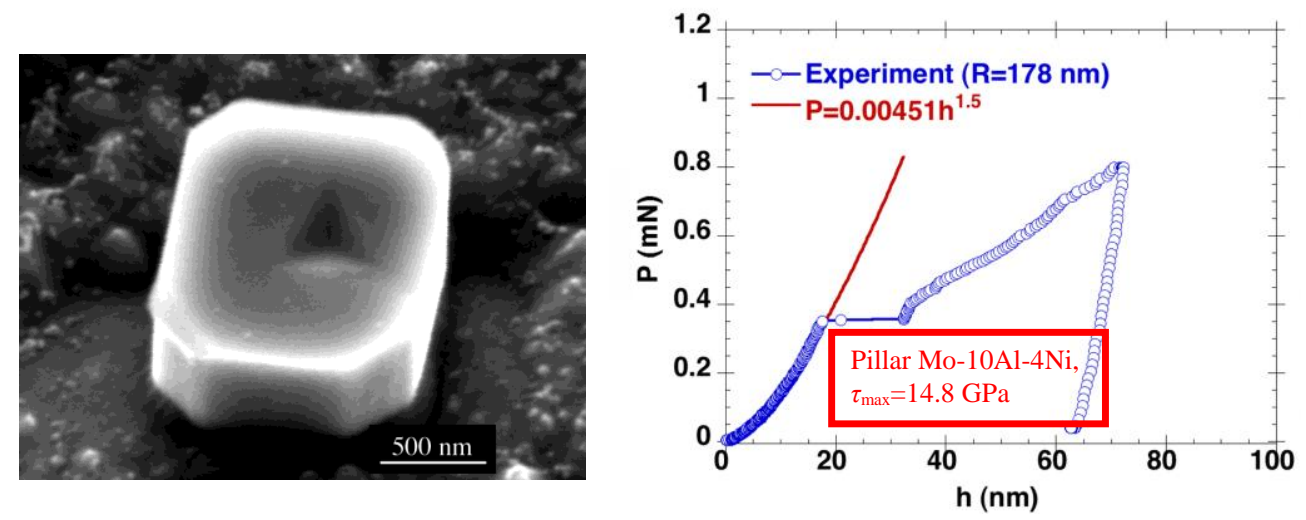

Figure 5 

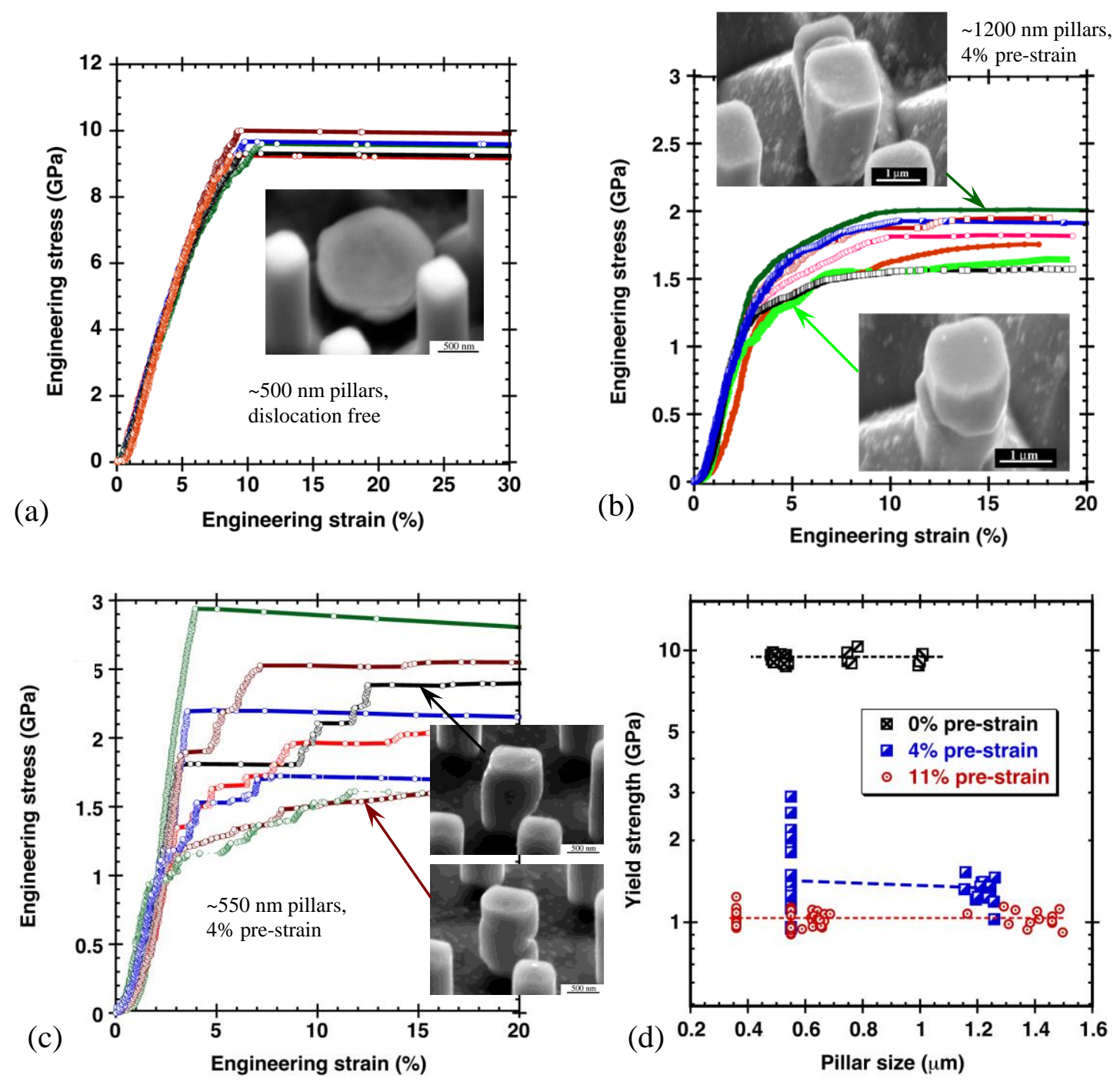

Figure 6 


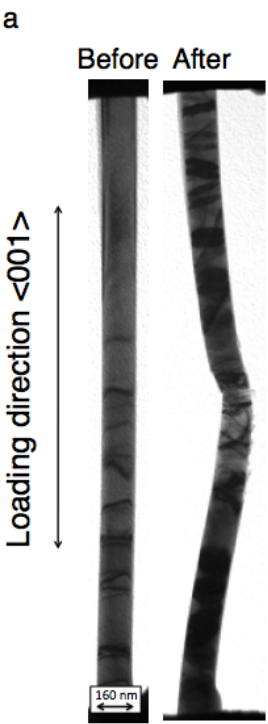

b

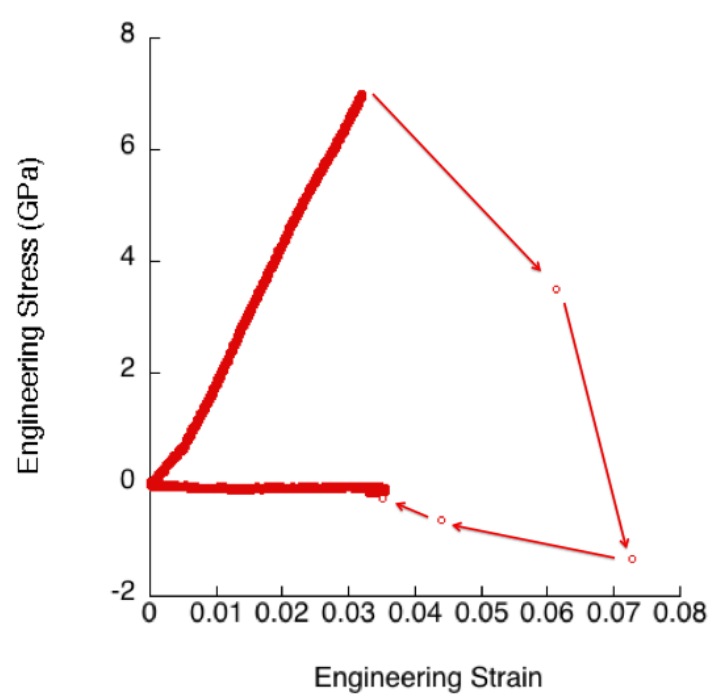

(a)

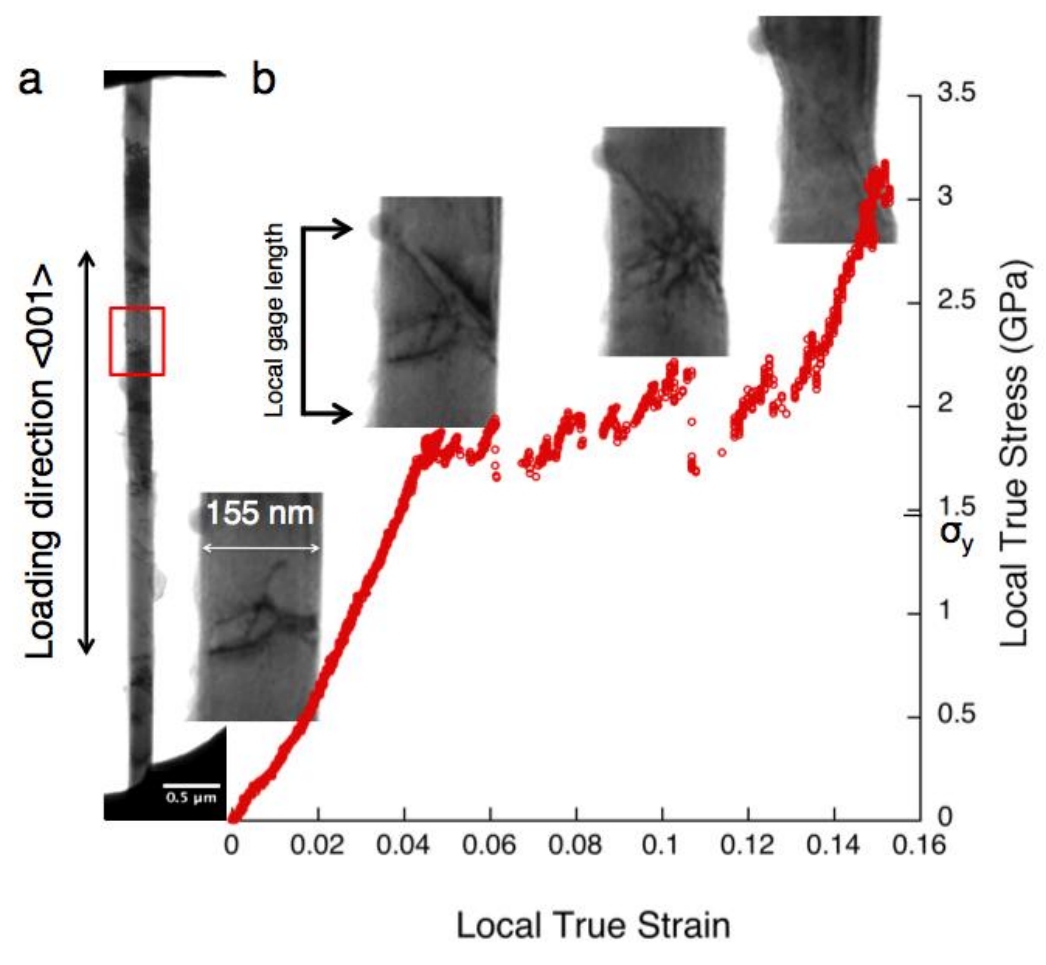

Figure 7 
Stress-assisted, thermally activated processes for homogeneous

dislocation nucleation

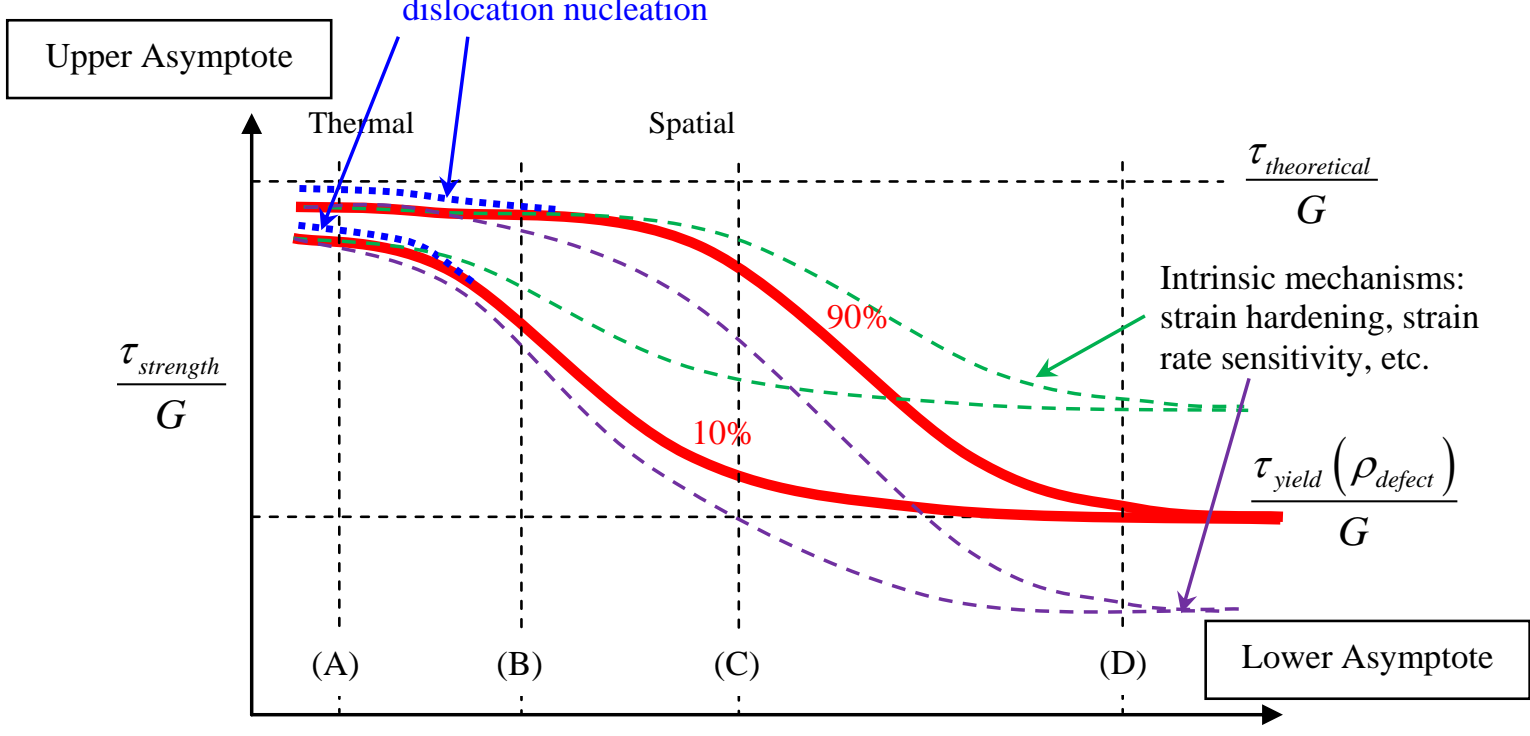

(Stressed Volume Size $) \times \sqrt[d]{\rho_{\text {defect }}} /($ Schmid Factor $)$

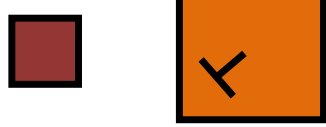

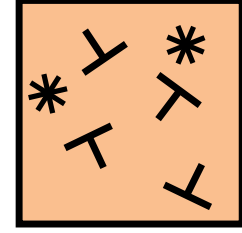

C

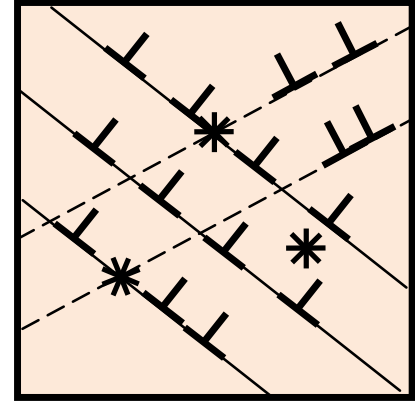

D

A

B

$\perp$ : dislocation

* : nucleation sources

Figure 8 
(a)

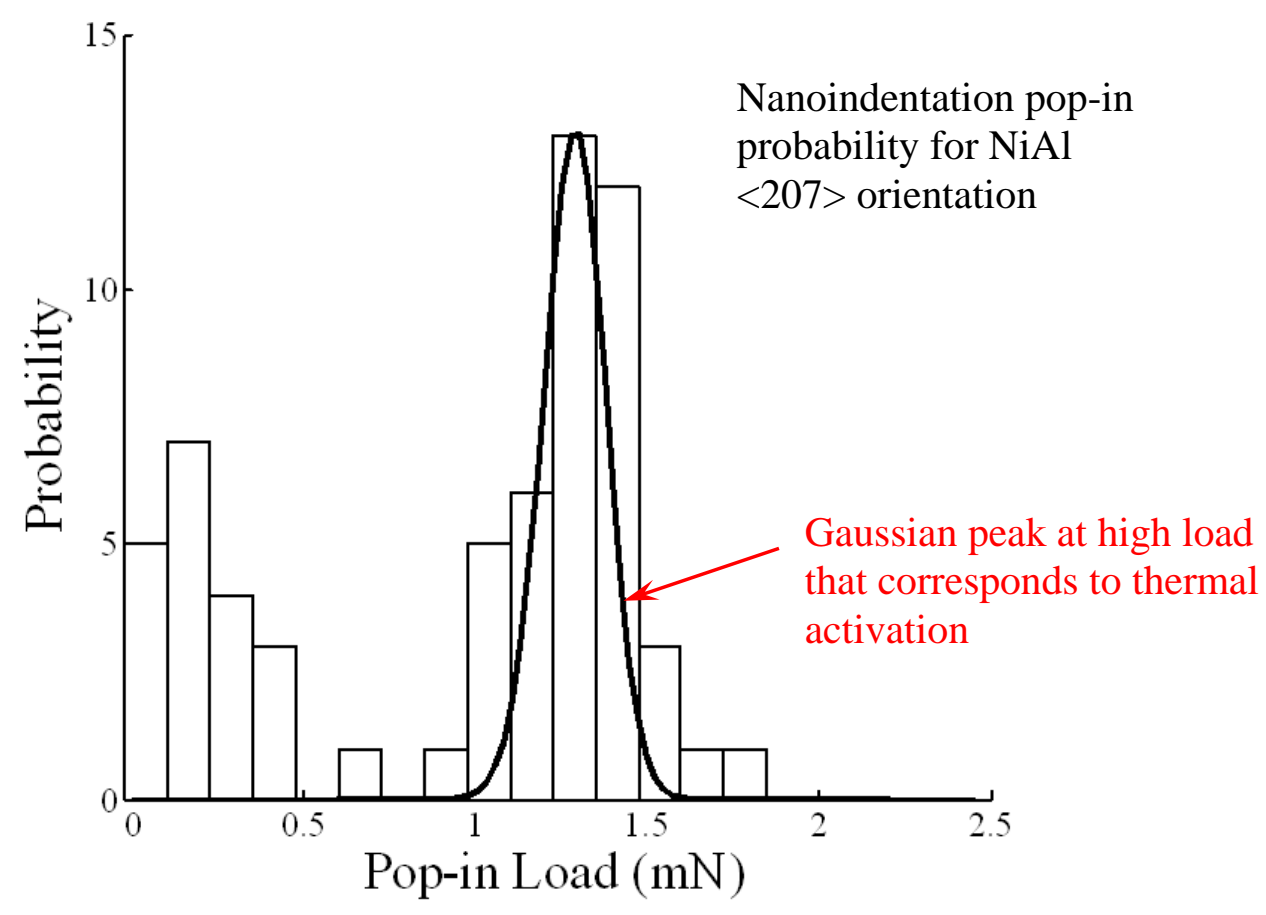

(b)

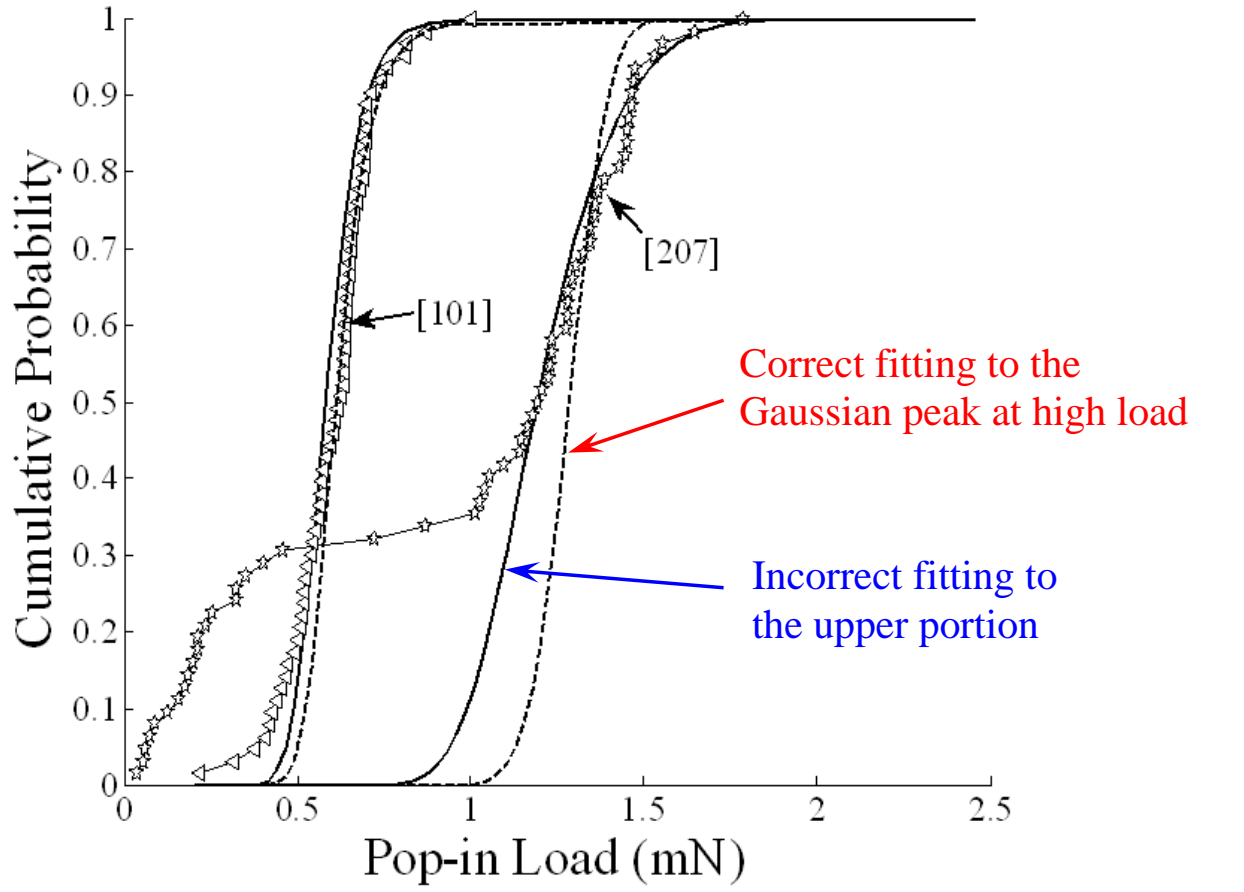

Figure 9 


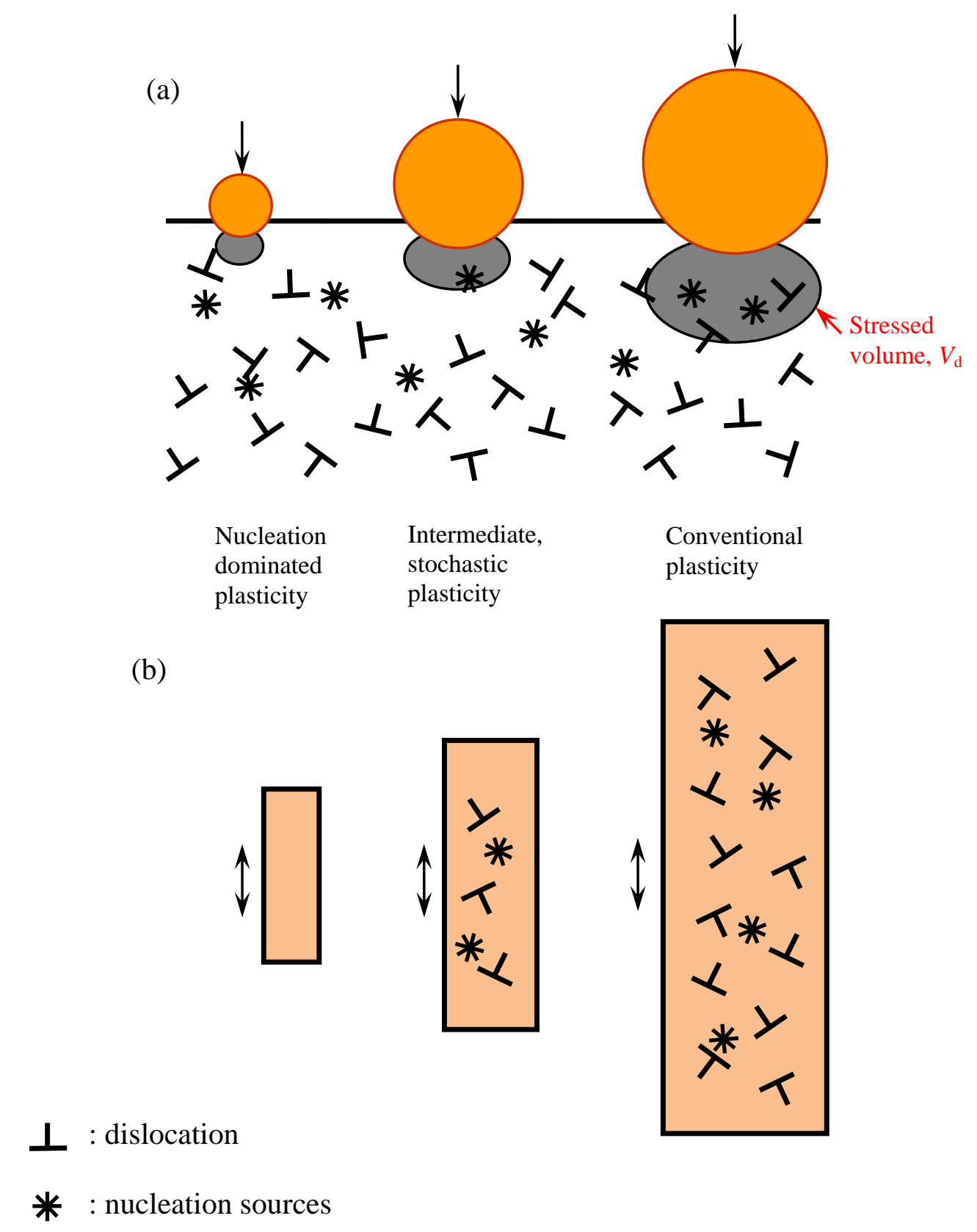

Figure 10 
(a)
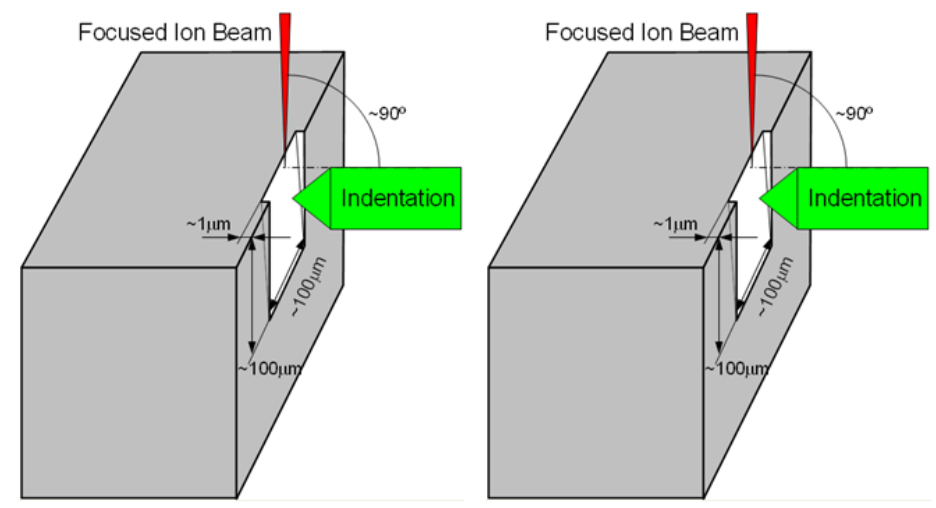

(b)

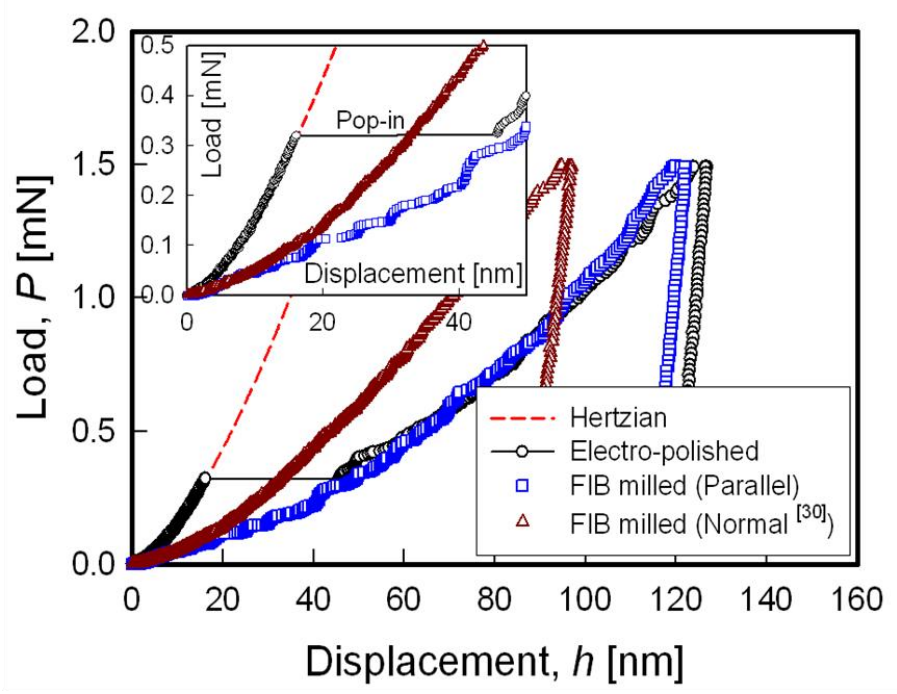

(c)

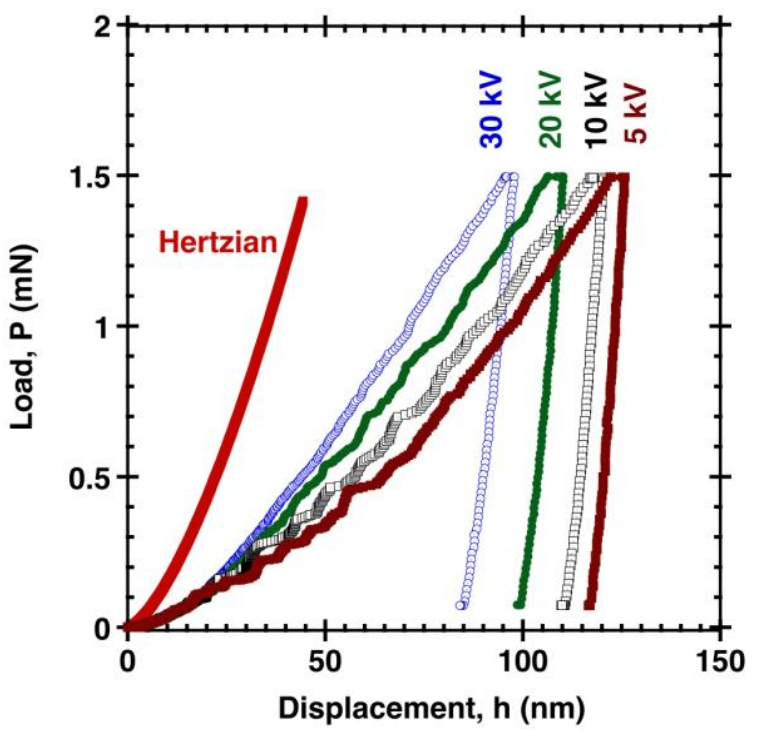

Figure 11 
(a)
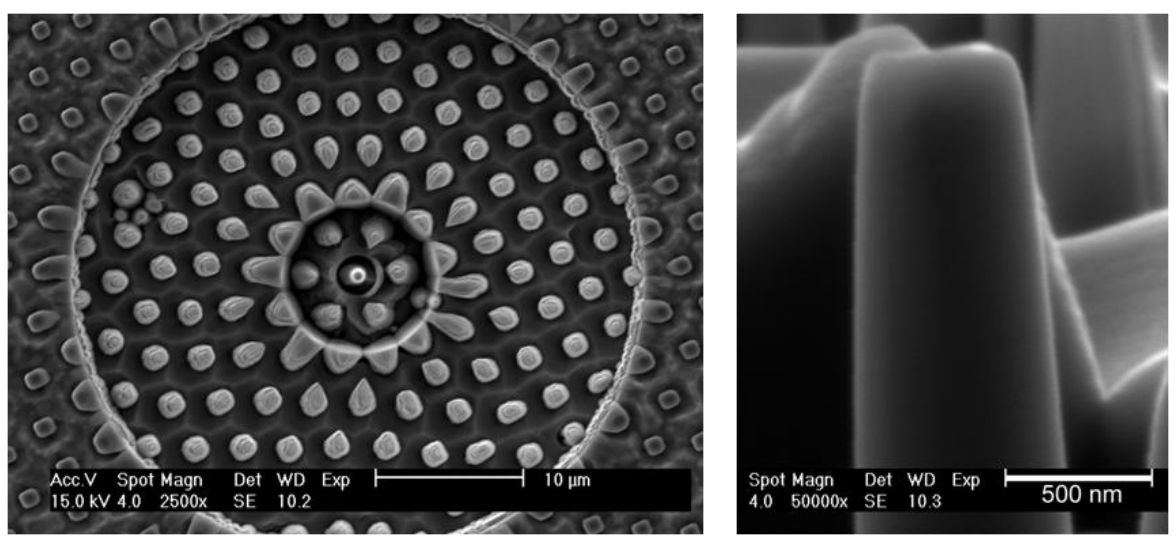

(b)

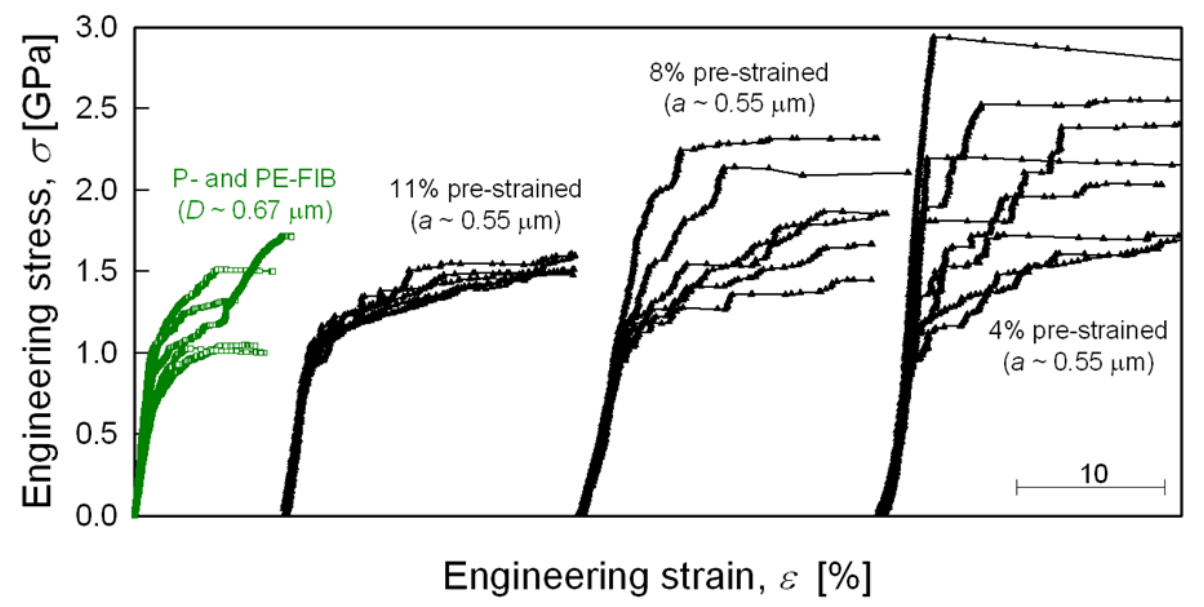

(c)

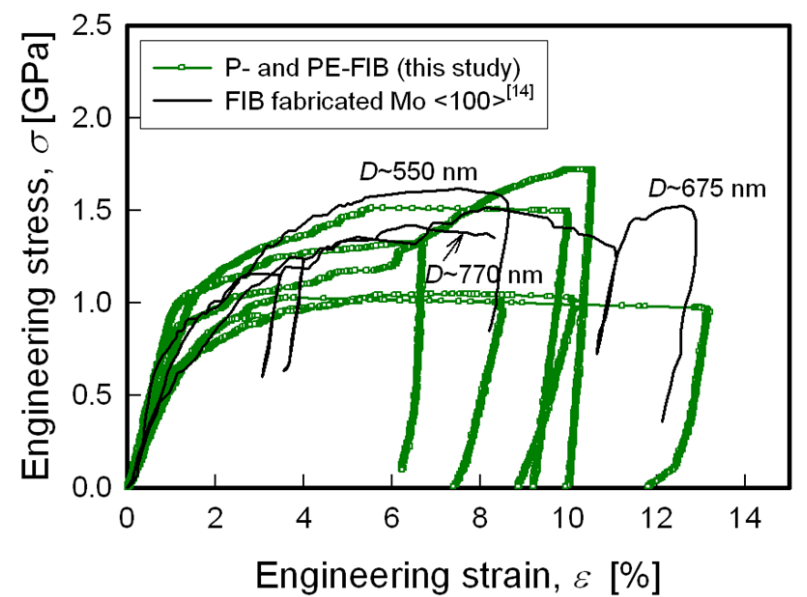

(d)
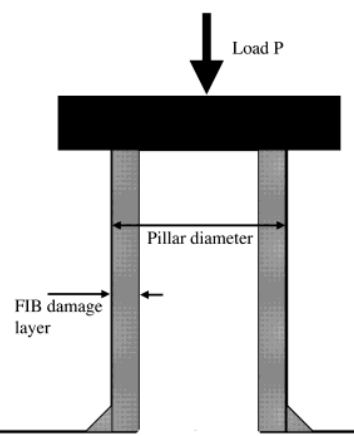

Figure 12 


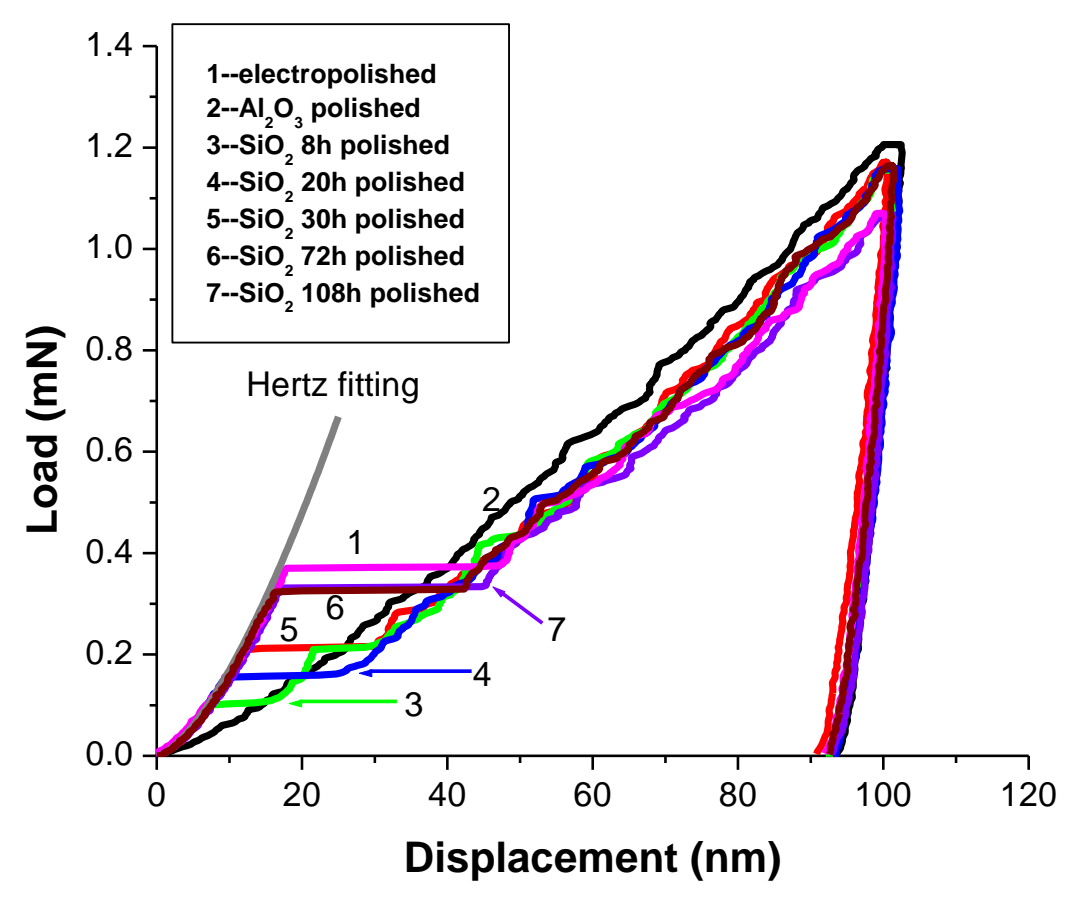

(a)

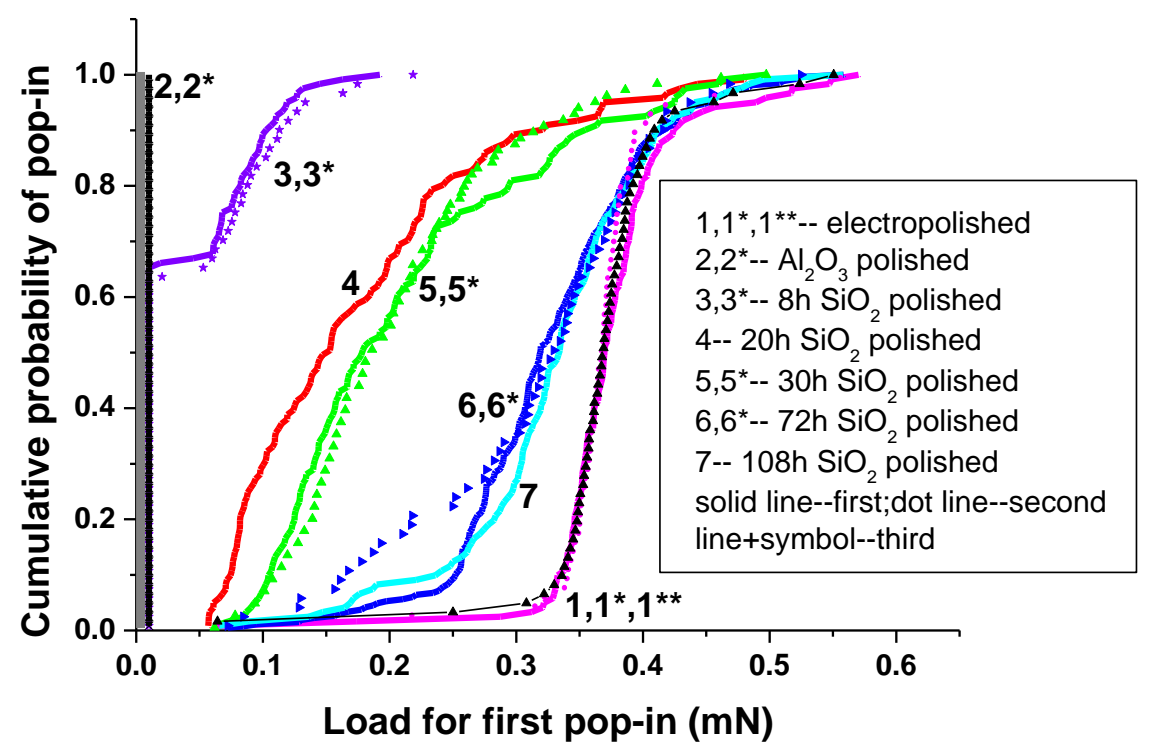

(b)

Figure 13 

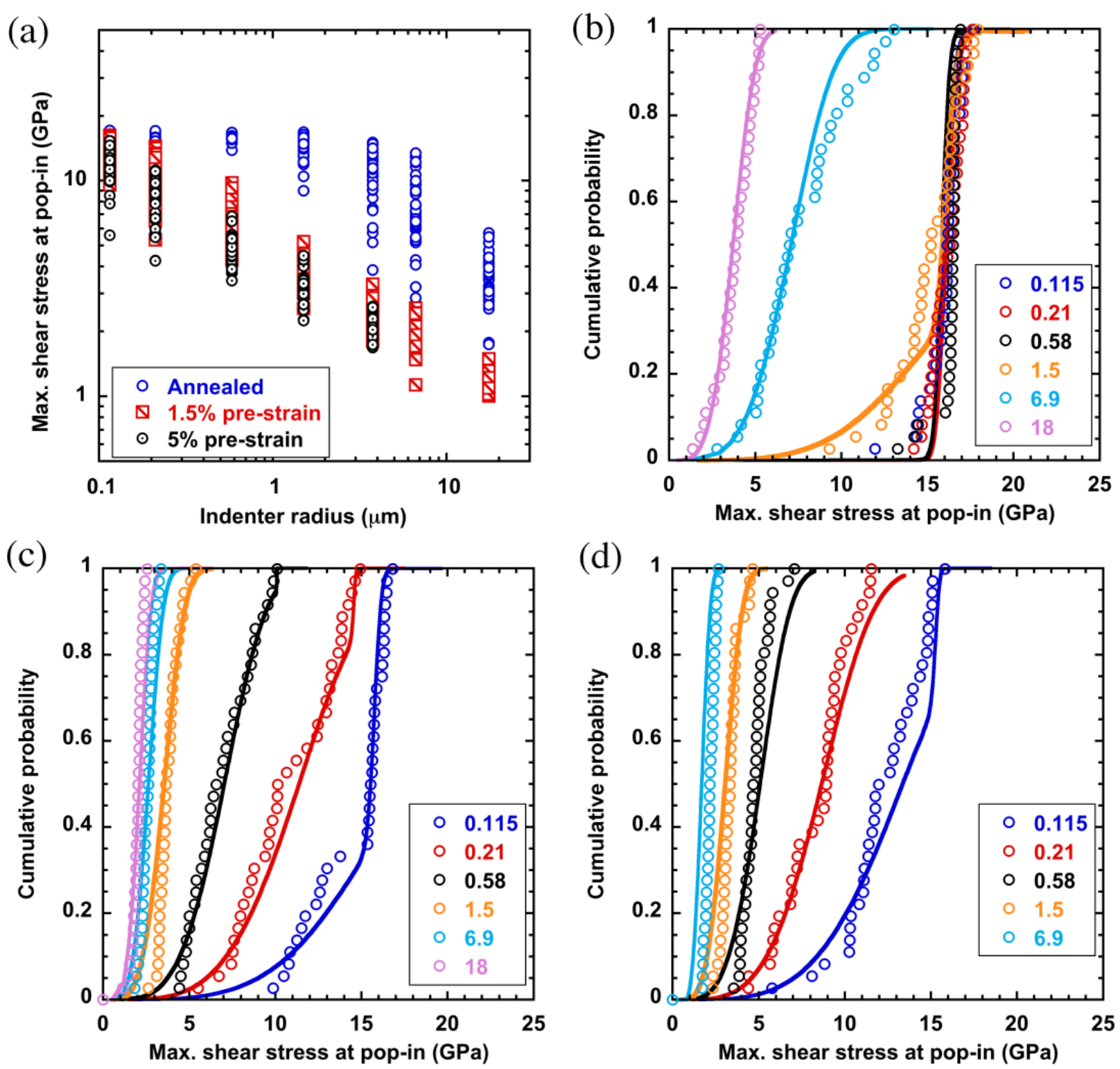

Figure 14 


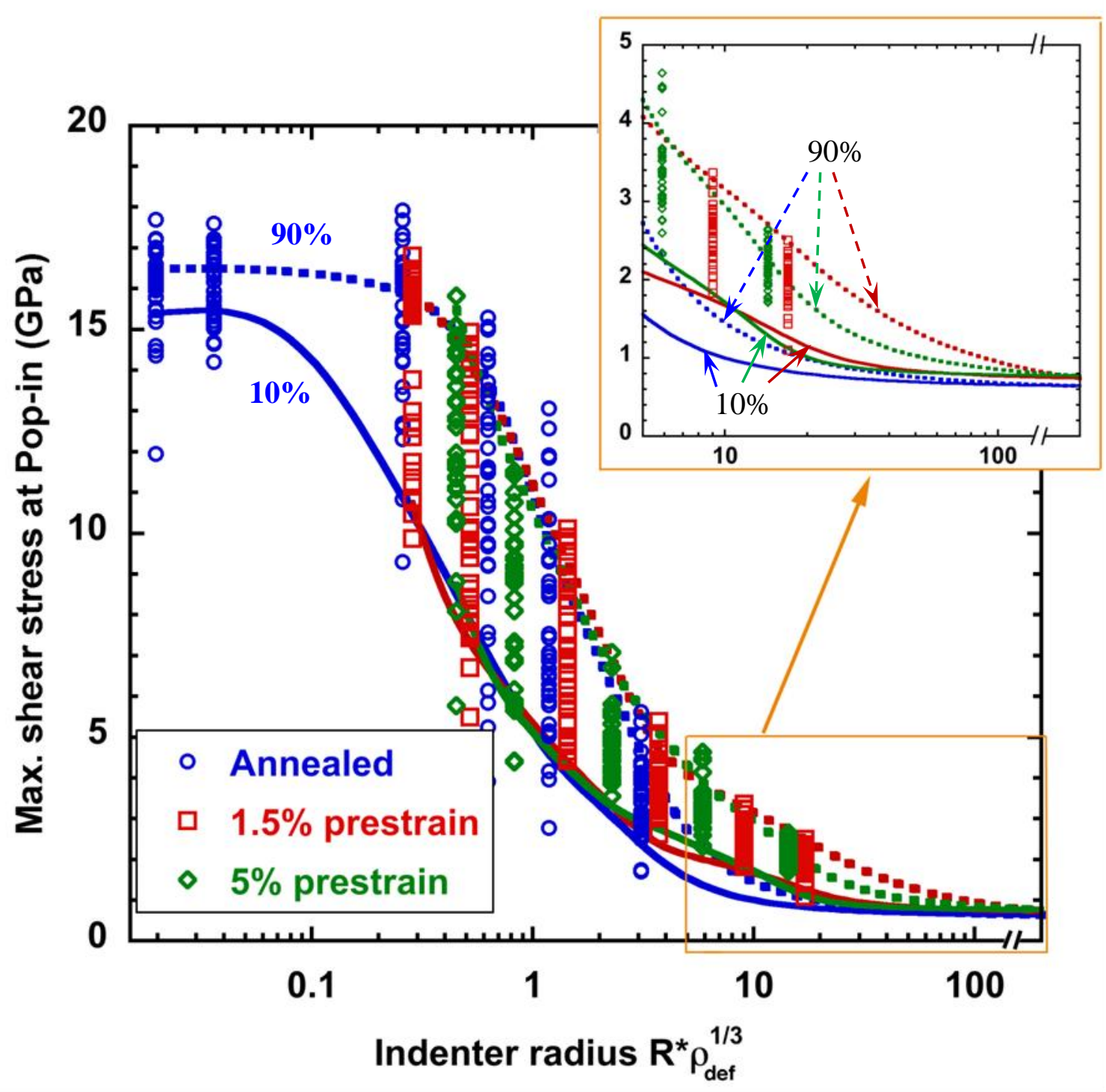

Figure 15 
(a)

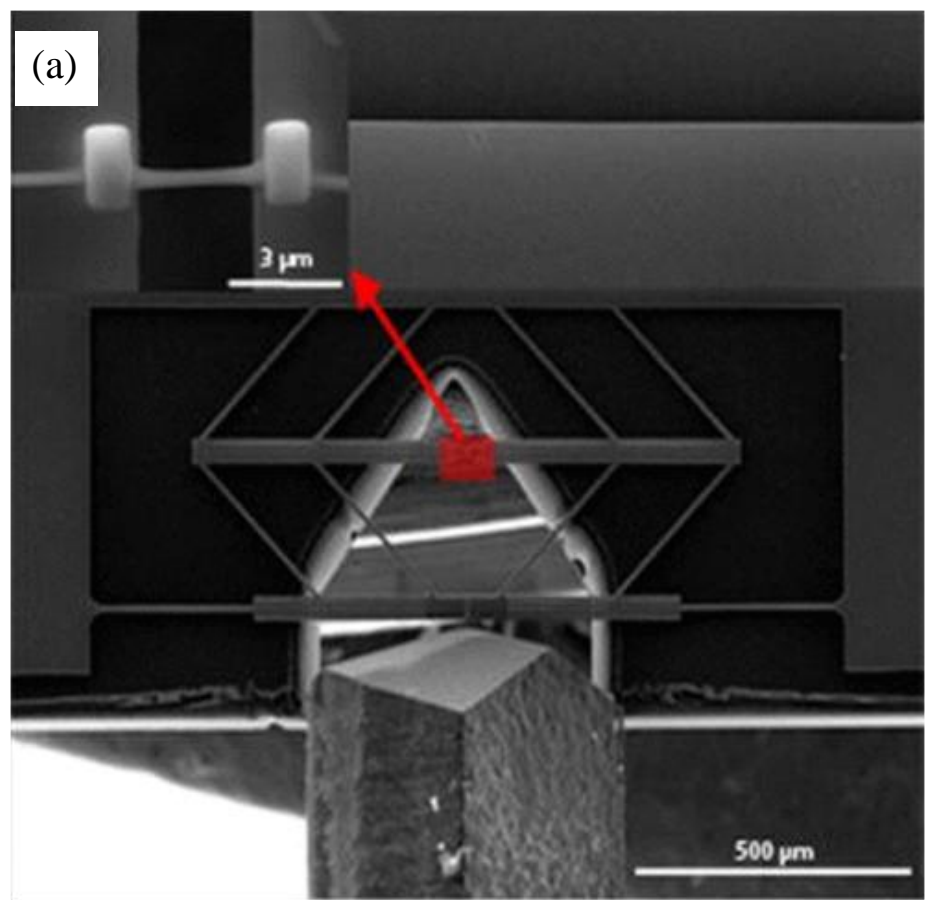

(b)

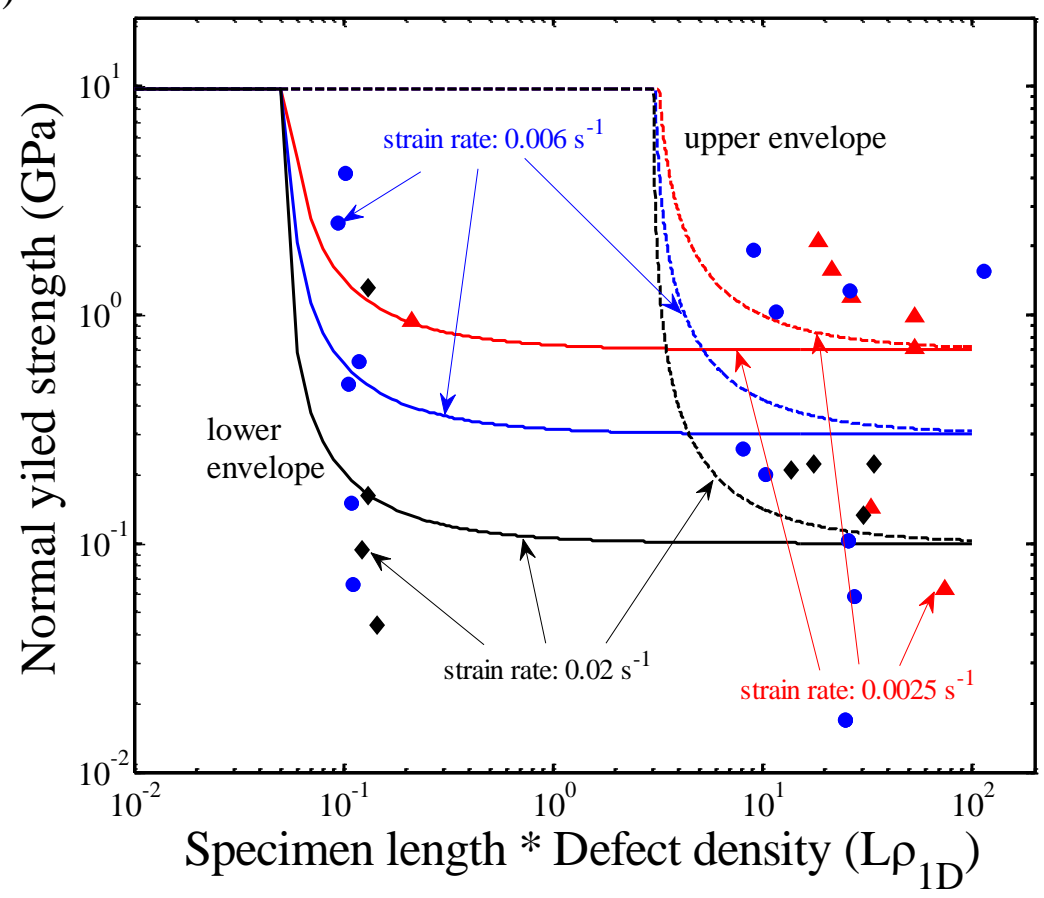

Figure 16 
(a)

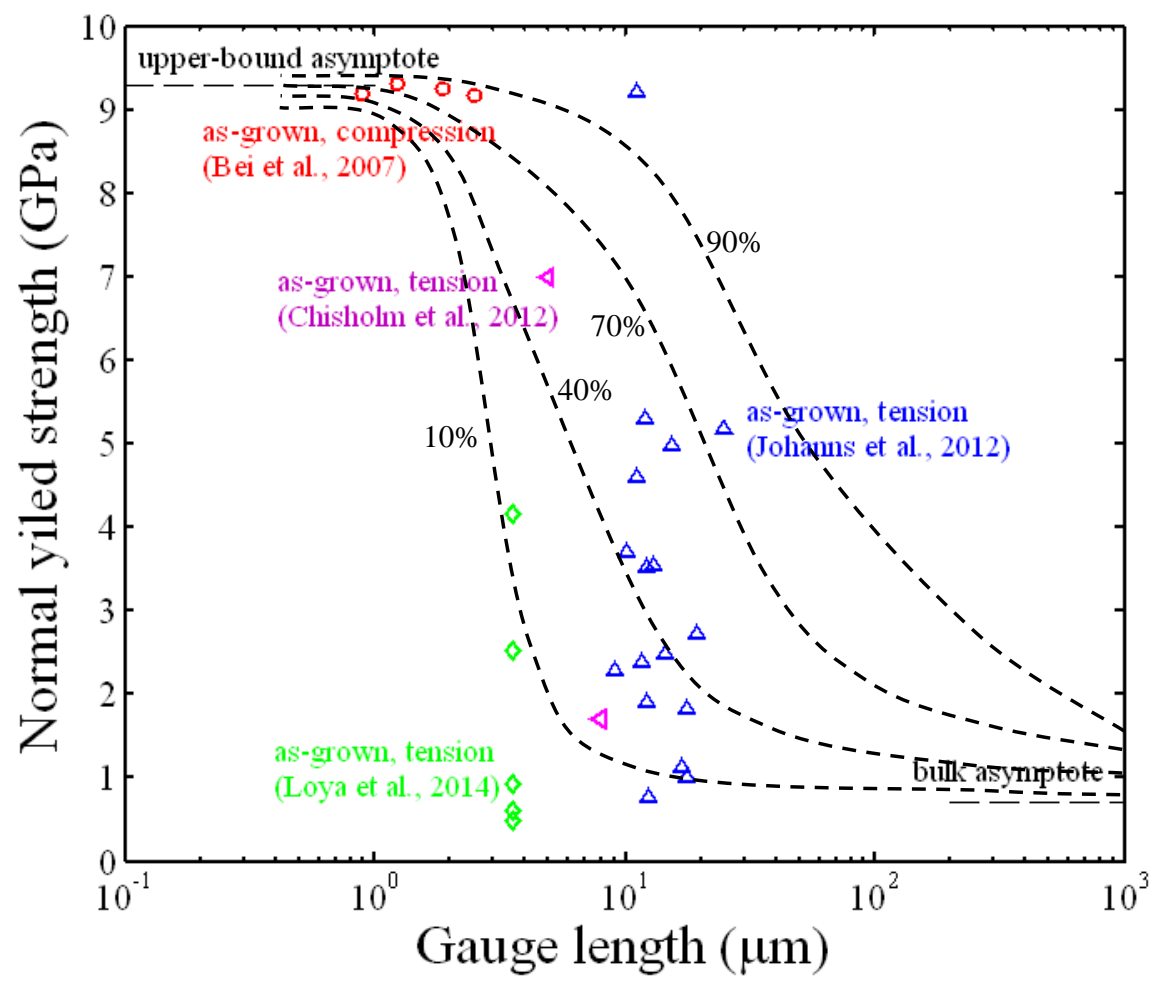

(b)

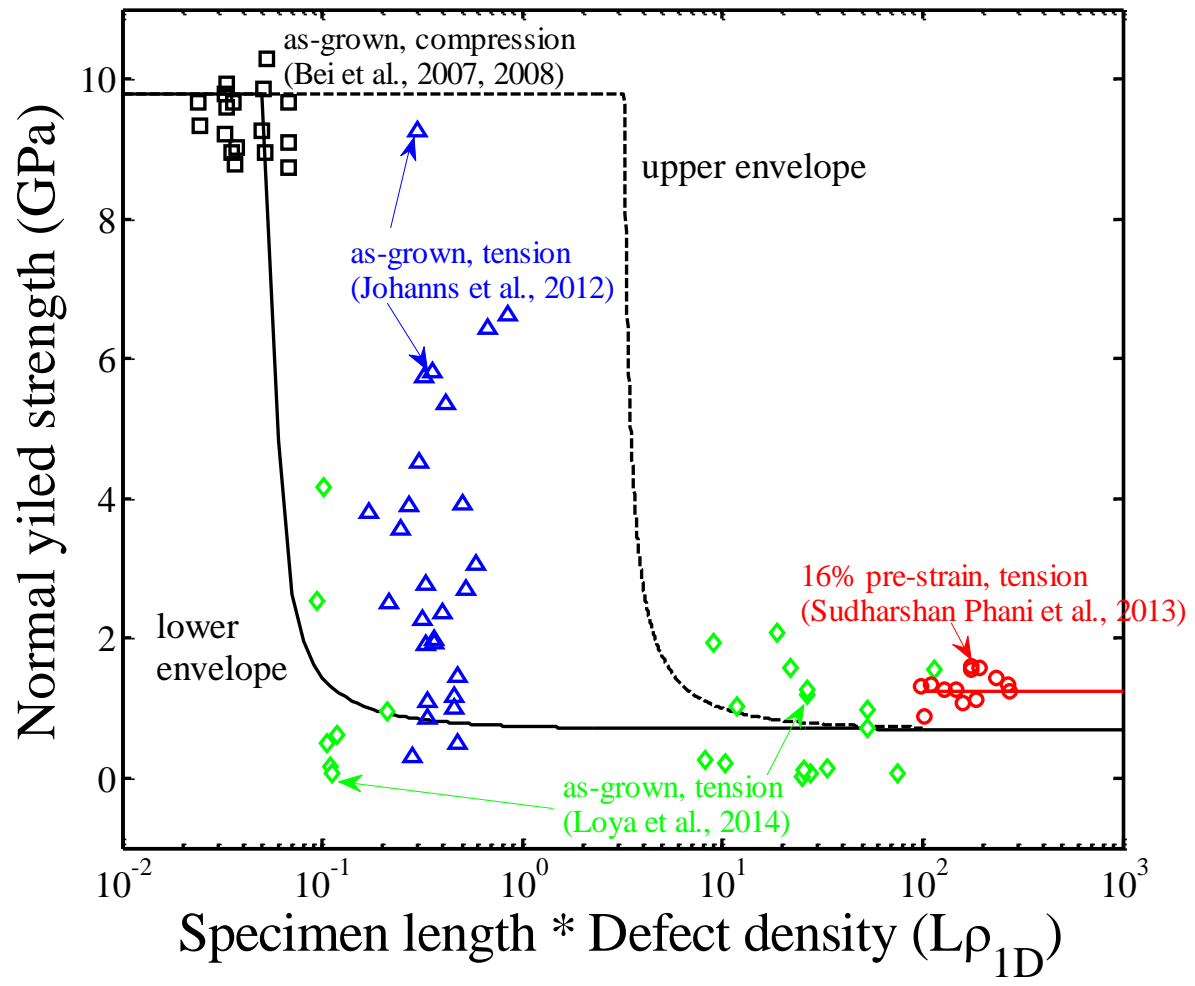

Figure 17 


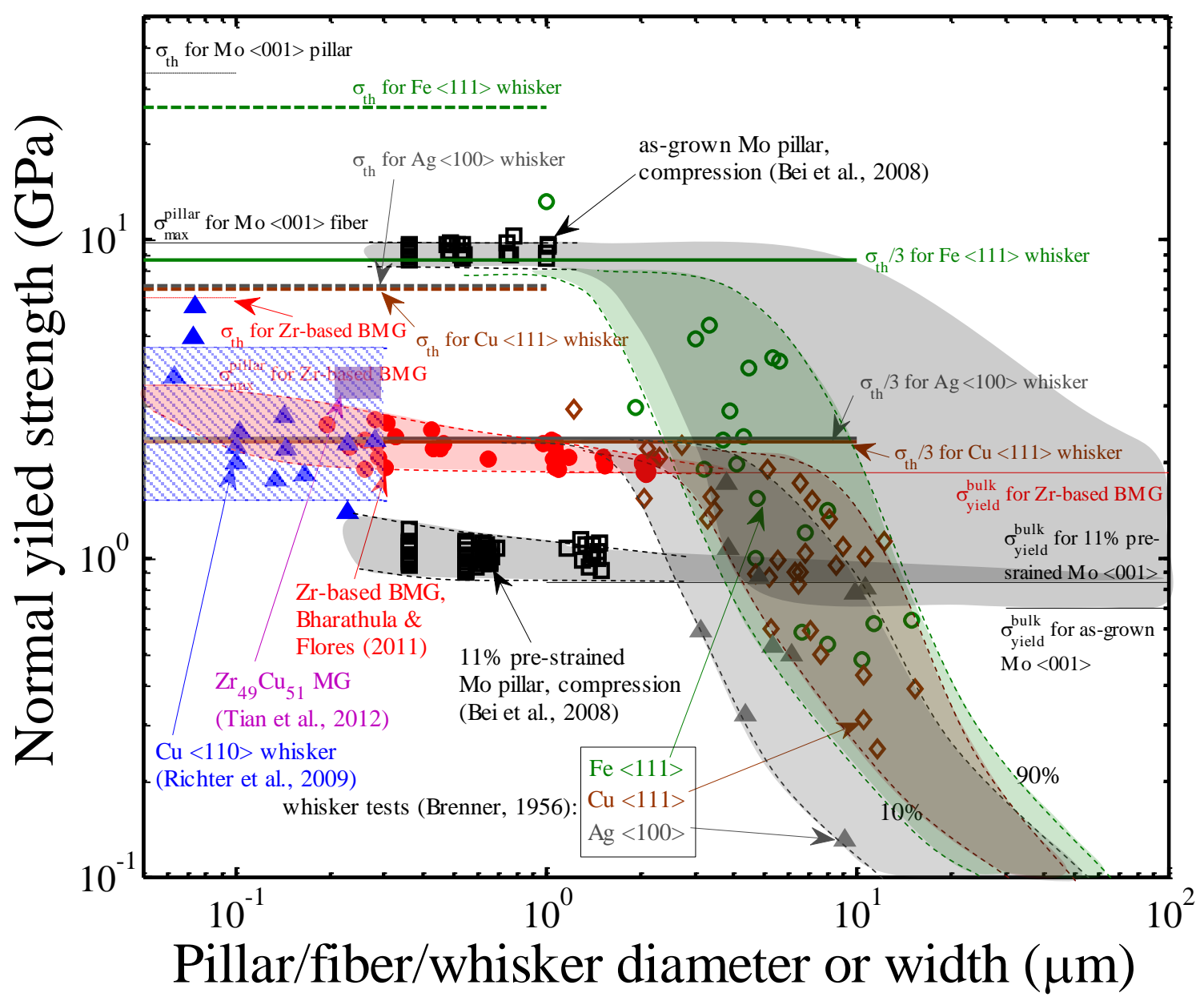

Figure 18 

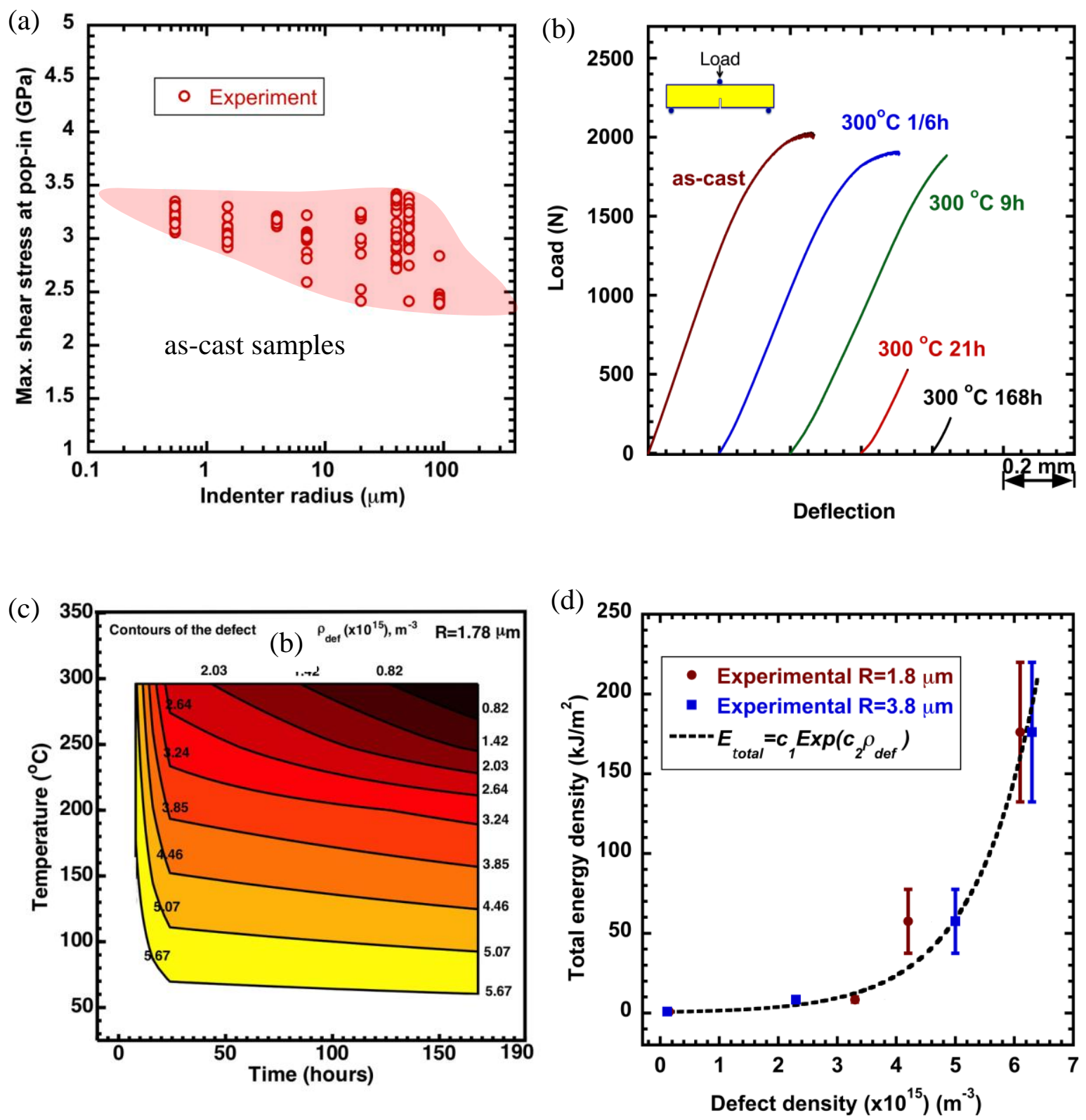

Figure 19 

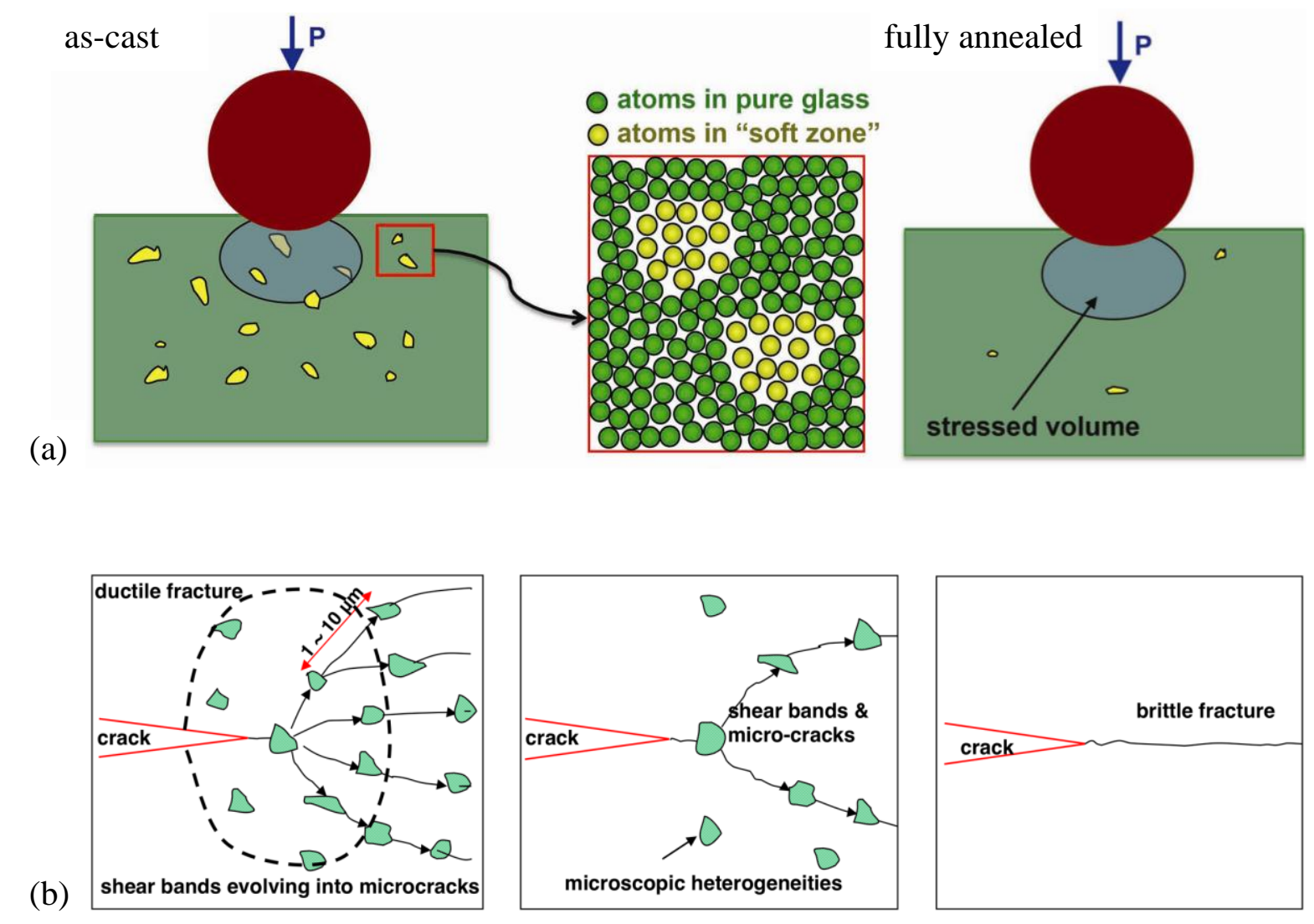

Figure 20 


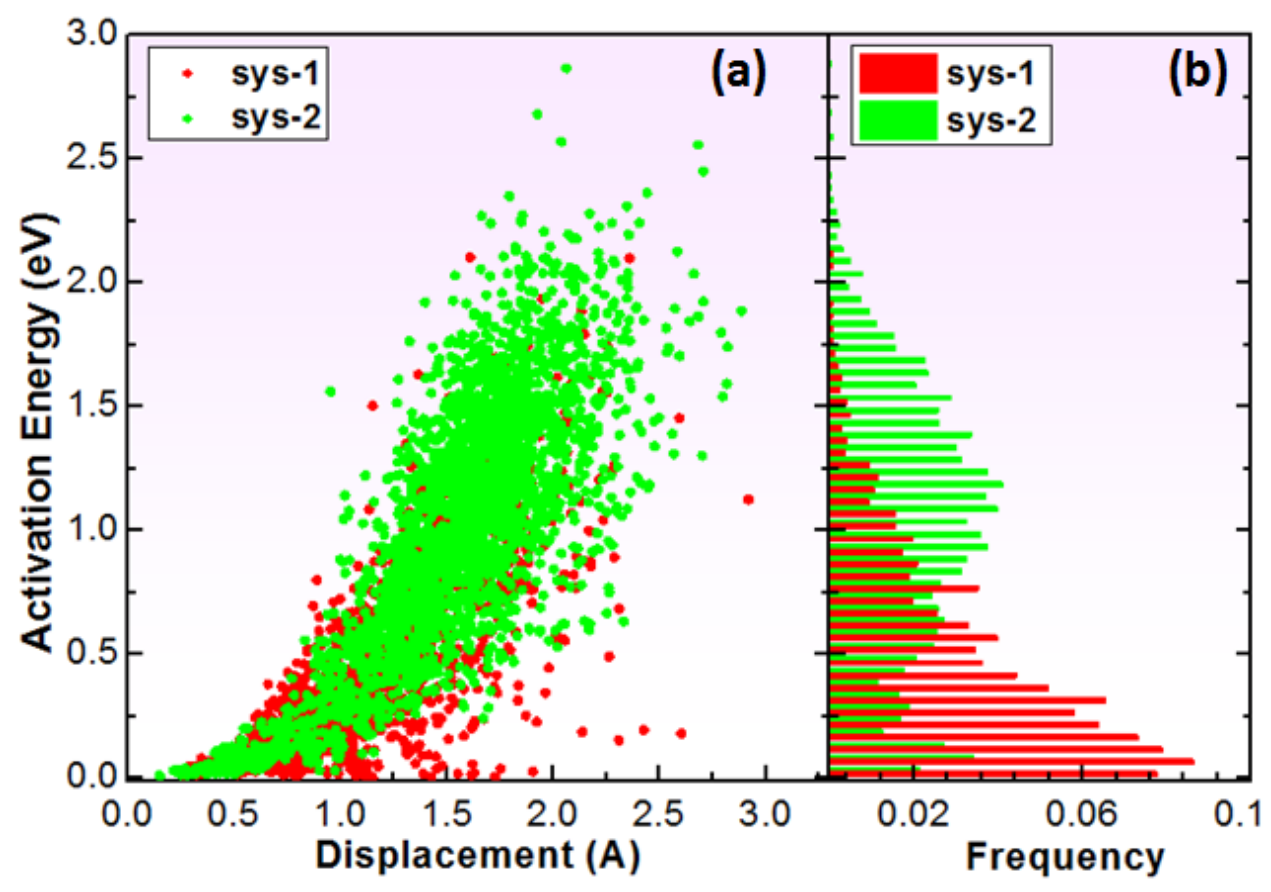

Figure 21 
(a)

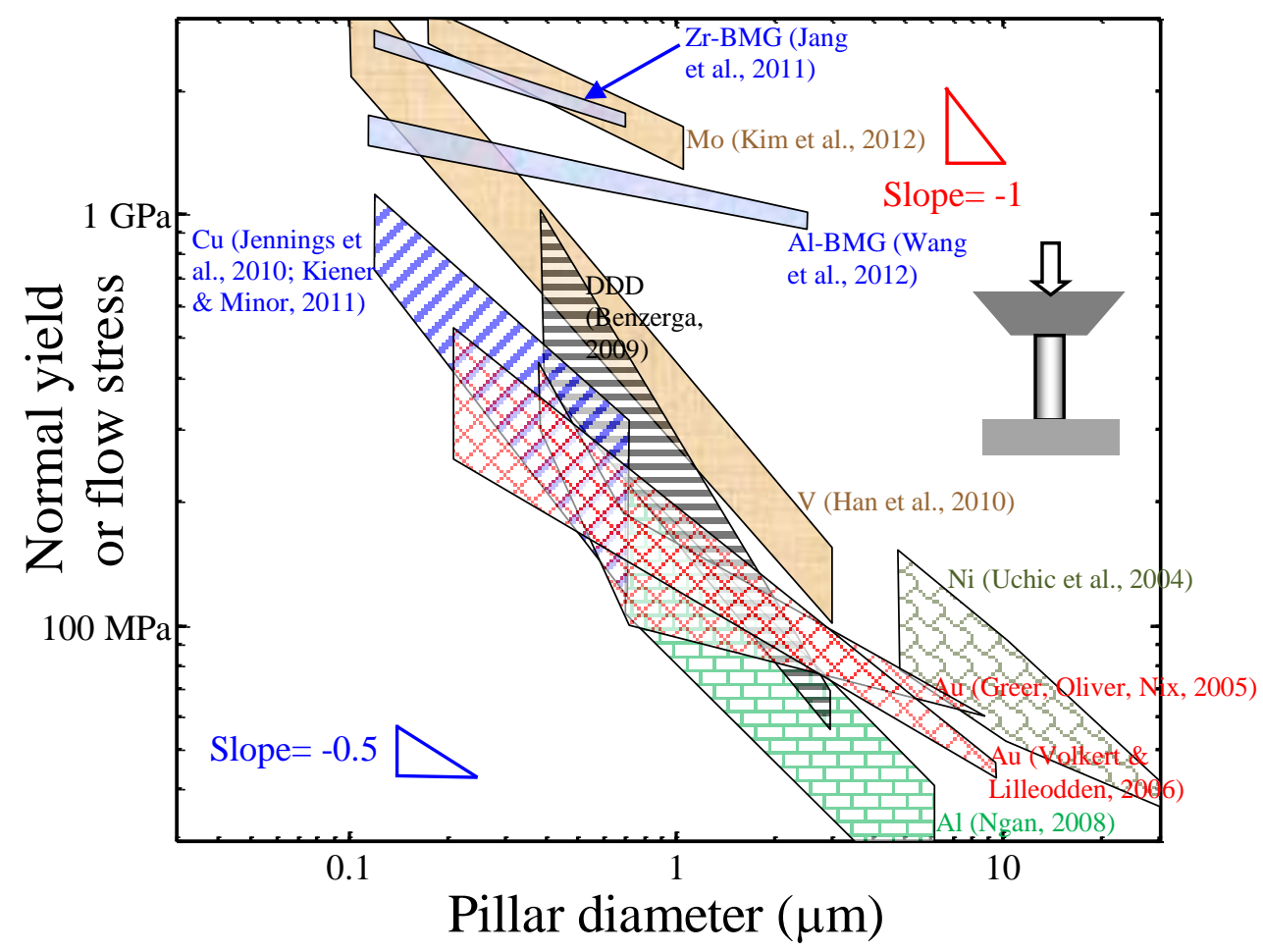

(b)

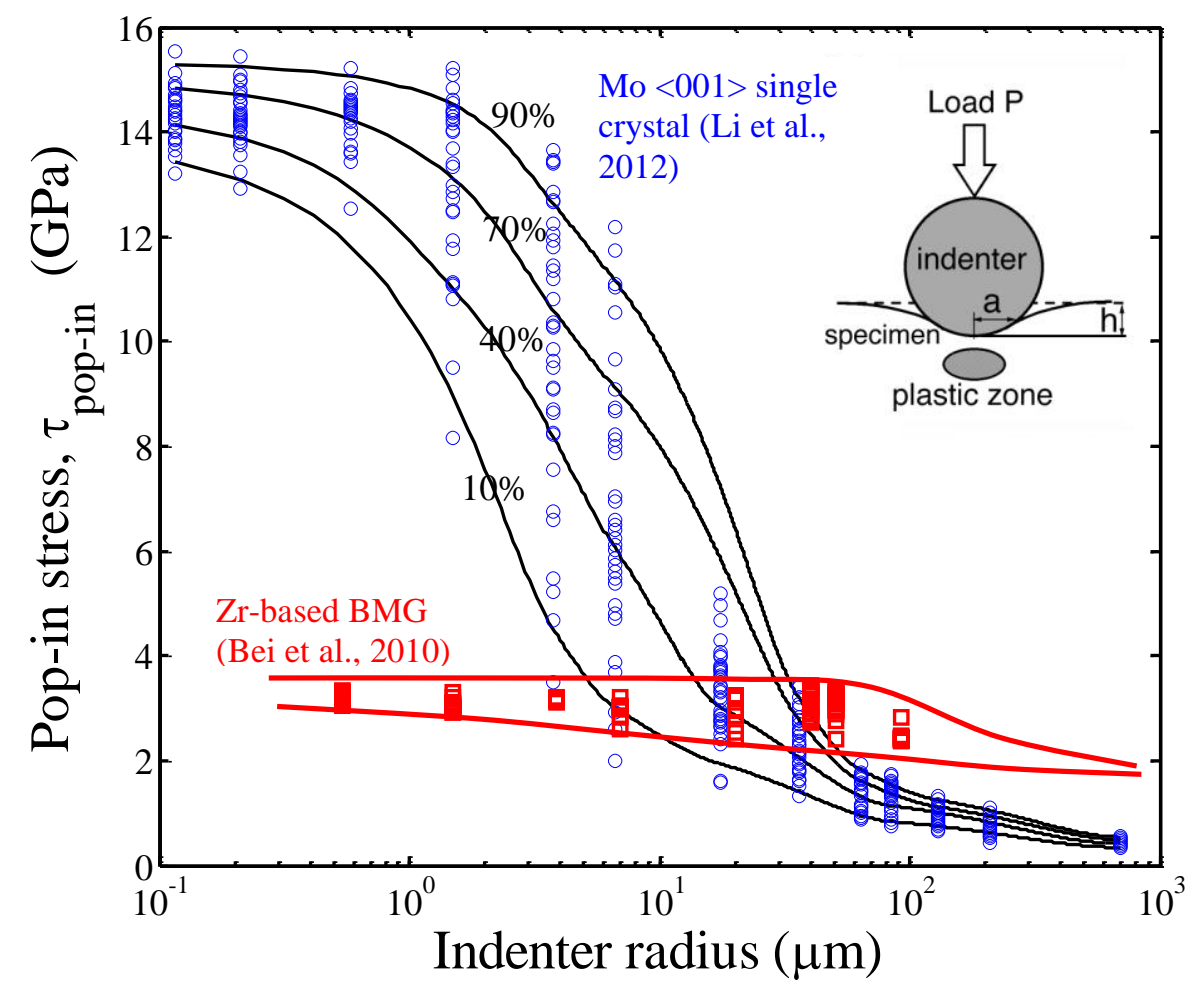

Figure 1 
(c)

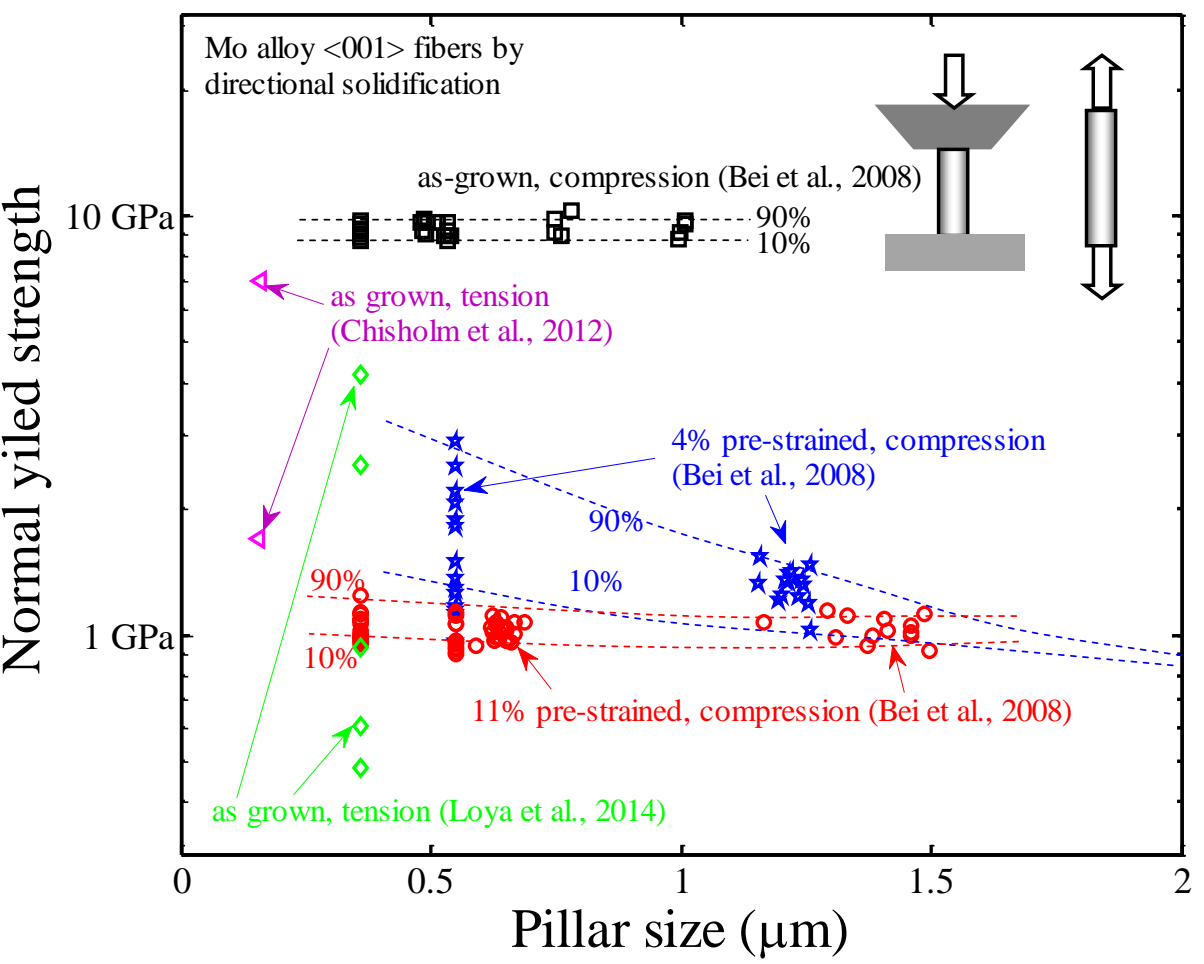

(d)

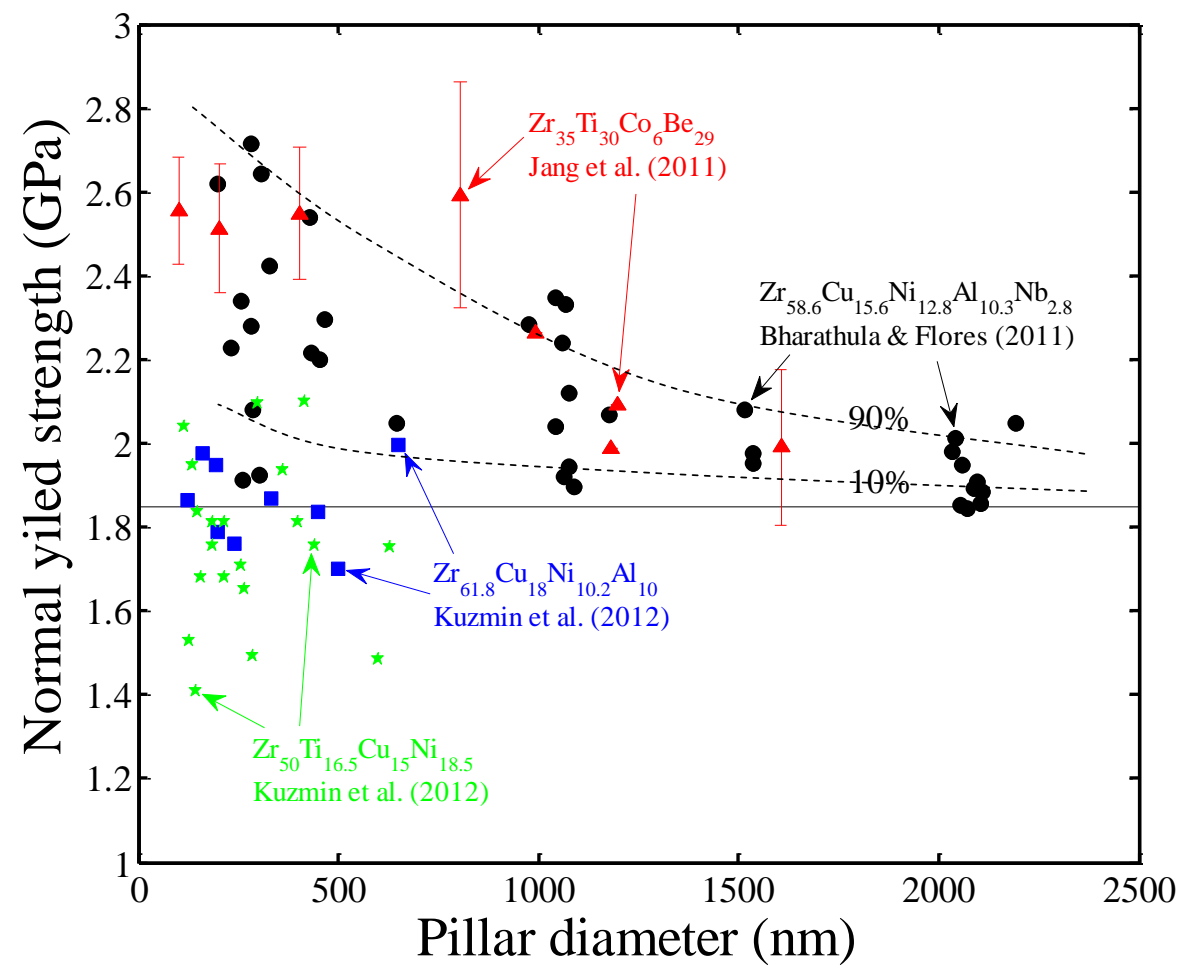

Figure 1 (cont'd) 
(a)

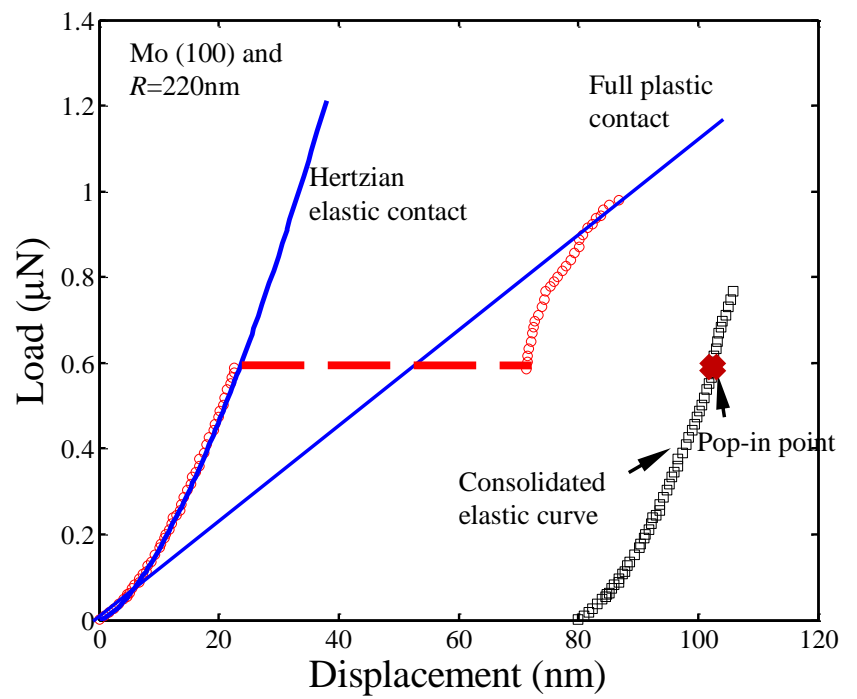

(b)

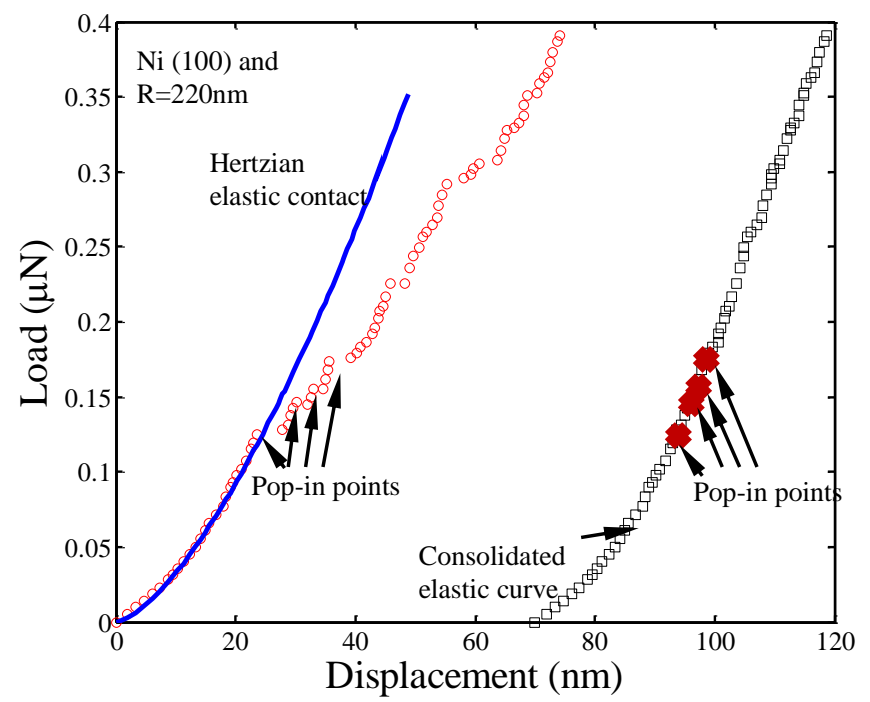

(c)

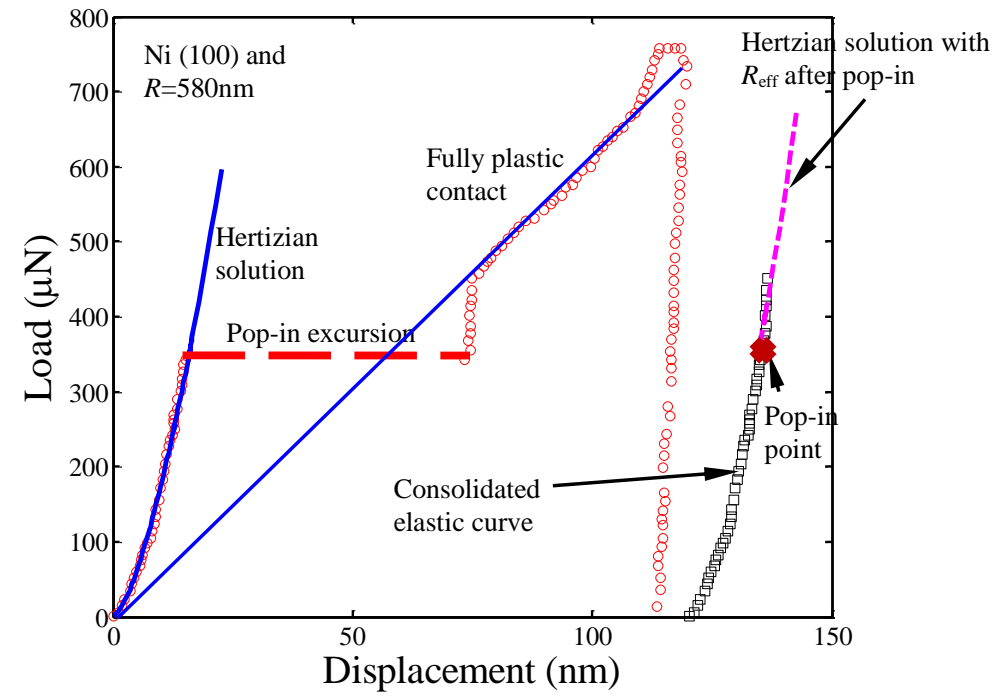

Figure 2 
(a)
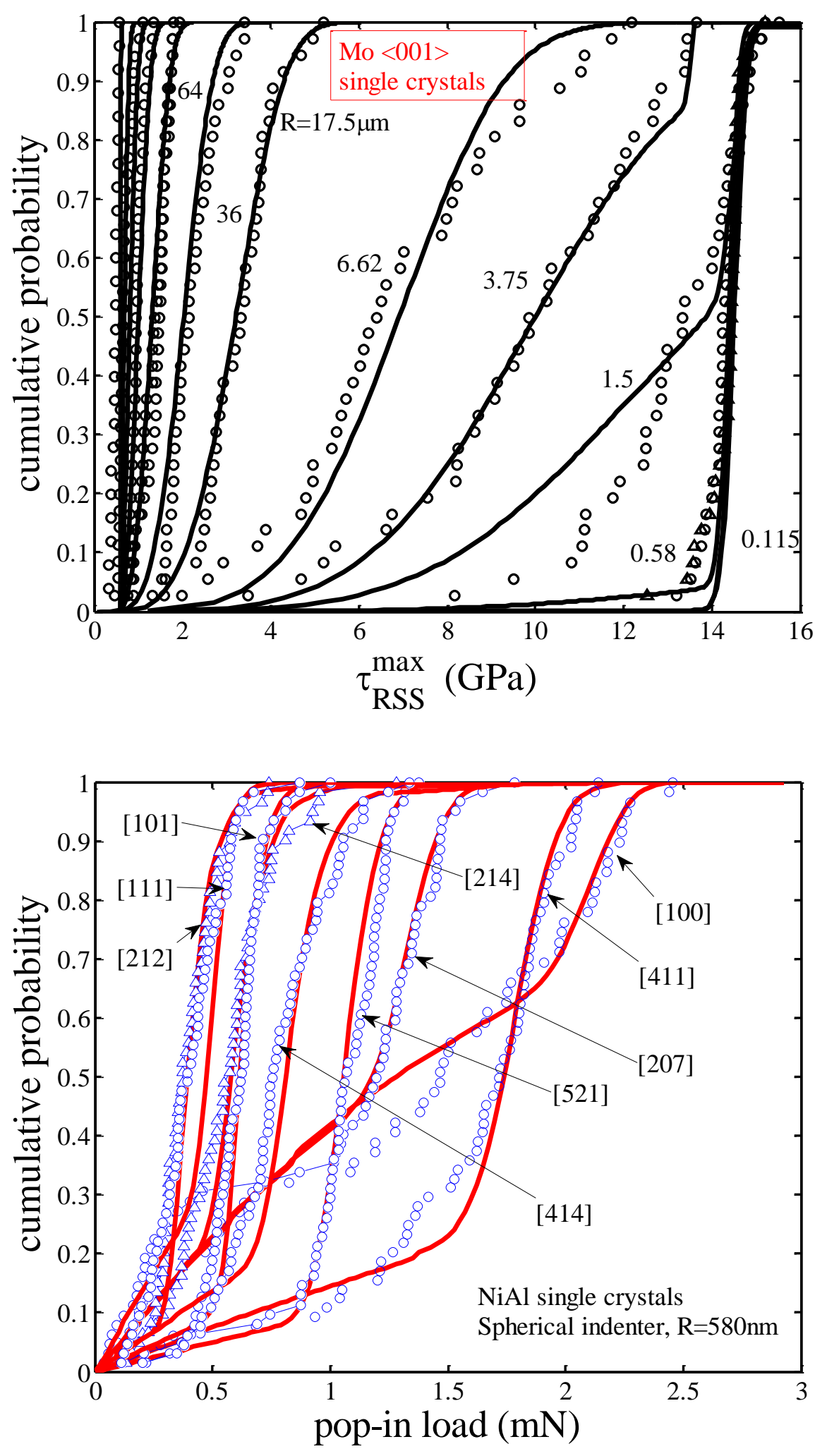

(b)

Figure 3 

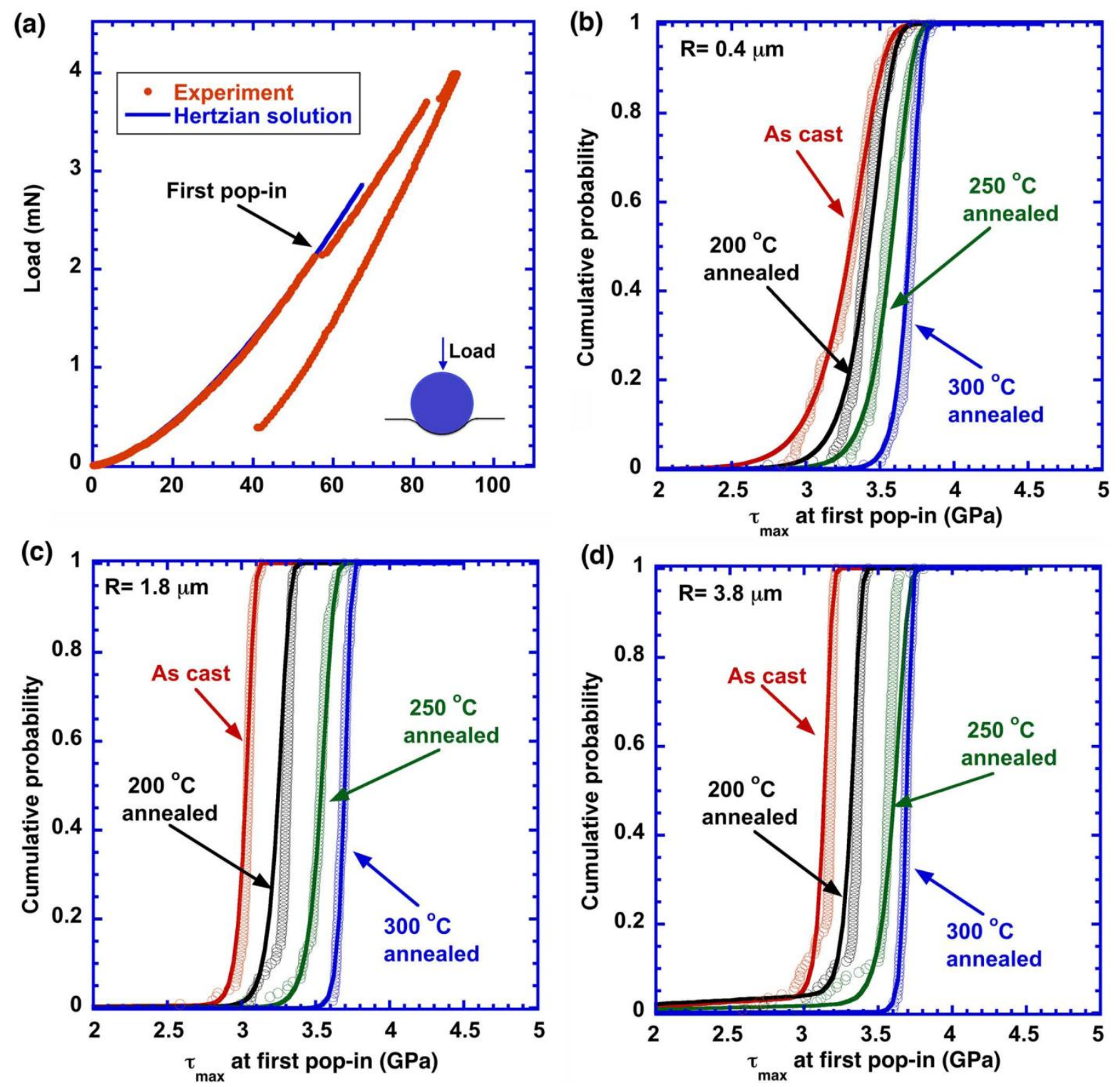

Figure 4 

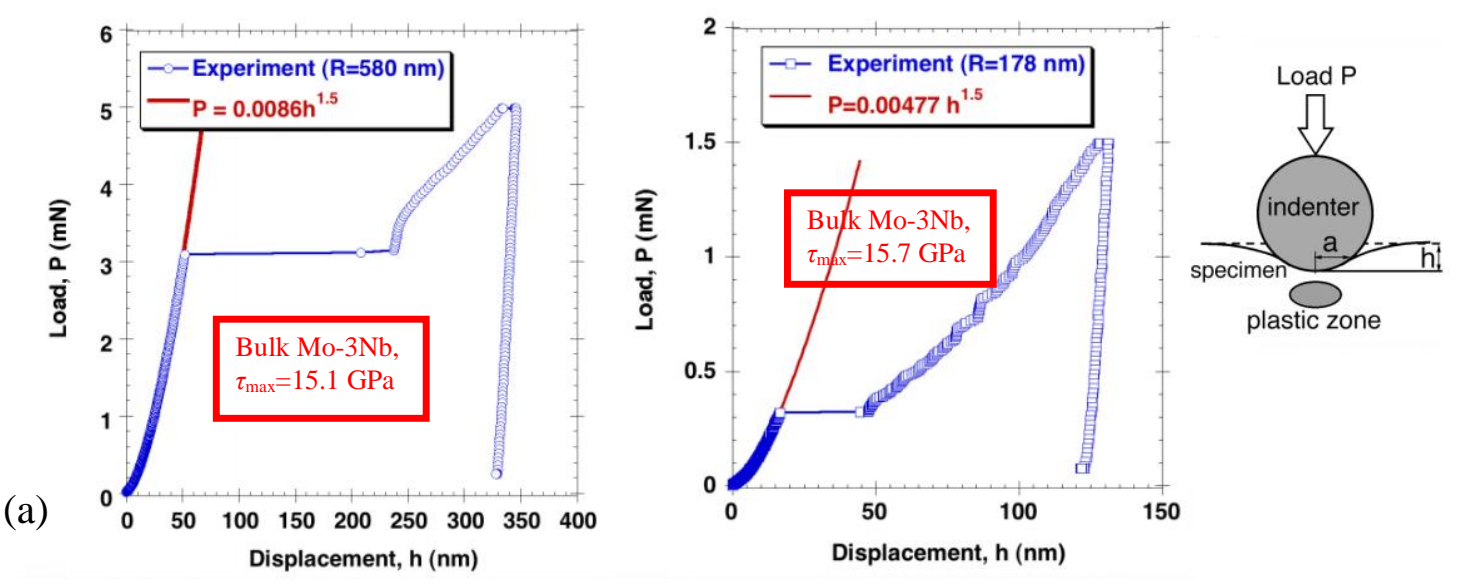

(b)
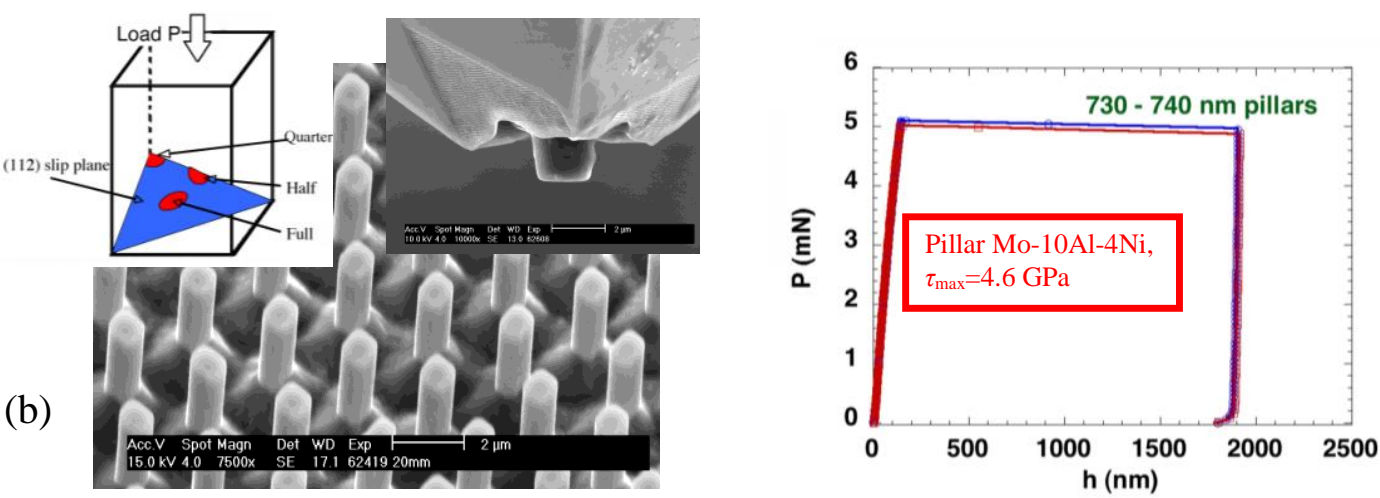

(c)
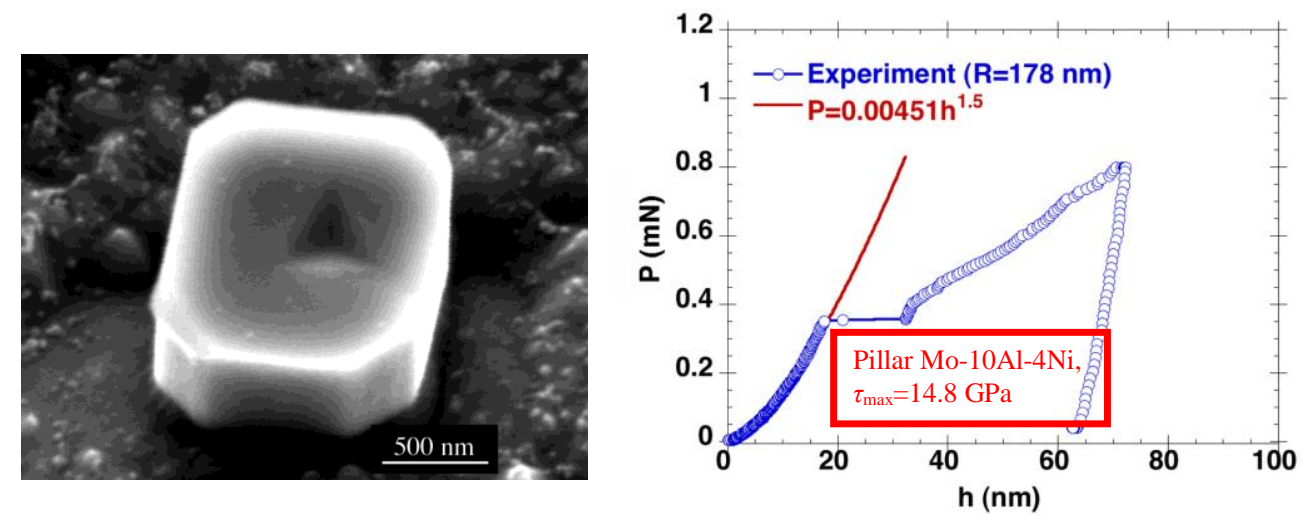

Figure 5 

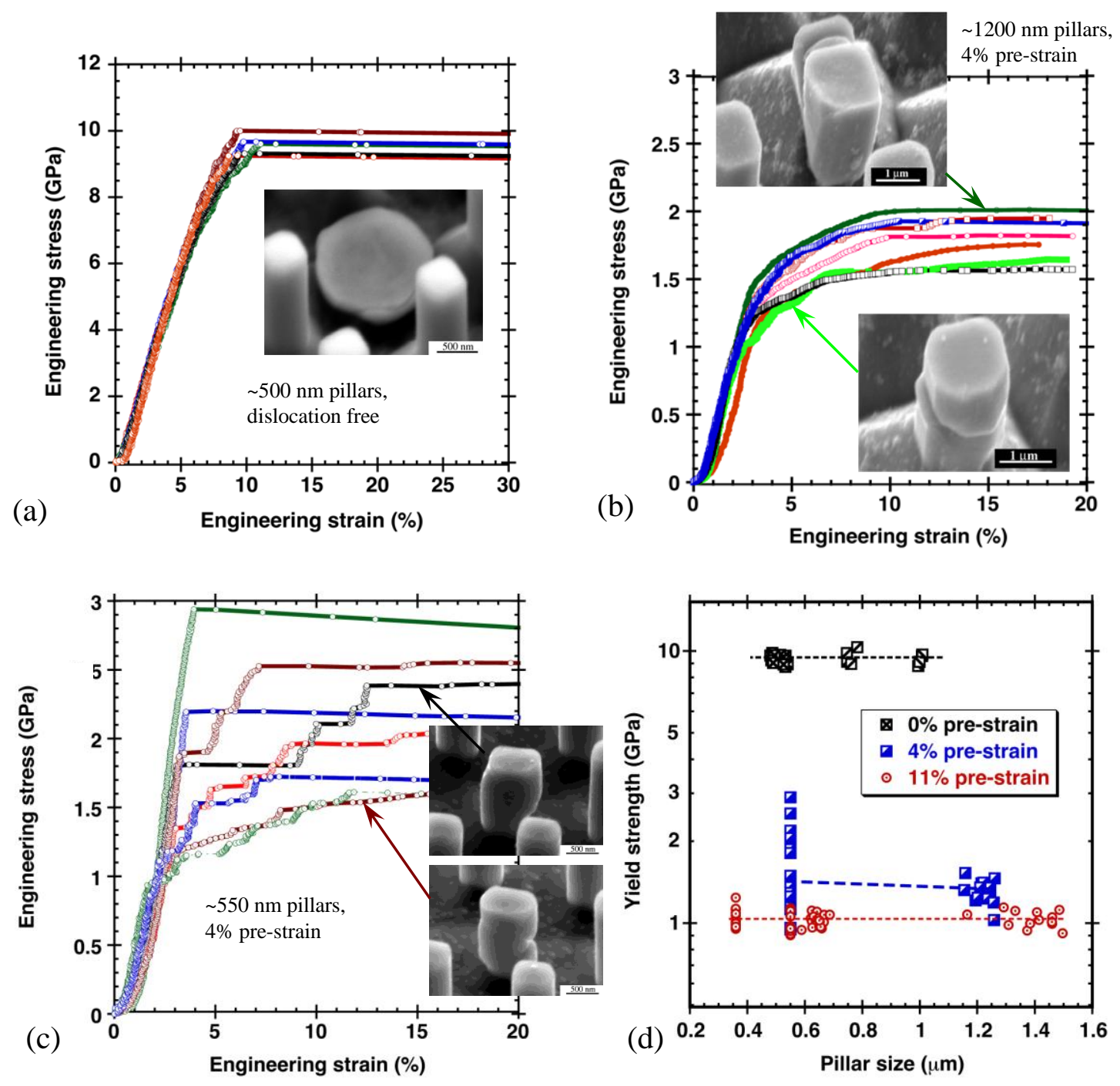

Figure 6 


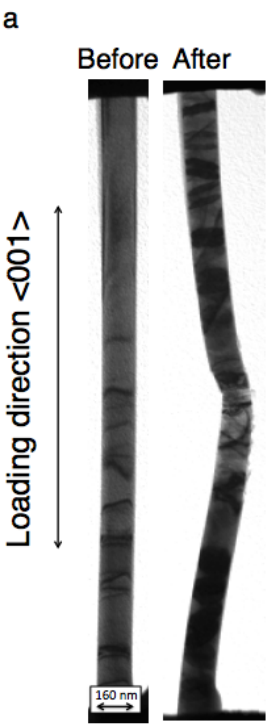

b

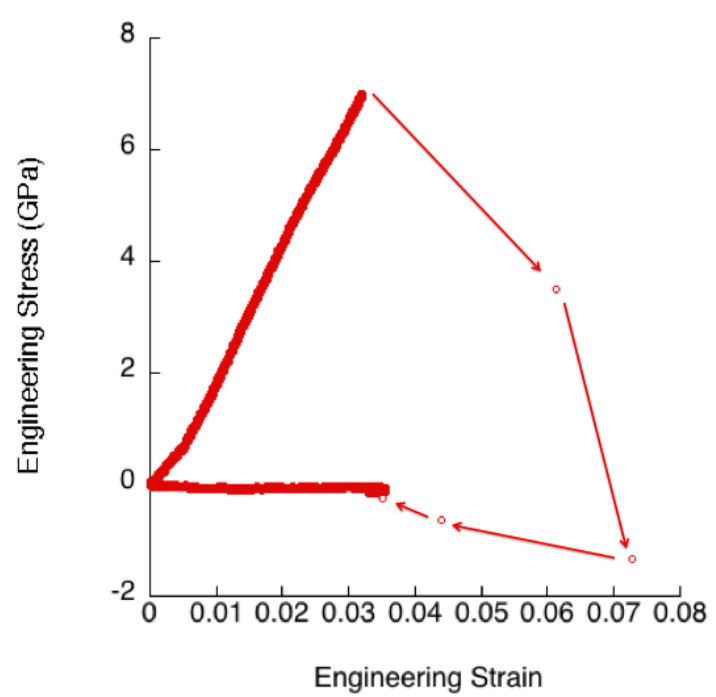

(a)

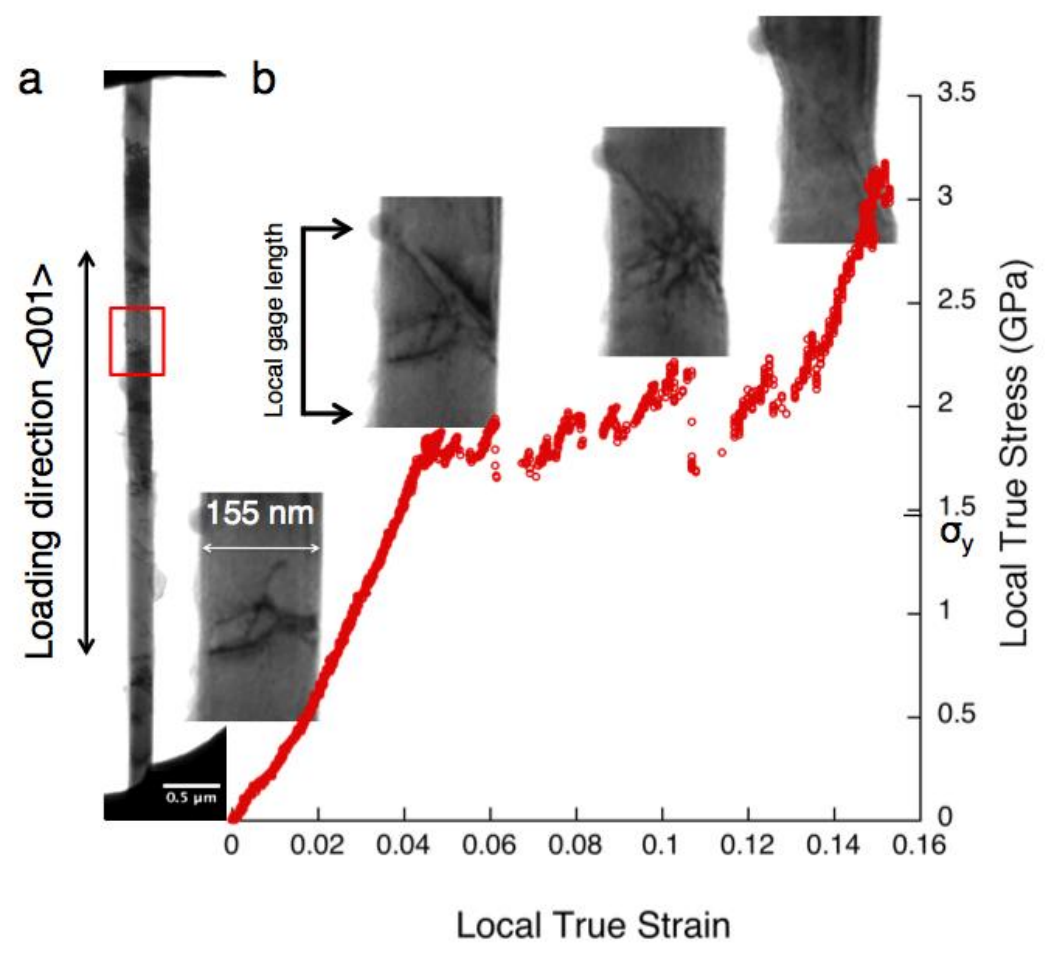

Figure 7 

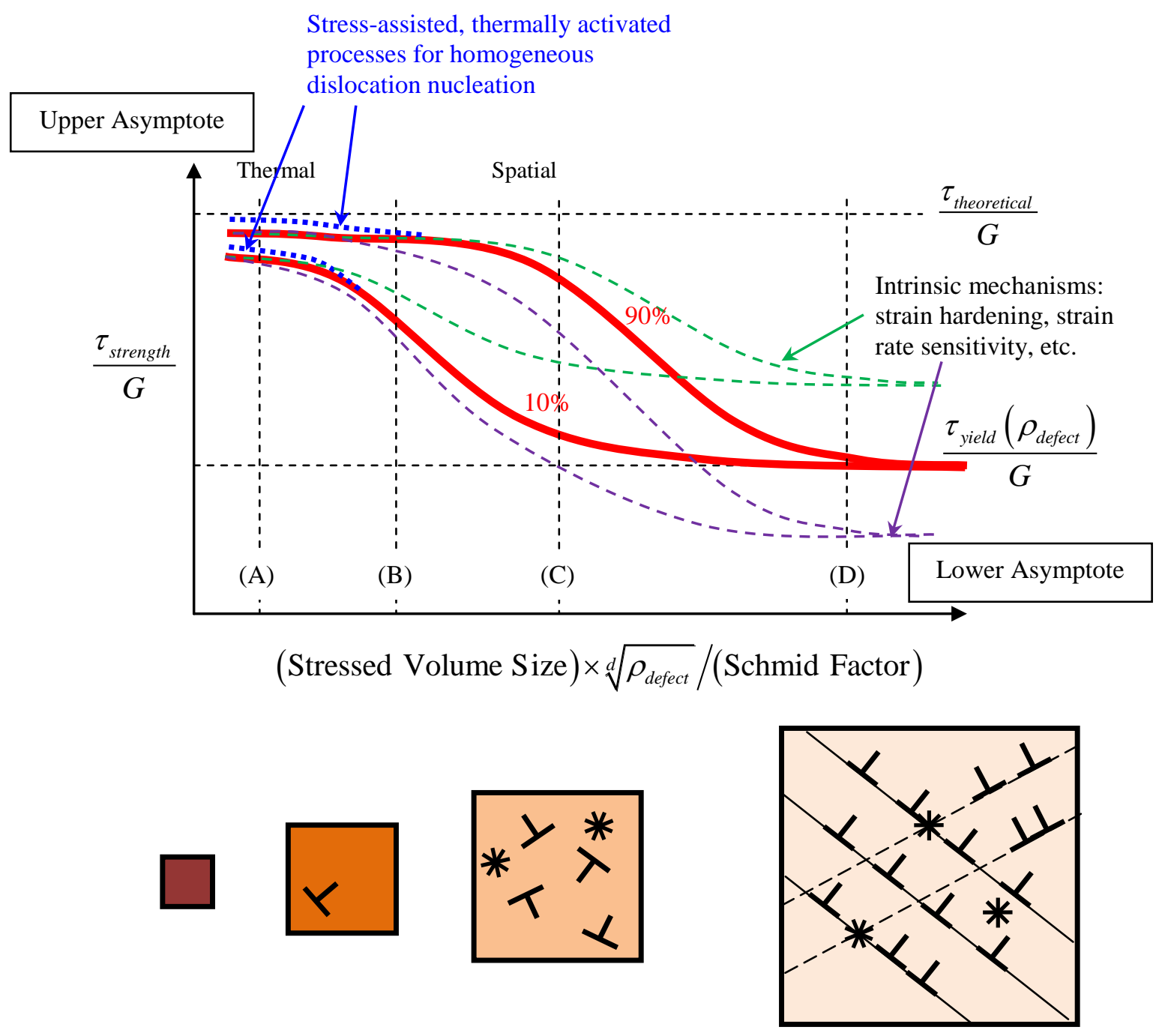

A

B

C

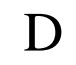

$\perp$ : dislocation

* : nucleation sources

Figure 8 
(a)

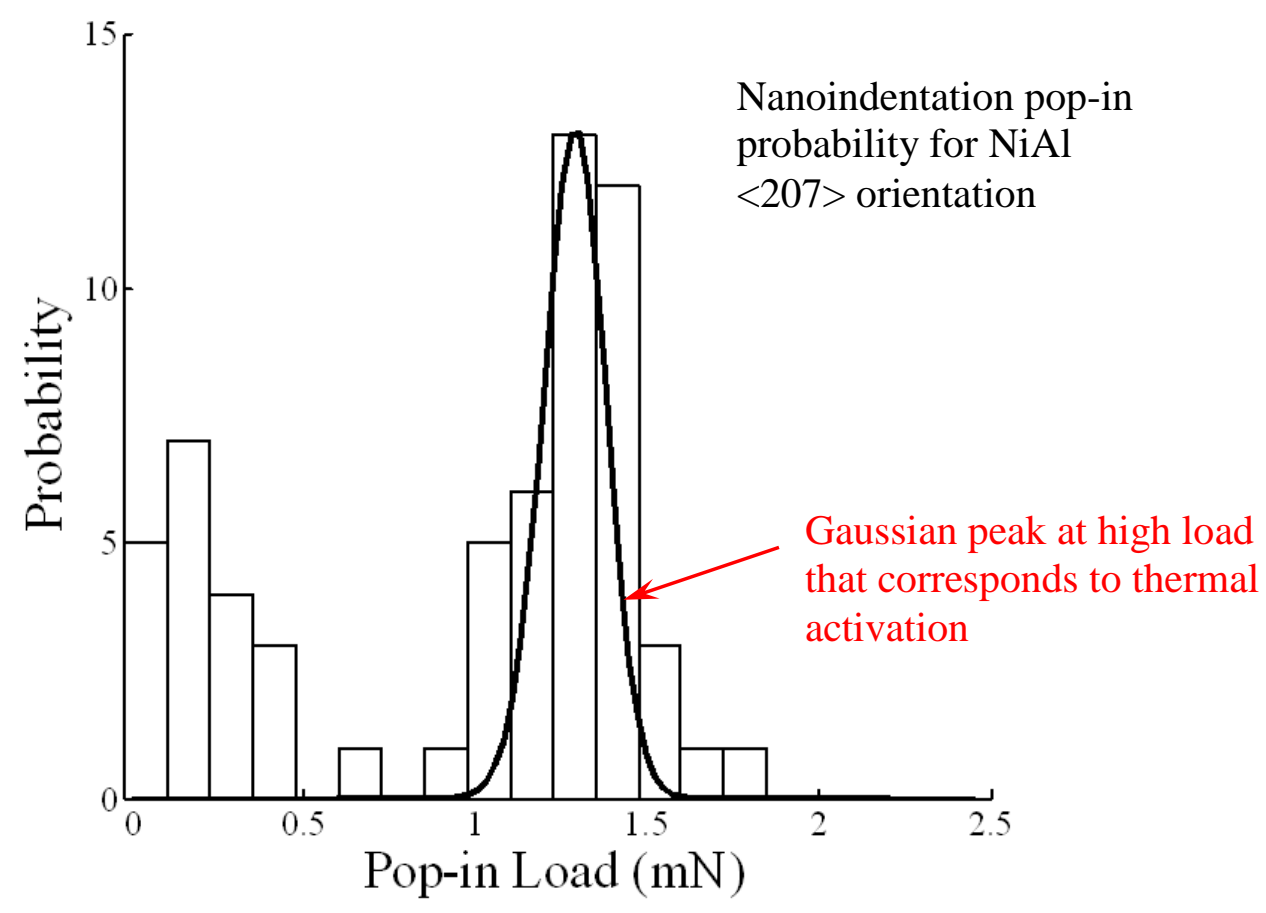

(b)

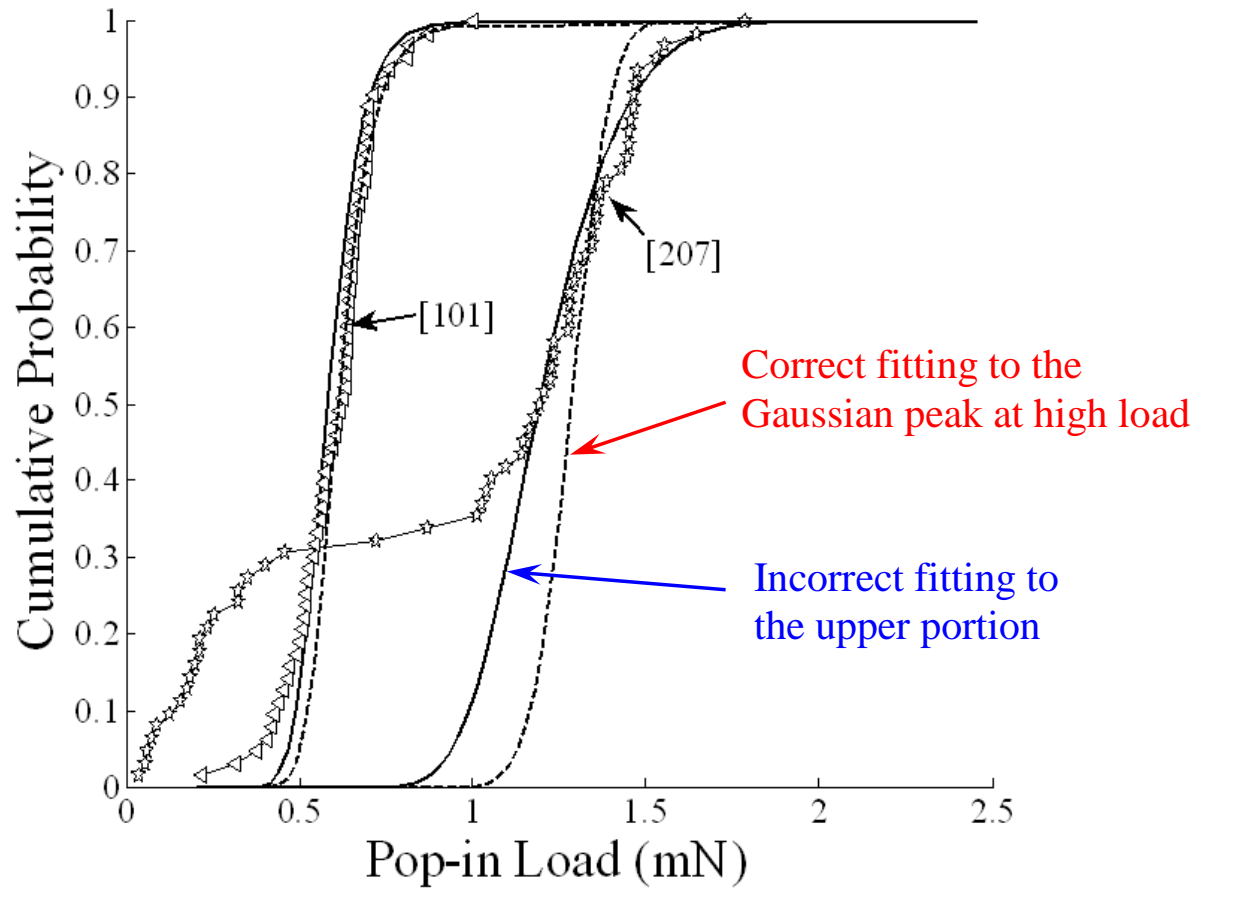

Figure 9 


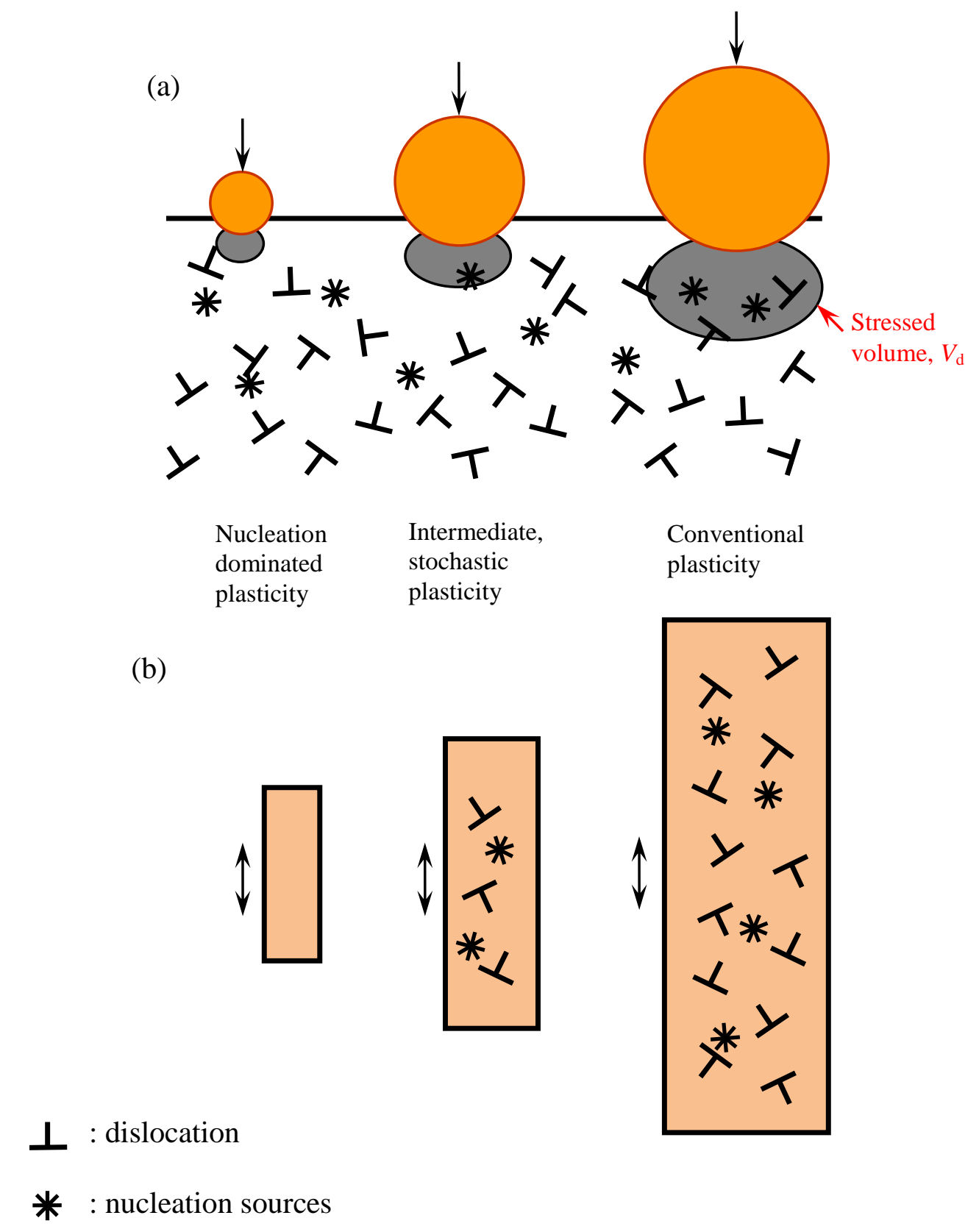

Figure 10 
(a)
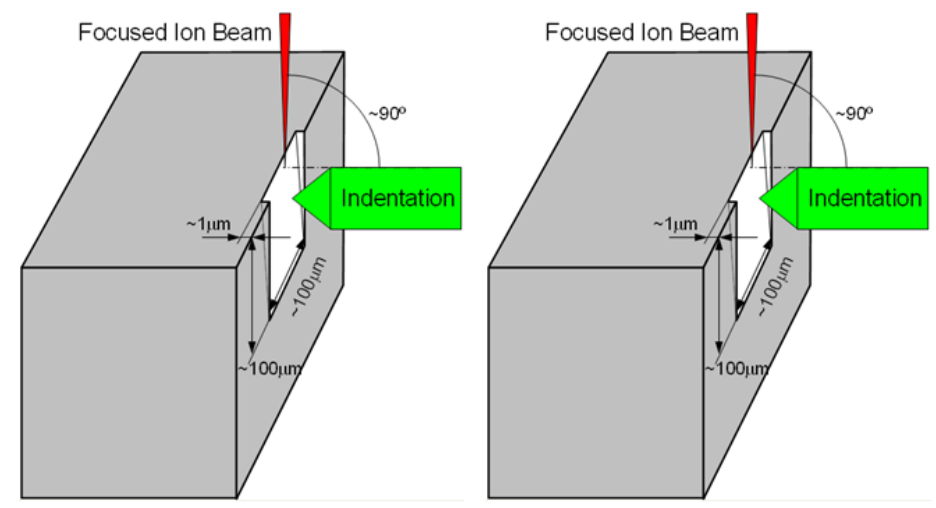

(b)

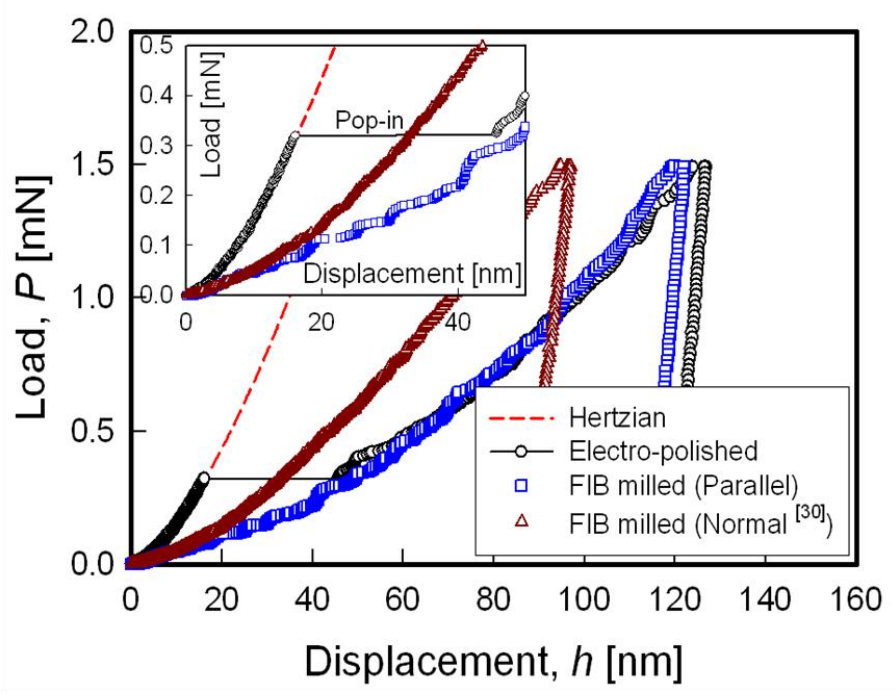

(c)

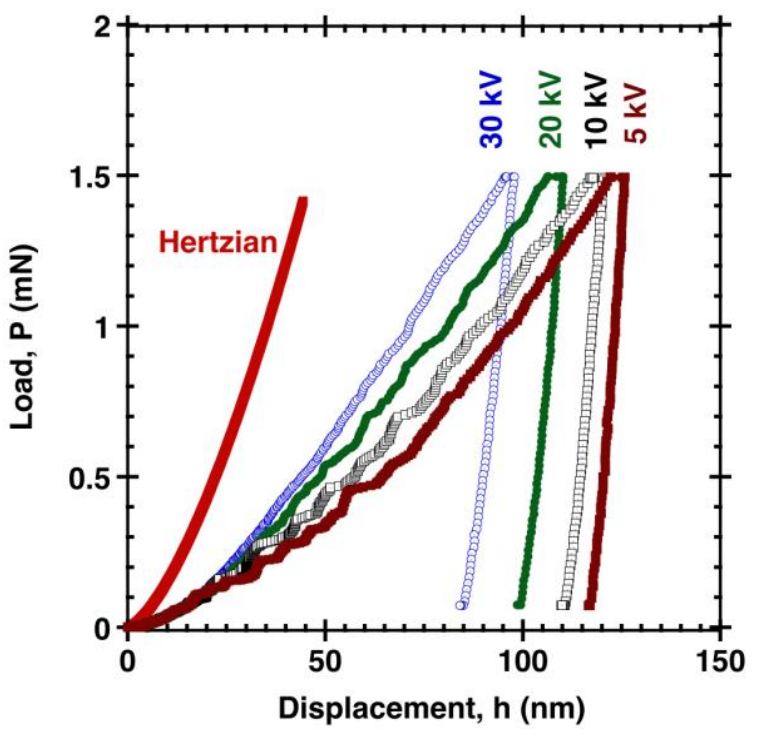

Figure 11 
(a)
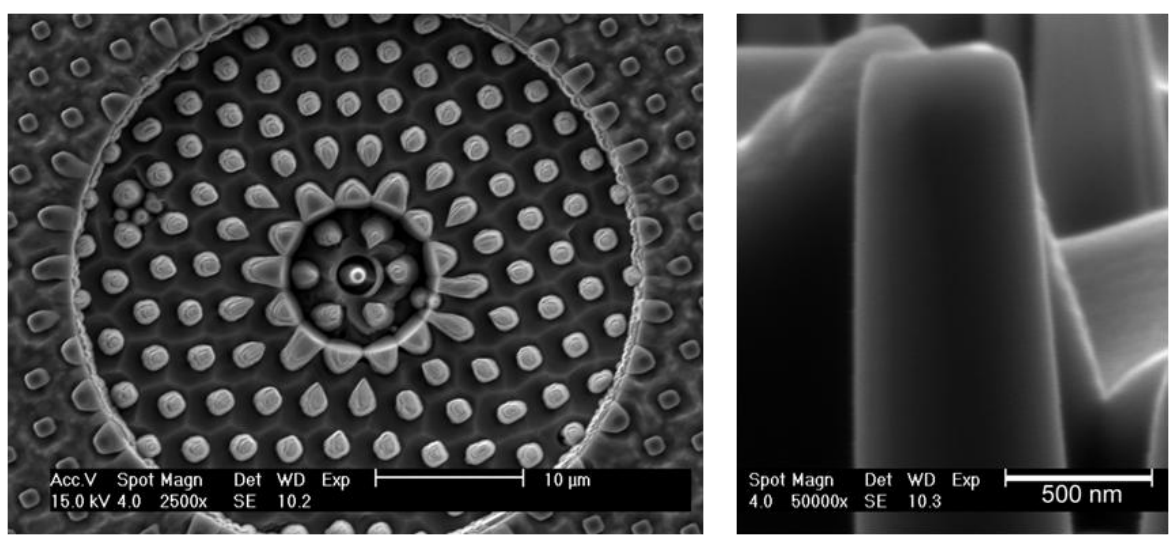

(b)

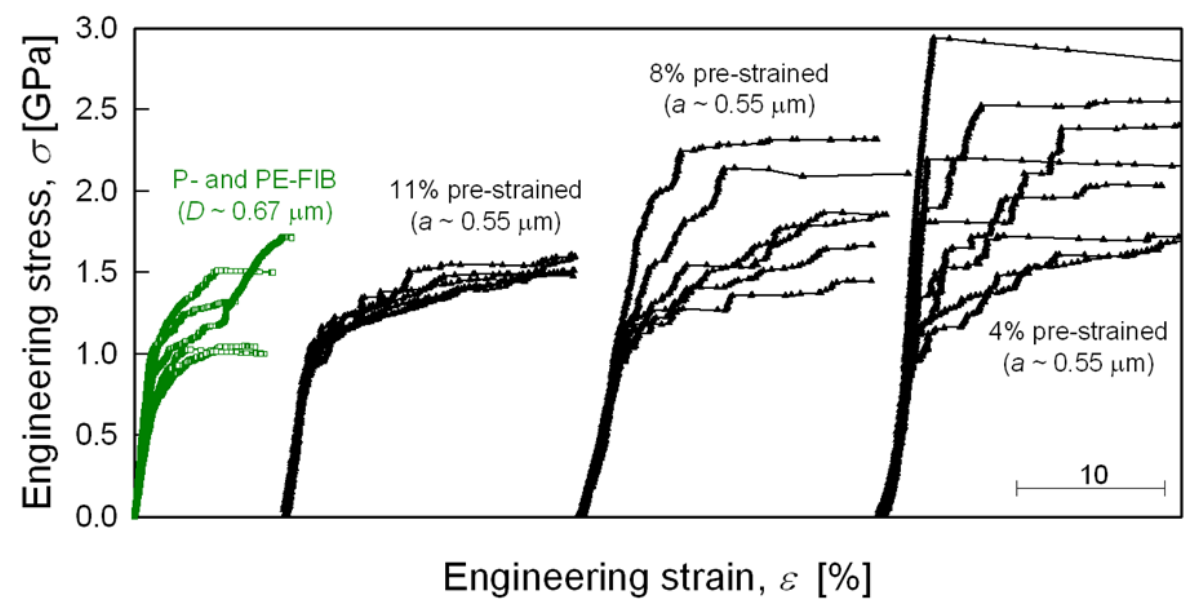

(c)

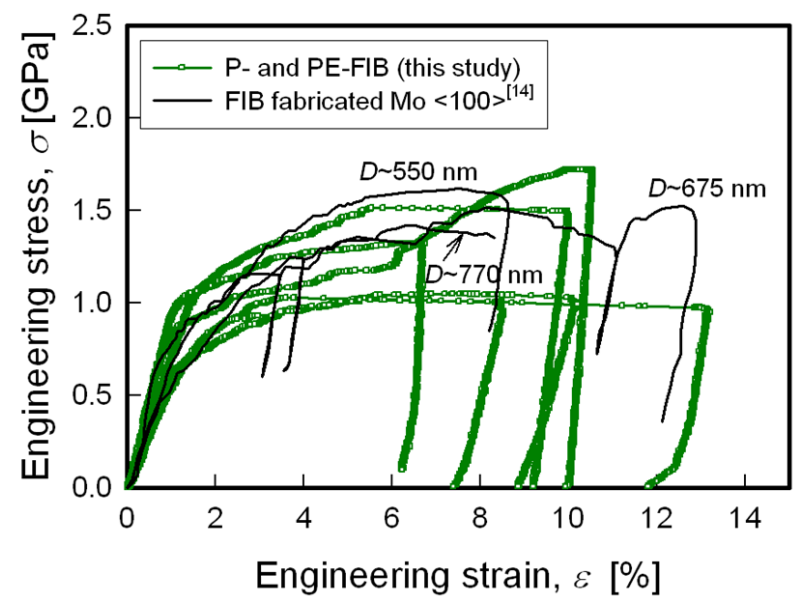

(d)
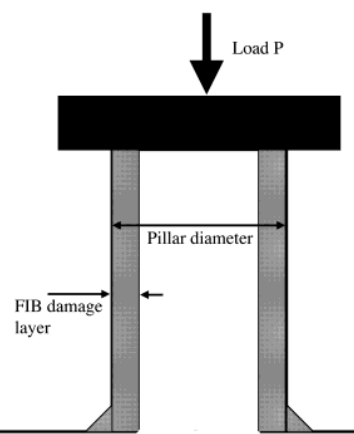

Figure 12 


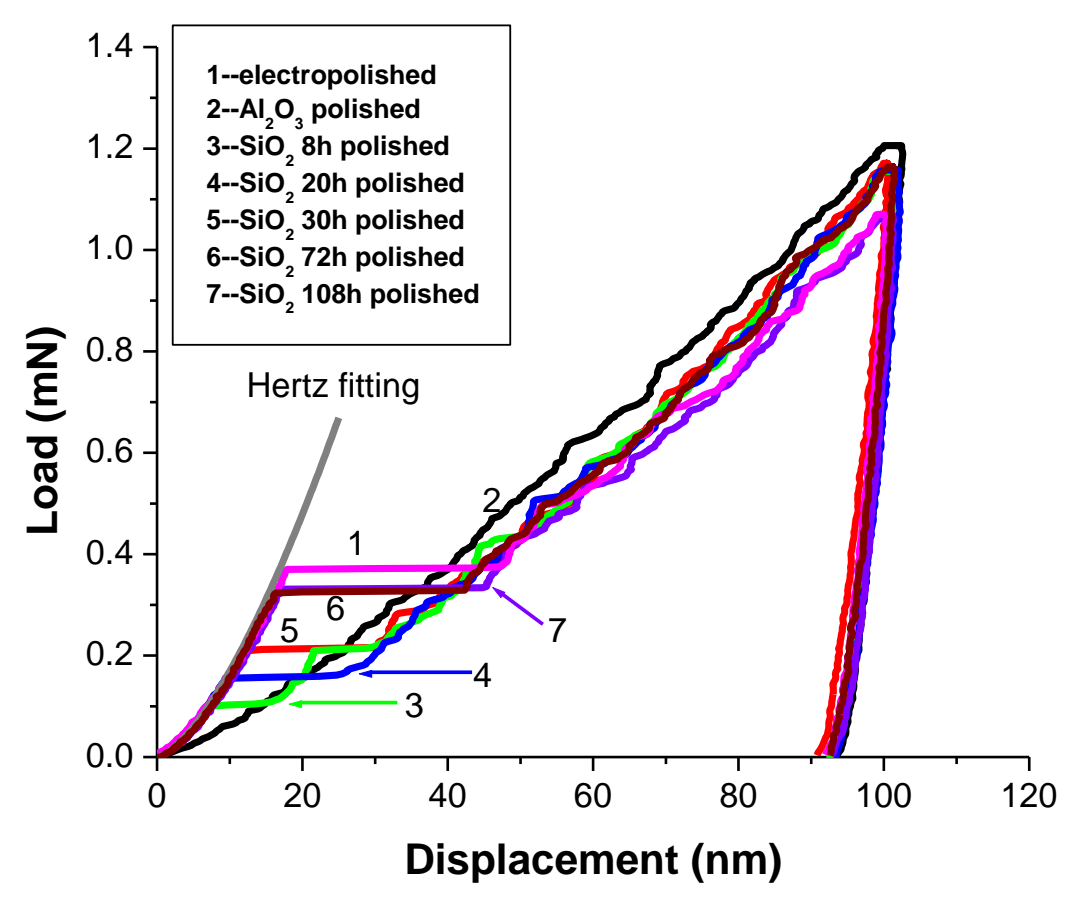

(a)

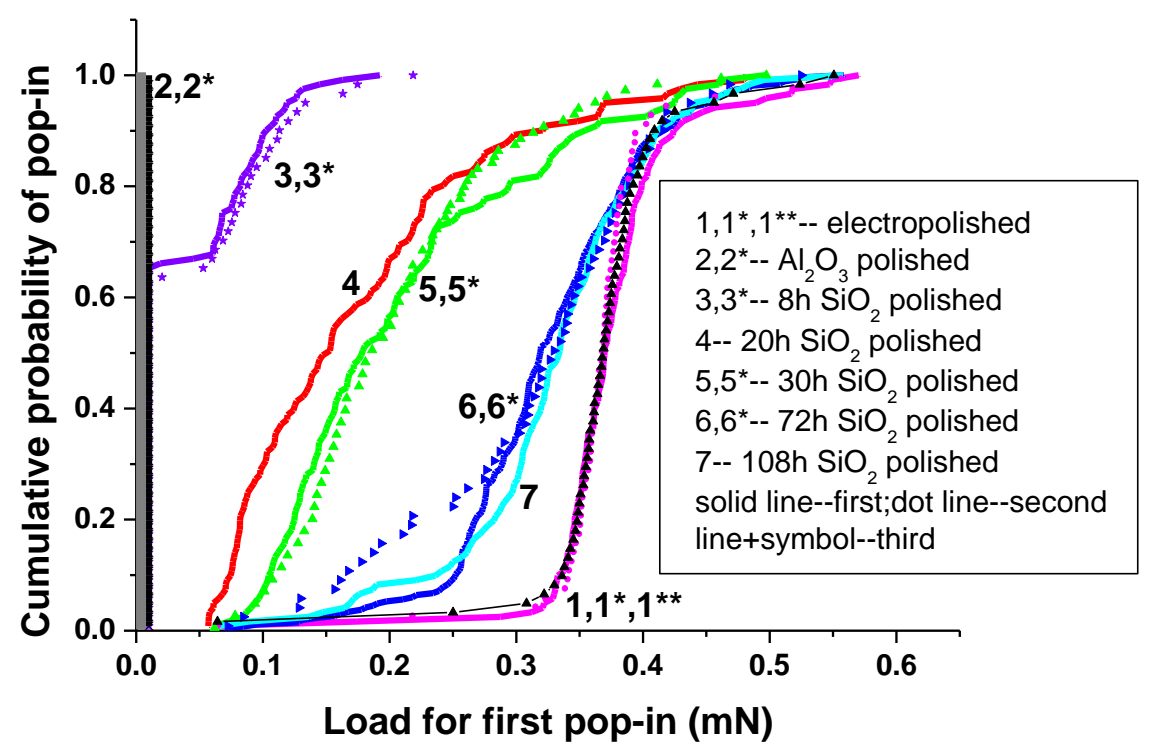

(b)

Figure 13 

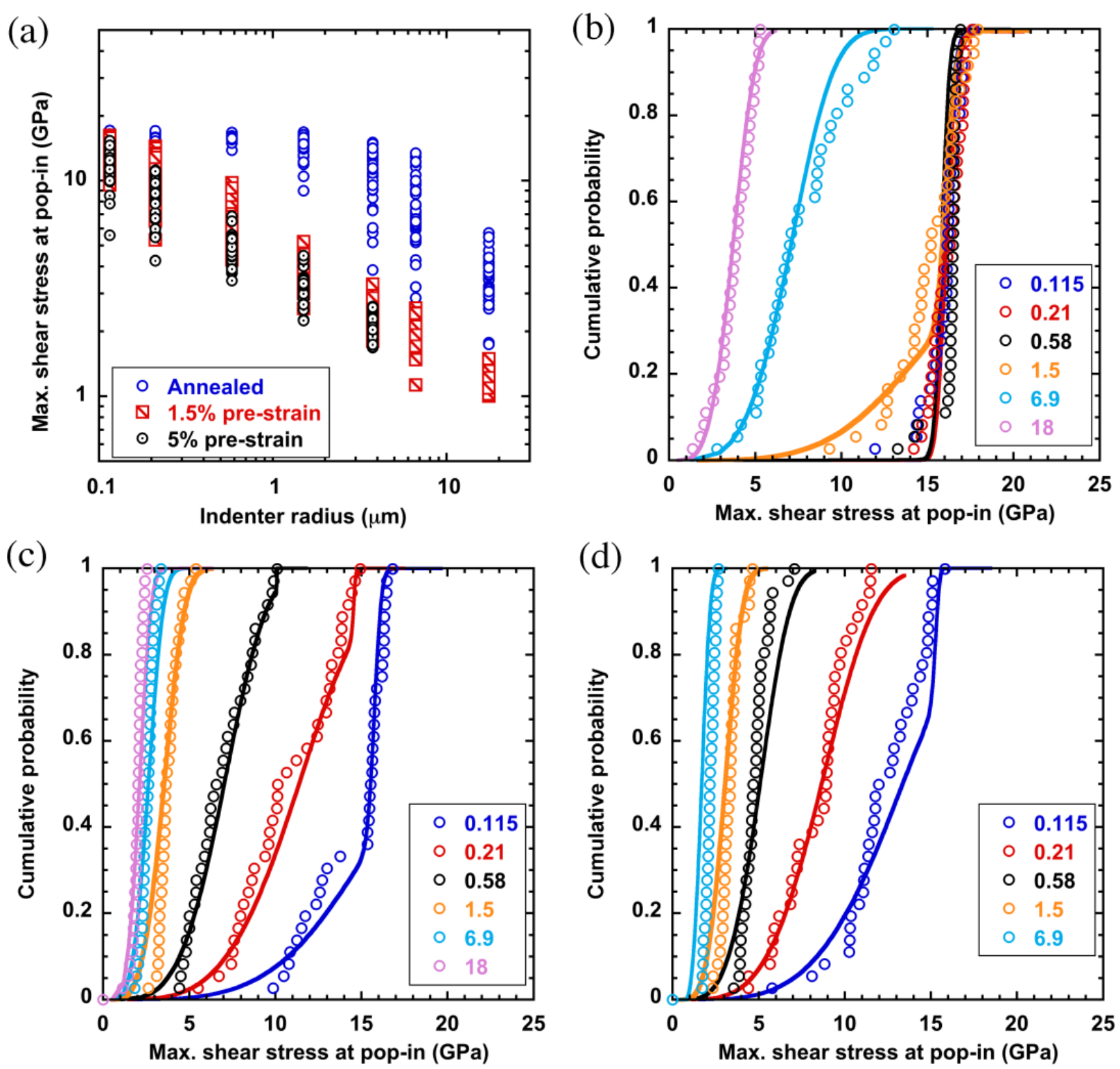

Figure 14 


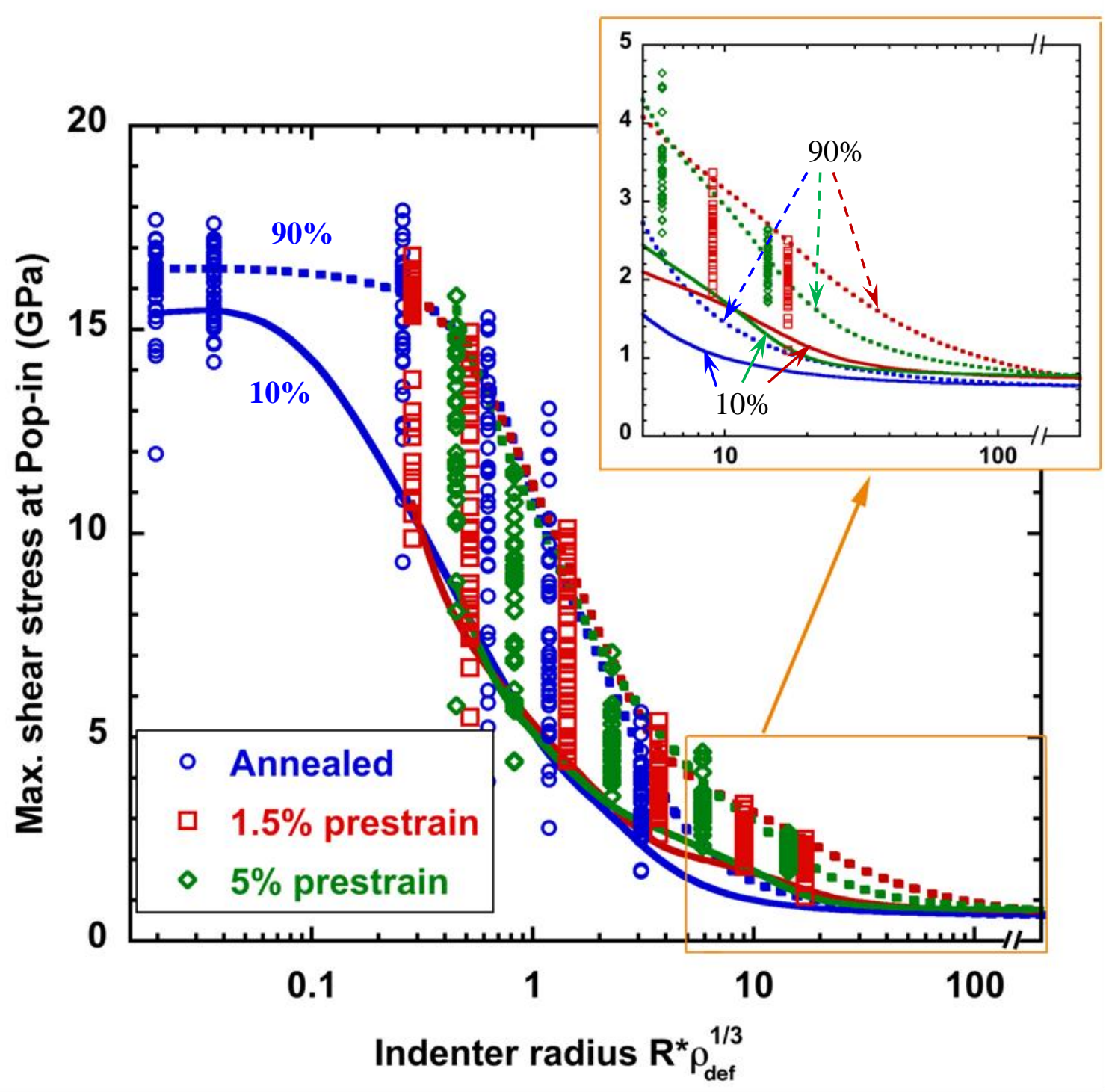

Figure 15 
(a)

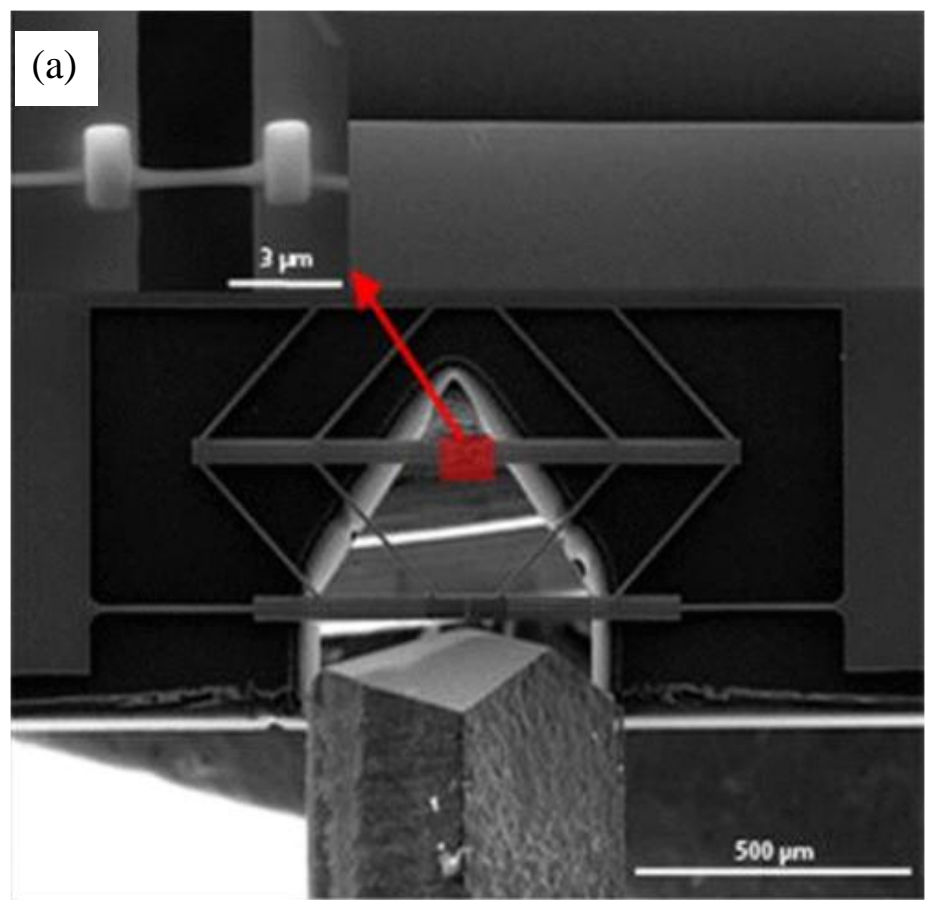

(b)

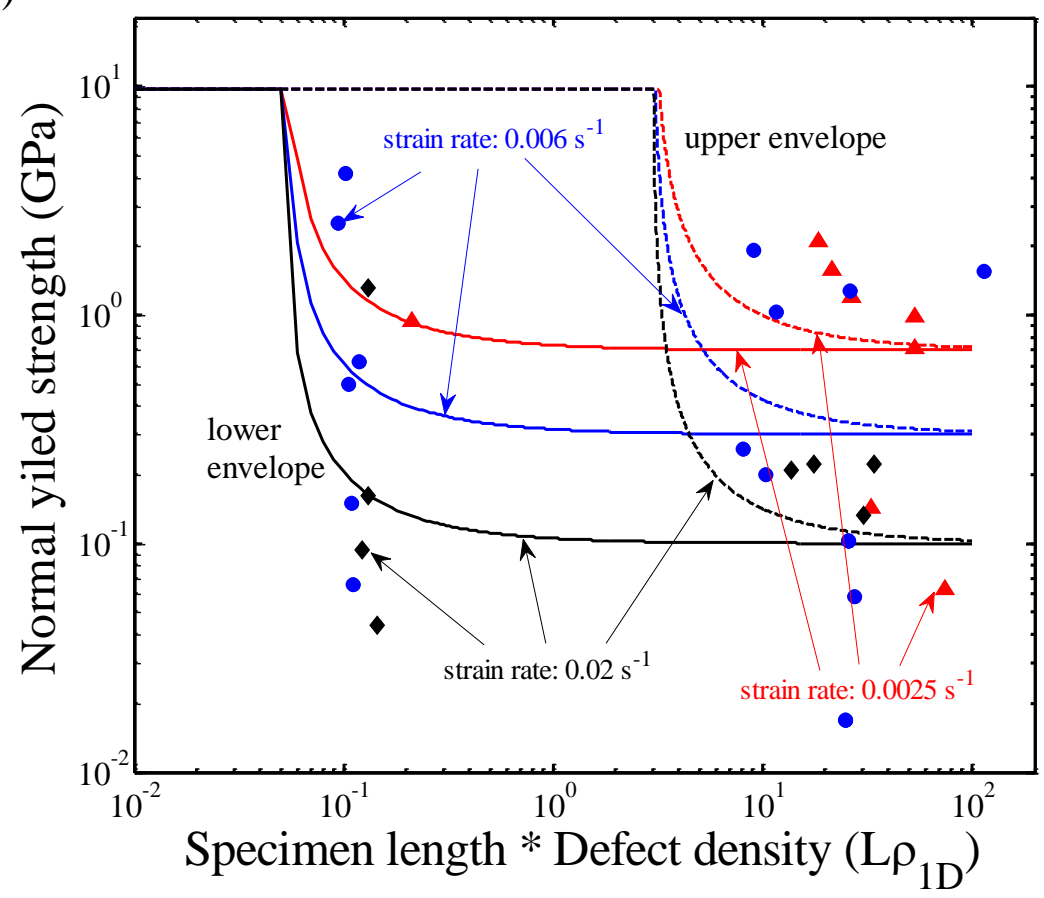

Figure 16 
(a)

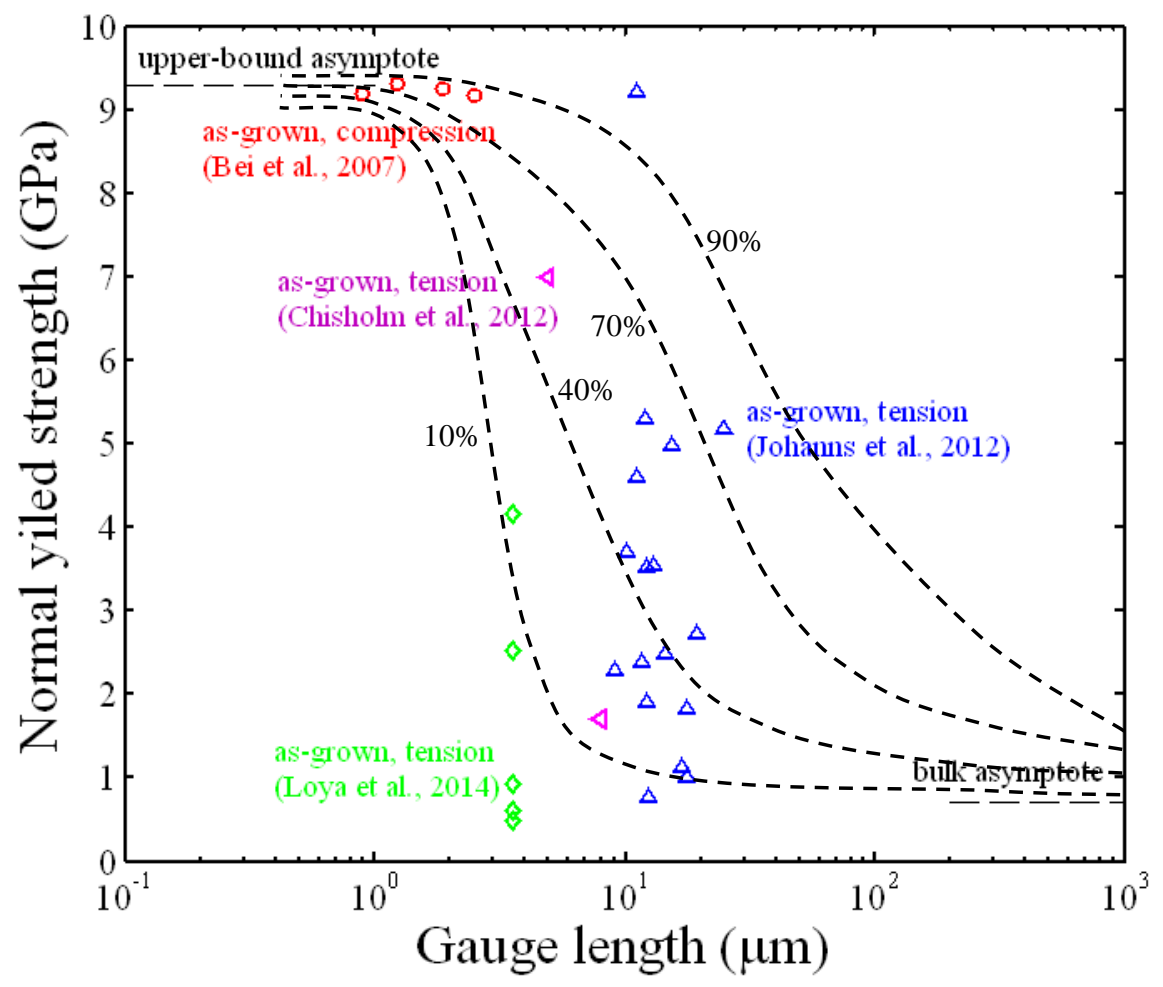

(b)

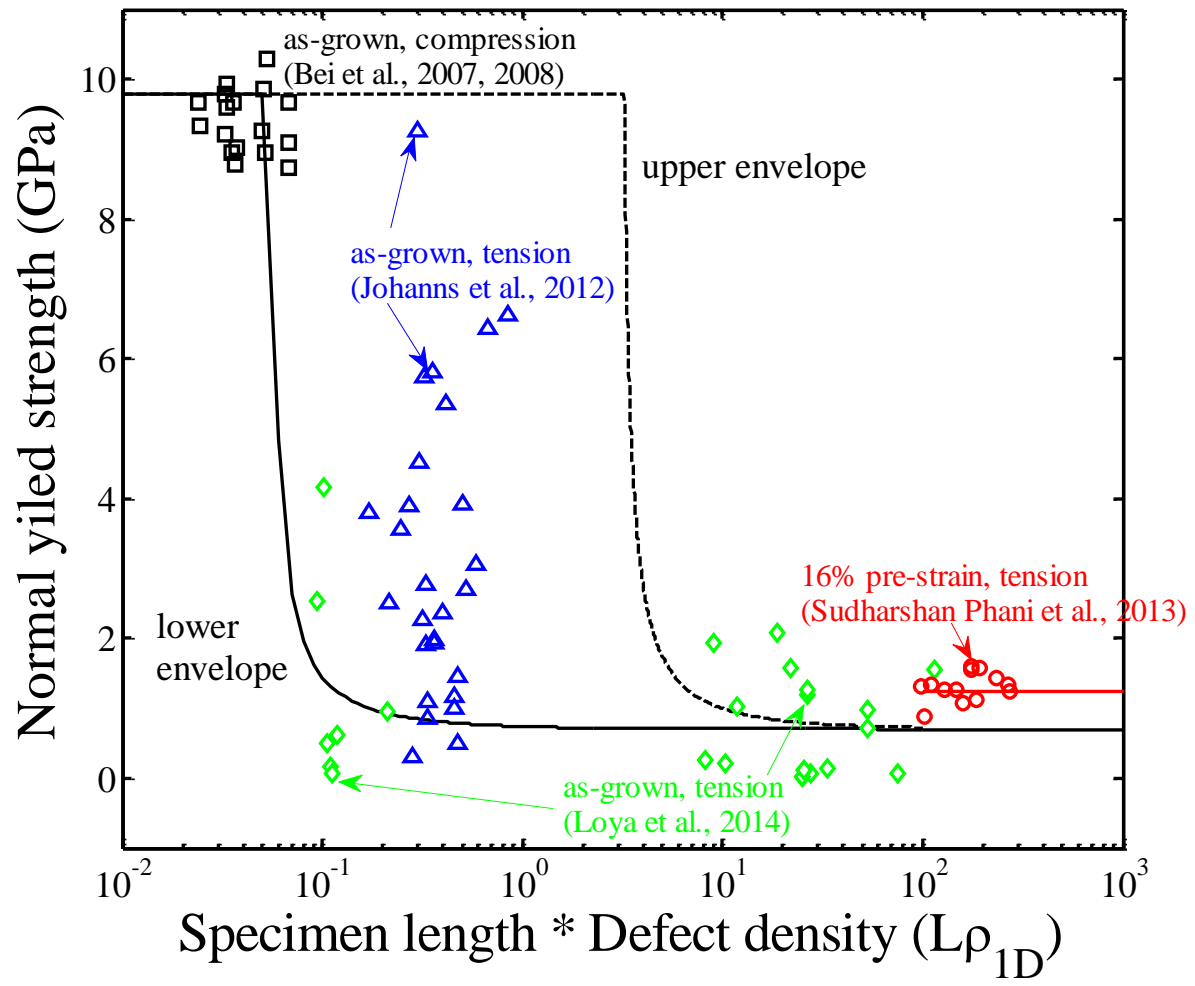

Figure 17 


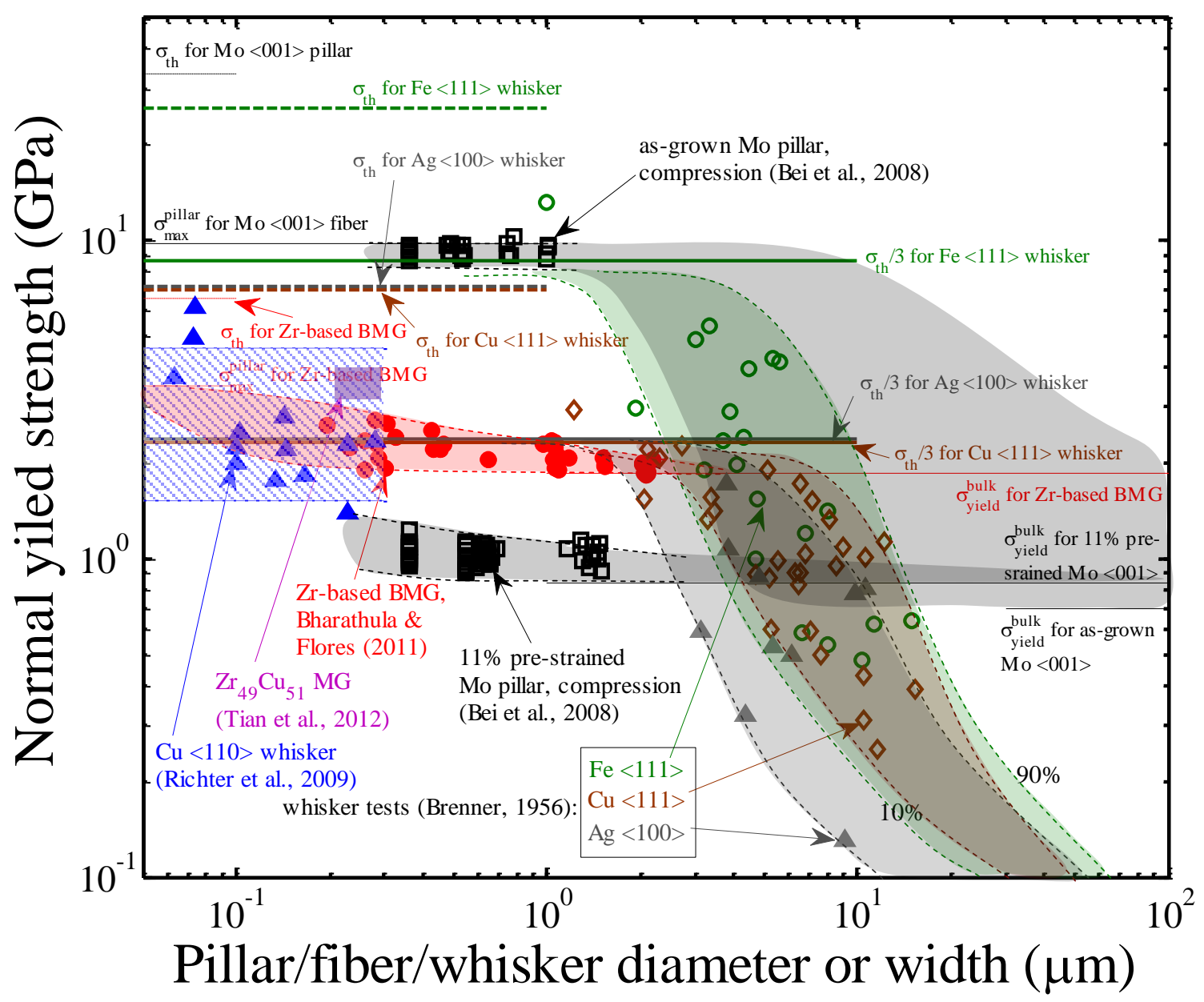

Figure 18 

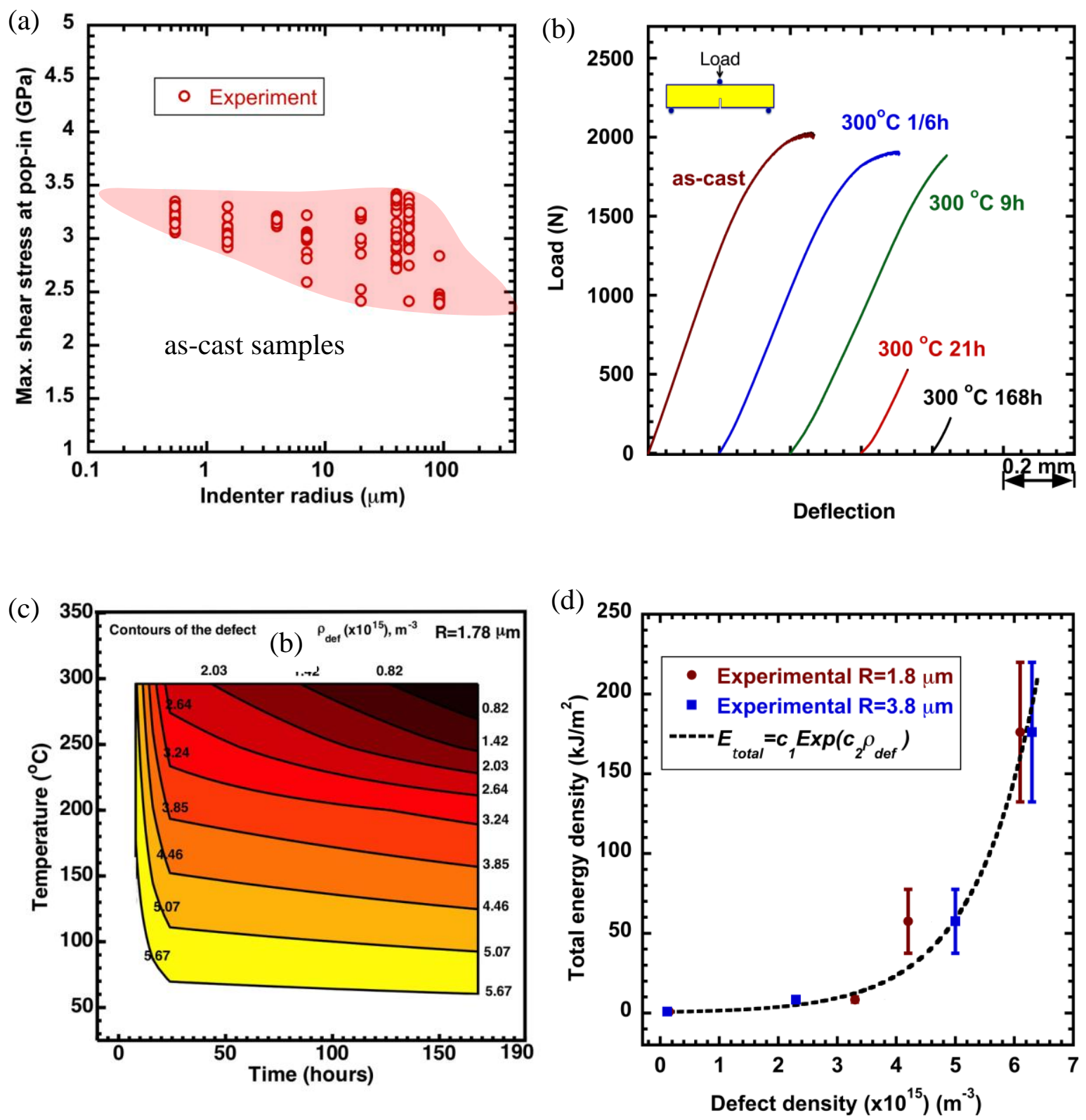

Figure 19 

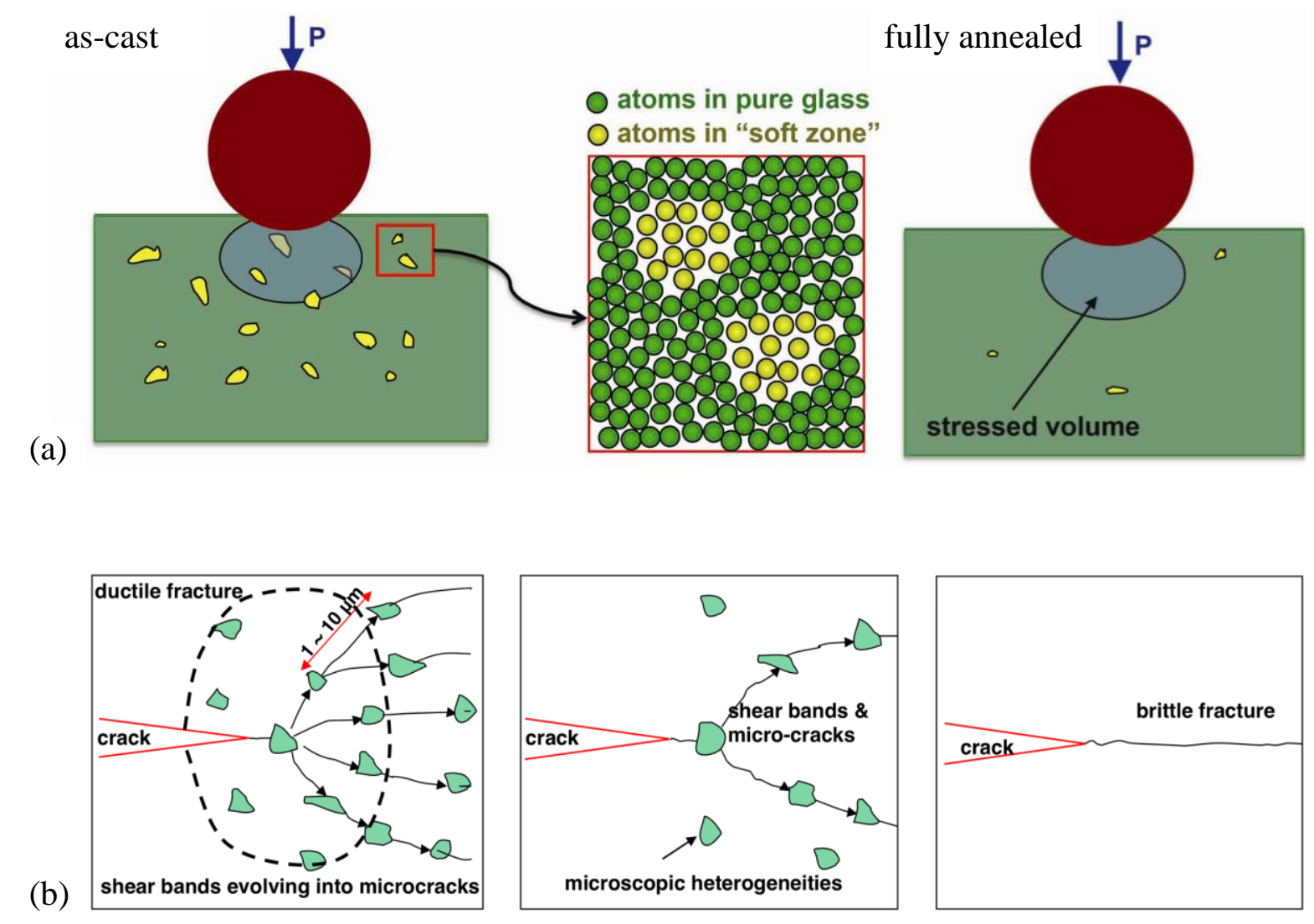

Figure 20 


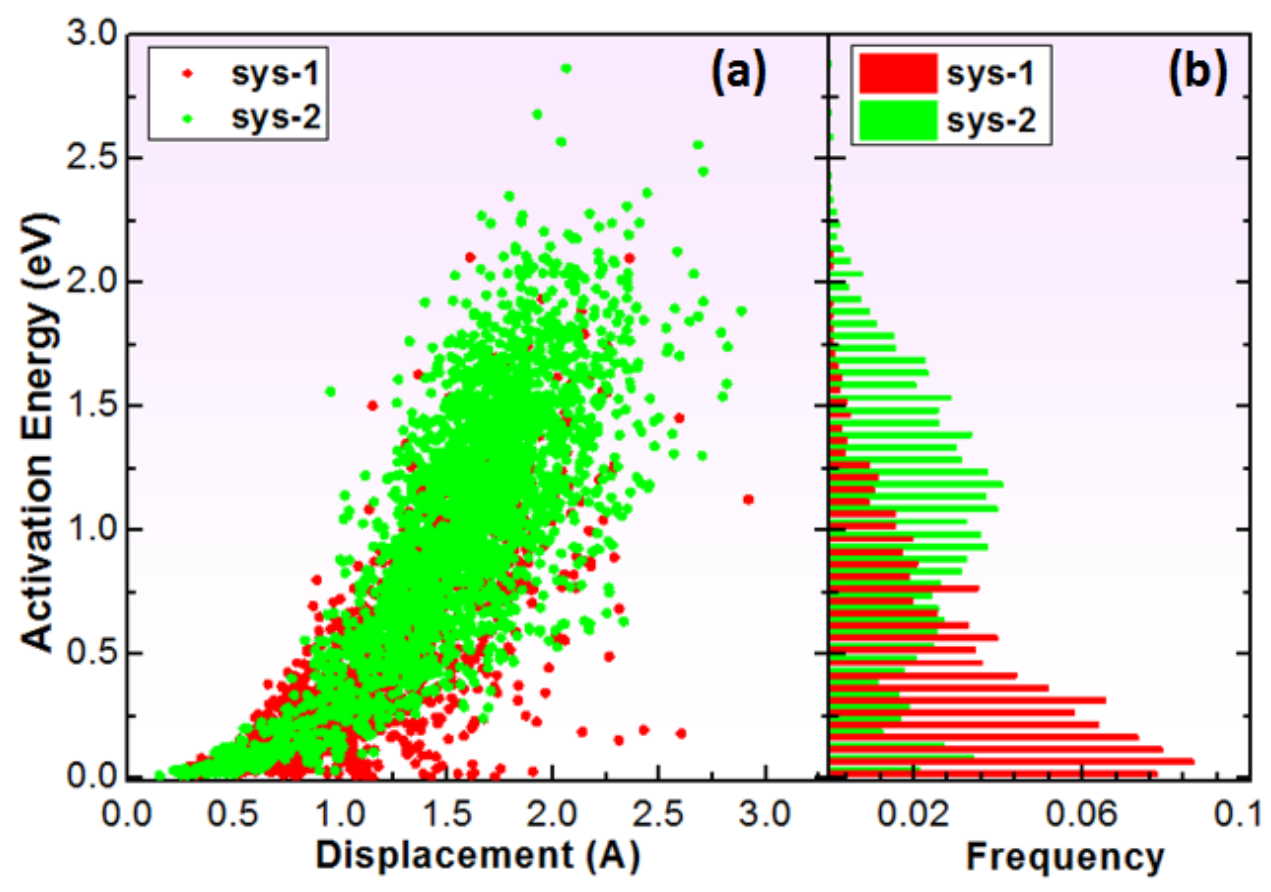

Figure 21 